\title{
Emissive Single-Crystalline Boroxine-Linked Colloidal Covalent Organic Frameworks
}

Austin Evans, loannina Castano, Alexandra Brumberg, Lucas R. Parent, Amanda Corcos, Rebecca Li, Nathan C. Flanders, David J. Gosztola, Nathan Gianneschi, Richard Schaller, William Dichtel

Submitted date: 17/08/2019 - Posted date: 19/08/2019

Licence: CC BY-NC-ND 4.0

Citation information: Evans, Austin; Castano, loannina; Brumberg, Alexandra; Parent, Lucas R.; Corcos, Amanda; Li, Rebecca; et al. (2019): Emissive Single-Crystalline Boroxine-Linked Colloidal Covalent Organic Frameworks. ChemRxiv. Preprint.

The synthesis of periodic two-dimensional (2D) polymers and characterization of their optoelectronic behaviors are challenges at the forefront of polymer chemistry and materials science. Recently, we showed that layered 2D polymers known as 2D covalent organic frameworks (COFs) can be synthesized as single crystals by preparing COF particles as colloidal suspensions. Here we expand this approach from the condensation of boronic acids and catechols to the dehydrative trimerization of polyboronic acids. The resulting boroxine-linked colloids are the next class of 2D COFs to be obtained as single-crystalline particles, as demonstrated here for four 2D COFs and one 3D COF. Colloidal stabilization enables detailed structural analysis by synchrotron X-ray diffraction and high-resolution transmission electron microscopy. Solution fluorescence spectroscopy revealed that the COF crystallites are highly emissive compared to their respective monomer solutions. Excitation-emission matrix fluorescence spectroscopy indicated that the origin of this enhanced emission can be attributed to through-space communication of chromophores between COF sheets. These observations will motivate the development of colloidal COF systems as a platform to organize functional aromatic systems into precise and predictable assemblies with emergent properties.

File list (3)

2019_08_ChemRxiv_BoroxineCOF_Manuscript.pdf (3.96 MiB) view on ChemRxiv • download file 2019_08_ChemRxiv_BoroxineCOF_SI.pdf (5.42 MiB) view on ChemRxiv - download file 


\section{Emissive Single-Crystalline Boroxine-Linked Colloidal Covalent Organic Frameworks}

Austin M. Evans, ${ }^{1}$ Ioannina Castano, ${ }^{1}$ Alexandra Brumberg, ${ }^{1}$ Lucas R. Parent,,${ }^{1,2,3}$ Amanda R. Corcos, ${ }^{1 \ddagger}$ Rebecca L. Li, ${ }^{1}$ Nathan C. Flanders, ${ }^{1}$ David J. Gosztola, ${ }^{7}$ Nathan C. Gianneschi, ${ }^{1-6}$ Richard D. Schaller, ${ }^{1,7}$ and William R. Dichtel ${ }^{1,4 *}$

${ }^{1}$ Department of Chemistry, Northwestern University, Evanston, IL 60208, USA.

${ }^{2}$ Department of Materials Science and Engineering, Northwestern University, Evanston, IL 60208, USA.

${ }^{3}$ Department of Biomedical Engineering, Northwestern University, Evanston, IL 60208, USA.

${ }^{4}$ International Institute for Nanotechnology, Northwestern University, Evanston, IL 60208, USA

${ }^{5}$ Simpson Querrey Institute, Northwestern University, Evanston, IL 60208, USA

${ }^{6}$ Chemistry of Life Processes Institute, Northwestern University, Evanston, IL 60208, USA

${ }^{7}$ Center for Nanoscale Materials, Argonne National Laboratory, Argonne, IL 60439, USA.

${ }^{\ddagger}$ Current Address: AAAS Science \& Technology Policy Fellow at U.S. Department of State, Washington, D.C.

*Email:wdichtel@northwestern.edu

ABSTRACT: The synthesis of periodic two-dimensional (2D) polymers and characterization of their optoelectronic behaviors are challenges at the forefront of polymer chemistry and materials science. Recently, we showed that layered 2D polymers known as 2D covalent organic frameworks (COFs) can be synthesized as single crystals by preparing COF particles as colloidal suspensions. Here we expand this approach from the condensation of boronic acids and catechols to the dehydrative trimerization of polyboronic acids. The resulting boroxine-linked colloids are the next class of 2D COFs to be obtained as single-crystalline particles, as demonstrated here for four 2D COFs and one 3D COF. Colloidal stabilization enables detailed structural analysis by synchrotron X-ray diffraction and high-resolution transmission electron microscopy. Solution fluorescence spectroscopy revealed that the COF crystallites are highly emissive compared to their respective monomer solutions. Excitation-emission matrix fluorescence spectroscopy indicated that the origin of this enhanced emission can be attributed to throughspace communication of chromophores between COF sheets. These observations will motivate the development of colloidal COF systems as a platform to organize functional aromatic systems into precise and predictable assemblies with emergent properties.

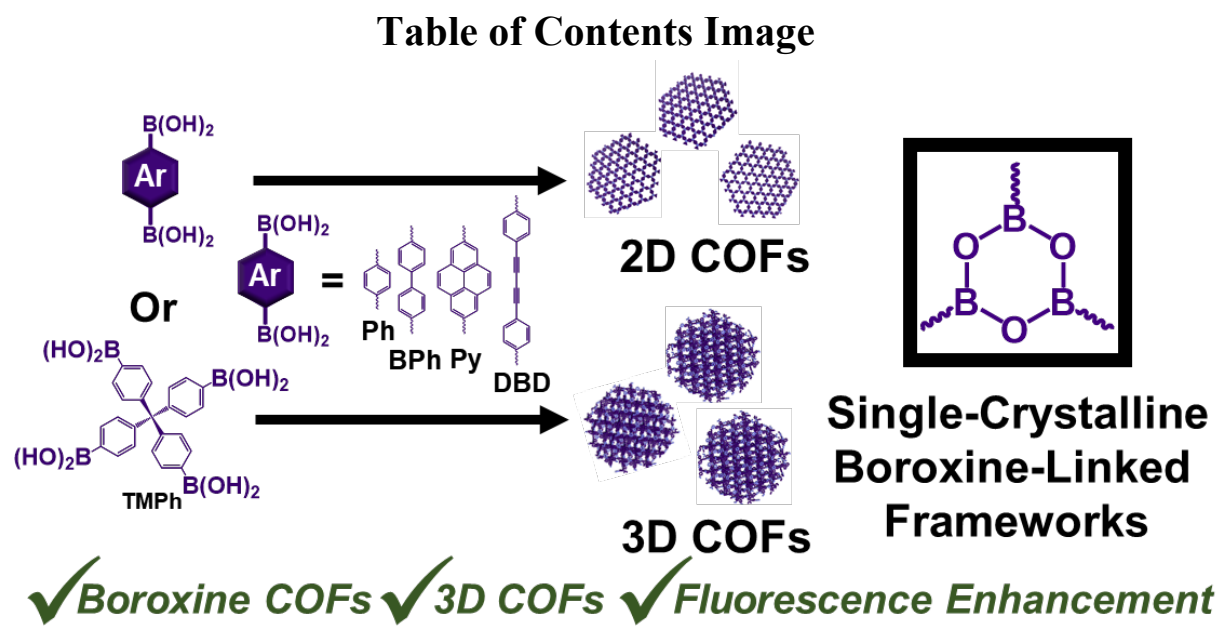


INTRODUCTION: The structural and functional diversity of layered, two-dimensional (2D) materials provides them with a collection of technologically useful properties. ${ }^{1-2}$ However, synthetically engineering $2 \mathrm{D}$ organic materials through polymerization with a desired combination of chemical functionality, porosity, topology, and electronic structure remains a challenge at the frontier of materials chemistry. ${ }^{3-5} 2 \mathrm{D}$ covalent organic frameworks (COFs) are a class of stable, ${ }^{6}$ layered, permanently porous polymers formed from the simultaneous polymerization and crystallization of directional subunits. 2D COFs are synthetically versatile materials, with several hundred reported structures to date, ${ }^{7-8}$ which have inspired interest in applications such as gas separations, catalysis, environmental remediation, energy storage, and optoelectronic devices. ${ }^{7,9-14}$ A limitation of 2D COFs is that they are typically isolated as polycrystalline powders, which complicates their structural, electronic, and optical characterization. ${ }^{15-18}$ Recently, we developed a overcame this limitation by stabilizing boronate ester-linked COFs as colloidal suspensions by including nitrile-containing cosolvents in the reaction mixture (Figure 1A). ${ }^{19-20} \mathrm{We}$ attributed this stabilization to the interaction of nitriles with boron-containing functional groups in the framework. ${ }^{20}$ This hypothesis led us to postulate that other classes of boron-linked COFs might be stabilized in a similar way.

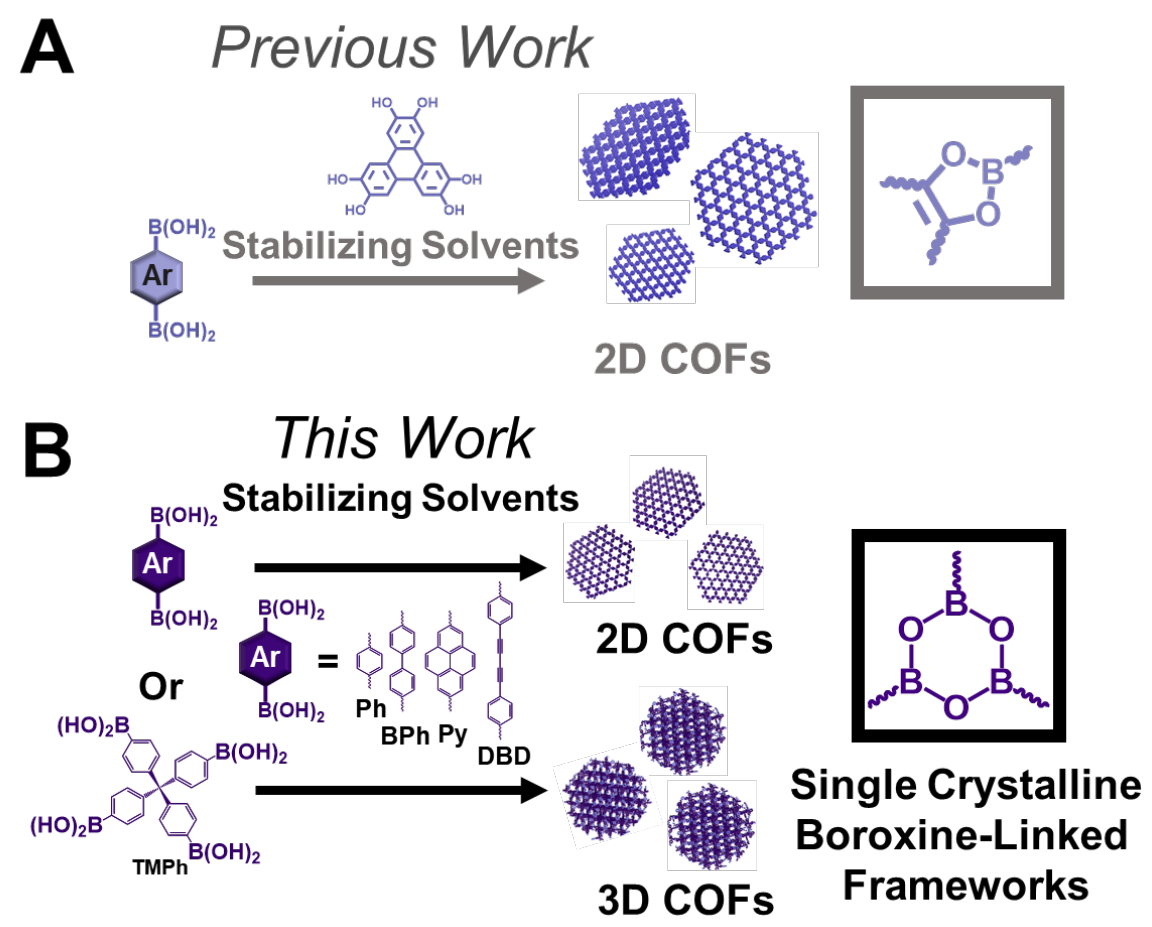

Figure 1. A) Previous work demonstrating stabilization of 2D boronate-ester COFs as colloidal suspensions. B) Colloidal stabilization is now generalized for $2 \mathrm{D}$ and $3 \mathrm{D}$ boroxine-linked COFs.

Here we demonstrate that the self-condensation of polyboronic acids in the presence of electron-donating solvents produces colloidal boroxine-linked COFs. These boroxine-linked materials are the second class of 2D COFs to be prepared as stable single-crystalline particles and more than doubles the number of 
covalent frameworks synthesized as single crystals (Figure 1B). Furthermore, we show that this approach is amenable to the synthesis of a 3D framework, further extending the generality of this controlled polymerization method.

The well-dispersed and isolable nature of these COF particles enabled their structural analysis using low-dose high-resolution transmission electron microscopy (HR-TEM) and synchrotron X-ray diffraction (XRD). By combining these techniques, we were able to gain insight into the interlayer arrangement of 2D polymer sheets, which is challenging to assign in powder COF systems but strongly affects their electronic structure. ${ }^{21-22}$ While performing these investigations, we noted that COF samples were highly fluorescent compared to their respective monomers. Therefore, excitation-emission matrix fluorescence spectroscopy (EEMS) was used to probe the intrinsic optical properties of these dispersed crystallites. EEMS revealed that the enhanced emission of the COFs originates from the formation of exciplexes as a consequence of the fluorophores' 2D layered structure. We anticipate that these results will greatly impact approaches to COF synthesis and inform the understanding of through-space electronic communication in $2 \mathrm{D}$ polymers and other framework materials.

Results and Discussion: We synthesized Ph-COF (previously reported ${ }^{23}$ as COF-1) in mixtures of $\mathrm{CH}_{3} \mathrm{CN}$ and 4:1 1,4-dioxane:mesitylene. The 1,4-phenylenebis(boronic acid) (PBBA) monomer was first dissolved in the specified solvent mixture at a concentration of $10 \mathrm{mM}$ and then the container was sealed, sonicated, and heated to $90{ }^{\circ} \mathrm{C}$ for an additional 3 days (Figure $\mathbf{2 A}$ ). When $0-30$ vol\% $\mathrm{CH}_{3} \mathrm{CN}$ was included in the reaction mixture, a white precipitate formed over the course of 3 days. These precipitates were isolated and characterized via Fourier-transform infrared spectroscopy (FT-IR) and XRD, the results of which were consistent with previous reports of Ph-COF (Figure S11). ${ }^{23}$ In contrast, no precipitation was observed when $30-80$ vol\% of $\mathrm{CH}_{3} \mathrm{CN}$ was included in the reaction mixture while over the course of 3 days these solutions changed from transparent to opaque-white suspensions (Figure 2B) that were stable for at least 6 months. When $>80 \mathrm{vol} \%$ of $\mathrm{CH}_{3} \mathrm{CN}$ was included in the reaction mixture, the heated monomer solution never became transparent, which we attribute to the insufficient solubility of PBBA under these conditions. These observations indicate that boroxine polymerization is not inhibited by the presence of $\mathrm{CH}_{3} \mathrm{CN}$ and that a specific range of solvent mixtures is necessary for the successful solubilization of PBBA and stabilization of the Ph-COF particles. 


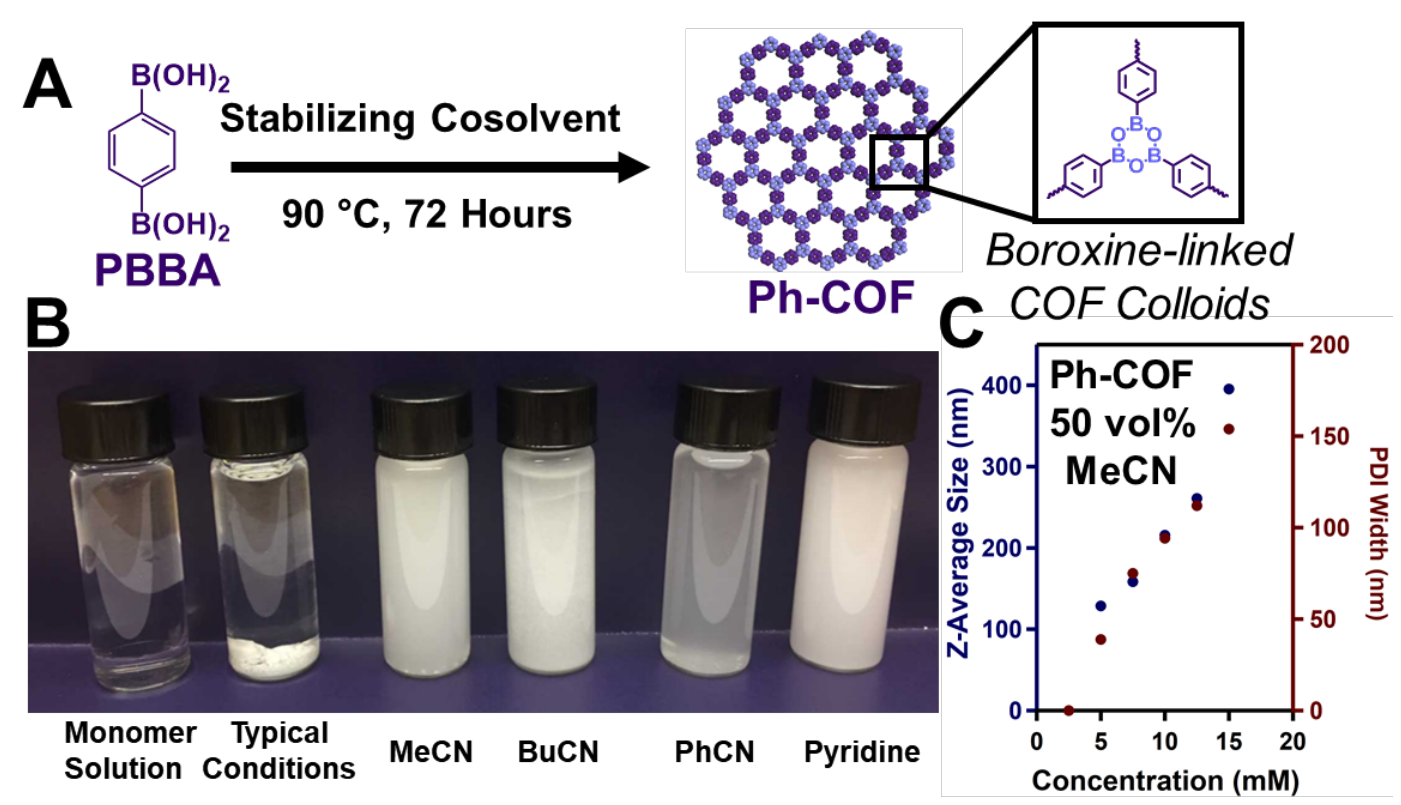

Figure 2. A) Synthesis of boroxine-linked Ph-COF particles through solvothermal condensation of PBBA $(10 \mathrm{mM})$. B) Photograph comparing the transparent monomer solution to typically isolated $\mathbf{P h}-\mathbf{C O F}$ powders ${ }^{23}$ and dispersed Ph-COF crystallites synthesized in the presence of electron-donating solvents. C) Dynamic light scattering (DLS) Z-average size and polydispersity index (PDI) of Ph-COF synthesized with variable initial PBBA concentrations.

Inclusion of other nitrile cosolvents (butyronitrile [BuCN], propionitrile [PrCN], and benzonitrile $[\mathrm{PhCN}])$ at $50 \mathrm{vol} \%$ of the solvent mixture stabilized $\mathbf{P h}-\mathbf{C O F}$ particles in a similar fashion to $\mathrm{CH}_{3} \mathrm{CN}$, in which transparent solutions became cloudy over the course of heating for 3 days (Figure 2B). The inclusion of pyridine in the reaction mixture also resulted in a colloidal suspension of Ph-COF particles. However, the transition to a cloudy suspension occurred within approximately 1 minute (Supplementary Video 1), which may be due to its stronger interaction with boronic acids and boroxines because of its stronger basicity. ${ }^{24}$ These results show that the inclusion of Lewis-basic cosolvents in boroxine-linked COF syntheses results in stable colloidal suspensions. These observations are consistent with previous reports that described Lewis-basic cosolvents coordinated to the Lewis-acidic sites of boron-linked COFs. ${ }^{25-27}$ Taken together, stabilization by electron-donating solvents suggests a direct interaction between the cosolvent and COF surface, which we speculate is responsible for the observed colloidal stability, as has been found for other classes of nanoparticles. ${ }^{28}$

A central advantage of stable nanocrystal suspensions is that their size can be controlled by varying the synthesis and growth conditions. ${ }^{19} \mathbf{P h}-\mathbf{C O F}$ particle sizes were controlled by varying the initial PBBA concentration. We found that concentrations lower than $5 \mathrm{mM}$ did not produce colloidal particles, presumably because this is below the critical nucleation concentration for these conditions. ${ }^{29}$ In contrast, concentrations above $15 \mathrm{mM}$ were not initially soluble and produced a mixture of $\mathbf{P h}$-COF nanoparticles and powder precipitate. However, within the regime where Ph-COF nanoparticles were formed exclusively (5-15 mM PBBA), dynamic light scattering (DLS) measurements indicate that initial 
monomer concentration is positively correlated with the Z-average size, with particle sizes ranging between 100 and $400 \mathrm{~nm}$, and a polydispersity index (PDI) width between 50 and $200 \mathrm{~nm}$ (Figure 2C). ${ }^{30}$ This behavior is consistent with linear condensation polymerizations in which the starting monomer concentration leads to larger average chain length and dispersity. ${ }^{31}$ This observation highlights the potential of using colloidal nanocrystals as a platform to explore mechanisms of COF nucleation and elongation. ${ }^{18-19,29}$
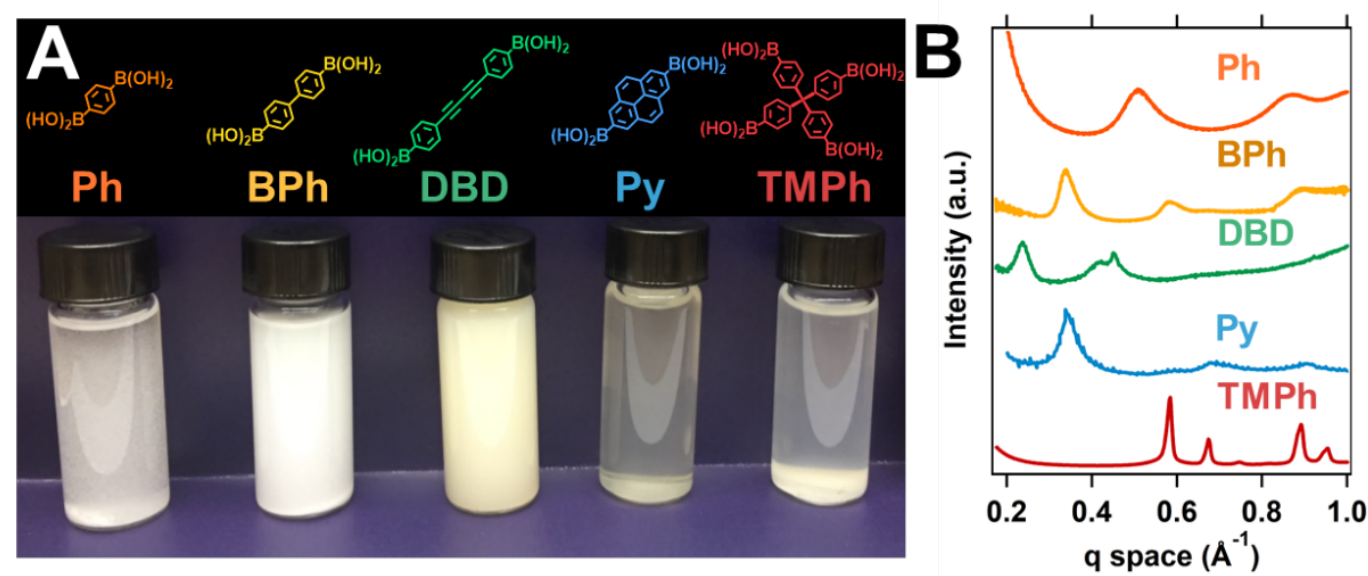

Figure 3. A) Monomer structures and optical images of structurally diverse COF nanoparticle suspensions. B) X-ray diffraction (grazing-incidence of dropcast crystallites: TMPh-COF or in solvo wide-angle X-ray scattering: Ph-COF, BPh-COF, DBD-COF, Py-COF) of boroxine-linked COF nanocrystals.

A defining feature of COF chemistry is the modular approach used to synthesize isoreticular crystalline, porous, organic polymers. Interested in understanding the synthetic versatility of boroxine-linked COF colloid stabilization, we self-condensed several polyfunctional boronic acids to form their corresponding boroxine-linked COFs, including 2,7-pyrenebisboronic acid (Py-COF), tetraphenylmethylboronic acid (TMPh-COF), and two previously unreported COF structures condensed from, 4,4'-biphenylbisboronic acid (BPh-COF) and diphenylbutadiynebisboronic acid (DBD-COF). All self-condensations were performed in a solvent mixture of $\mathrm{CH}_{3} \mathrm{CN}: 1$,4-dioxane:mesitylene 50:40:10 vol\% by heating the respective monomers of each $\mathrm{COF}$ at $90{ }^{\circ} \mathrm{C}$ for 3 days. The solutions became cloudy for all reactions investigated, indicating the formation of solution-stabilized nanoparticles (Figure 3A). ${ }^{32}$

Synchrotron X-ray diffraction of the colloidal boroxine-linked COF suspensions confirmed their crystallinity and identity (Figure 3B). Diffraction features were observed for Ph-COF at $0.55 \AA^{-1}$ and $0.85 \AA^{-1}$, corresponding to the (100) and (110) Bragg features, respectively. The location of these features is consistent with those reported by Côte et al., which were assigned to an AB offset structure of $\mathbf{P h}-\mathbf{C O F}$ in which adjacent layers are offset by half a unit cell (Figure S23). ${ }^{23}$ However, the relative intensities of these features were inconsistent with this report. Due to the solvated nature of these crystallites, it was challenging for us to definitively assign their interlayer arrangement based on the few, weak diffraction 
signals observed here. The (100) diffraction feature for both the known Py-COF and previously unexplored BPh-COF occur at $0.39 \AA^{-1}$, consistent with the nearly equivalent lattice dimensions of these two COFs (Figure S24 and S26). This feature, along with higher order diffractions, allowed us to assign these materials as having AA eclipsed structures. The location of the primary diffraction feature ((200) at $0.21 \AA^{-1}$ ) for the newly synthesized DBD-COF was consistent with the hexagonal unit cell size expected for a linker of this length. However, its relative intensity pattern was unusual when compared to those of other hexagonally symmetric COFs. ${ }^{33}$ Ultimately, we attributed this diffraction pattern to a partially offset structure. We hypothesize that this stacking is governed by the interaction of stacked boroxines given the relatively weak van der Waals forces associated with the COF diphenylbutadiyne moieties. ${ }^{34}$ Finally, the 3D boroxine-linked COF, TMPh-COF, produced diffraction features at 0.59, 0.62, 0.91, and $0.96 \AA^{-1}$, which correspond to the (211), (220), (310), and (321) features, respectively. This diffraction pattern is consistent with the previously reported non-interpenetrated diamondoid structure for this material. ${ }^{35}$ Together, these results show that colloidally stable particles exhibit the defining structural regularity of COF materials. Additionally, these diffraction results demonstrate that chemically and topologically diverse framework materials are amenable to colloidal stabilization. The limited number and strength of the diffraction features for 2D COFs, including those reported here, impedes definitive assignments of stacking arrangements. ${ }^{10}$ Secondary structural characterization of agglomerated polycrystalline COF powders is also challenging and has limited the number of reports aimed at in-depth structural characterization using direct imaging techniques, such as electron microscopy. ${ }^{36}$ In contrast, dispersed crystallites are an ideal morphology for microscopic structural characterization. With this in mind, we set out to explore COF nanocrystals via high-resolution transmission electron microscopy (HR-TEM). 


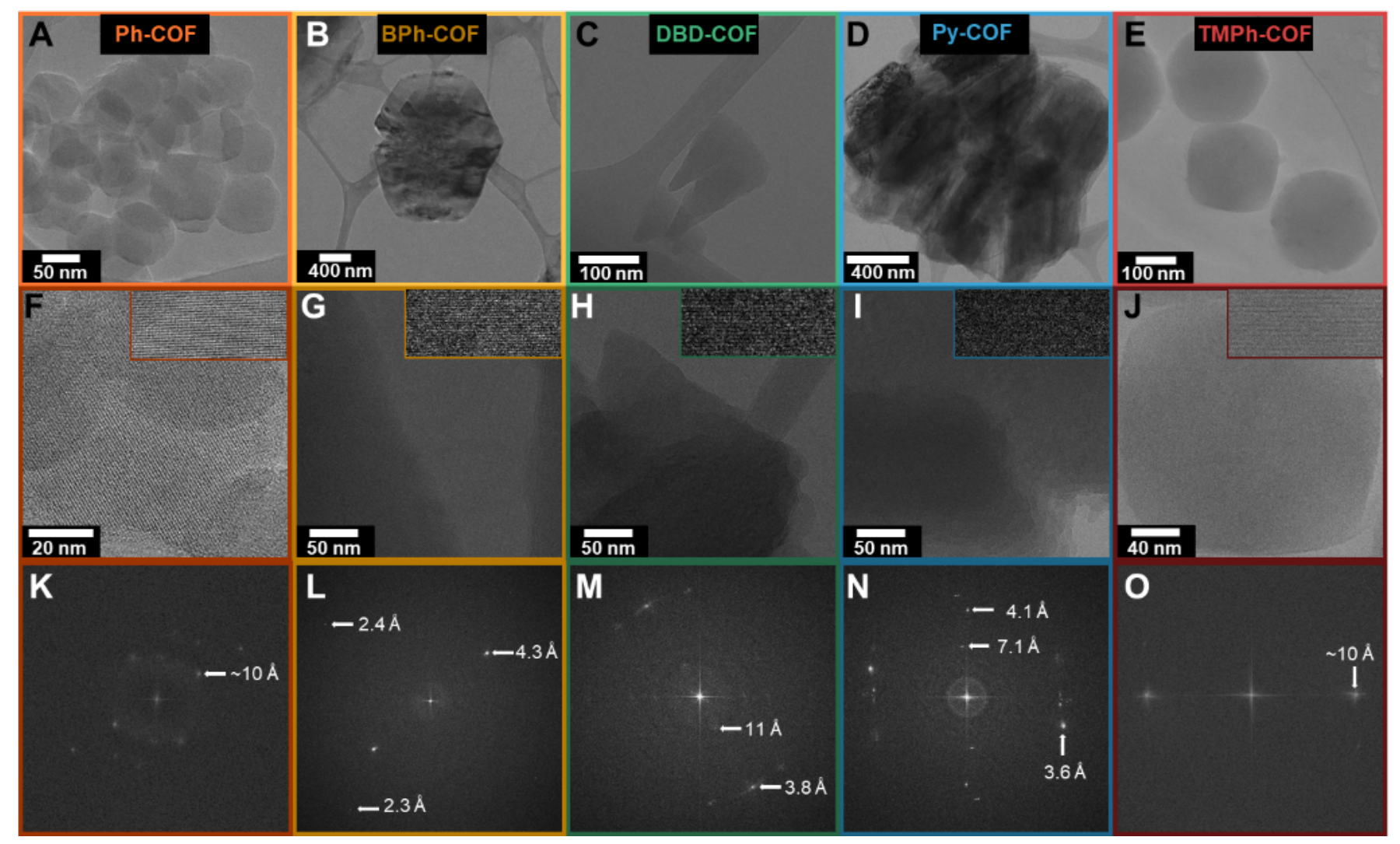

Figure 4. Orange: Ph-COF; Yellow: BPh-COF; Green: DBD-COF; Blue: Py-COF; Red: TMPh-COF. A-E) Low-resolution TEM images of COF particles. F-J) High-resolution TEM images of COF particles. Inset; lattice fringes of COF particles. K-O) FFTs of COF particles.

By TEM, 2D COFs are visible in micrographs as layered single-crystallites with sizes in agreement with DLS measurements (Figures 4A-E and S17-S21). When the images of COF crystallites (Figure 4F-J) were subjected to a fast Fourier transform (FFT) (Figure 4K-4O), we found that all COFs produced a set of symmetric point features rather than ring patterns. This supports the existence of COF crystallites as single-crystalline particles when suspended, as was observed previously for colloidal boronate ester-linked 2D COFs. ${ }^{19}$ For Ph-COF, discrete particles agglomerated upon drop-casting, but they maintained their hexagonal morphology with clearly visible lattice fringes extending from one end of the particles to the other, confirming the single-crystalline nature of the Ph-COF dispersed crystallites (Figure 4A). For Ph-COF, FFT images exhibited a preeminent lattice spacing of $10 \AA\left(d_{110}\right)$. This spacing is consistent with structural models of Ph-COF sheets stacked as alternating offset layers. ${ }^{23} \mathbf{P y}-\mathbf{C O F}$ appeared as agglomerated hexagonally faceted particles with lattice fringes observed at $7.1 \AA\left(d_{200}\right)$, consistent with the in solvo XRD patterns of these materials. In addition to the $d_{200}$ feature, the FFT pattern of Py-COF also exhibited $d_{310}$ features at $4.1 \AA$. Similarly, the well-dispersed hexagonal BPh-COF particles have visible higher-order lattice fringes $\left(d_{400}\right)$, consistent with their XRD pattern. For these three materials, a set of spots at $90^{\circ}$ to the in-plane diffraction features is observed which is in accordance with the expected interlayer distance of 3-4 $\AA$ for van der Waals heterostructures. These $d_{001}$ spacings suggests 
the interlayer distance associated with BPh-COF is smaller than that associated with Py-COF. We attribute this difference to a synchronized offset stacking of alternating phenyl rings that has been reported in other COF systems. ${ }^{37}$ In contrast, such stacking is not possible in Py-COF because of its forced planarity. DBD-COF particles were present as predominantly trigonal particles. Furthermore, the observed preeminent lattice spacing of $11 \AA$ is consistent with the model of these crystallites $\left(d_{110}\right)$ generated through Pawley refinement of the experimentally collected X-ray diffraction patterns (Figure S29-S32). We expect that the prominence of the $d_{210}$ lattice spacing in these crystallites is related to the offset structure assigned from the in solvo XRD patterns. The interlayer arrangement of 2D COF sheets seen here adds to a growing body of evidence that interlayer arrangements of polymer sheets are governed by an array of intermolecular forces. ${ }^{37-41}$ Finally, the 3D TMPh-COF crystals were observed to be regularly faceted crystals with a preeminent lattice spacing of $10 \AA$. This observation is consistent with their previously reported non-interpenetrated diamondoid structure. ${ }^{35}$ Taken together, TEM imaging demonstrates that a second class of 2D COFs and 3D COFs can be synthesized as single-crystalline particles using a colloidal stabilization approach. Furthermore, these results indicate that emerging TEM technologies, such as direct electron detectors facilitating low-dose imaging, are promising tools in the exploration of interlayer arrangements of 2D polymer sheets and electron beam sensitive materials more generally. This interlayer arrangement remains challenging to observe using other characterization methods but is vastly important for a suite of emergent material properties.

Another limitation of precipitated polycrystalline 2D COF powders is the difficulty associated with characterizing their intrinsic optical or electronic properties because of their propensity to scatter light. ${ }^{42}$ However, because 2D materials are of interest for many electronic applications, it is of paramount importance to explore the optoelectronic nature of these materials. During the course of our investigation, we observed that all of the colloidal 2D COF suspensions were noticeably more fluorescent than their boronic acid monomers at the same fluorophore concentrations (Figure 5). Motivated by the results of our previous investigations into enhanced optical characterization of 2D COF colloids, ${ }^{19,}{ }^{43}$ we set out to explore the origin of enhanced nanocrystal fluorescence using EEMS. While EEMS showed that all 2D COF colloids were more emissive than their respective monomers, we chose to focus our investigation on the highly fluorescent Py-COF and DBD-COF materials. 


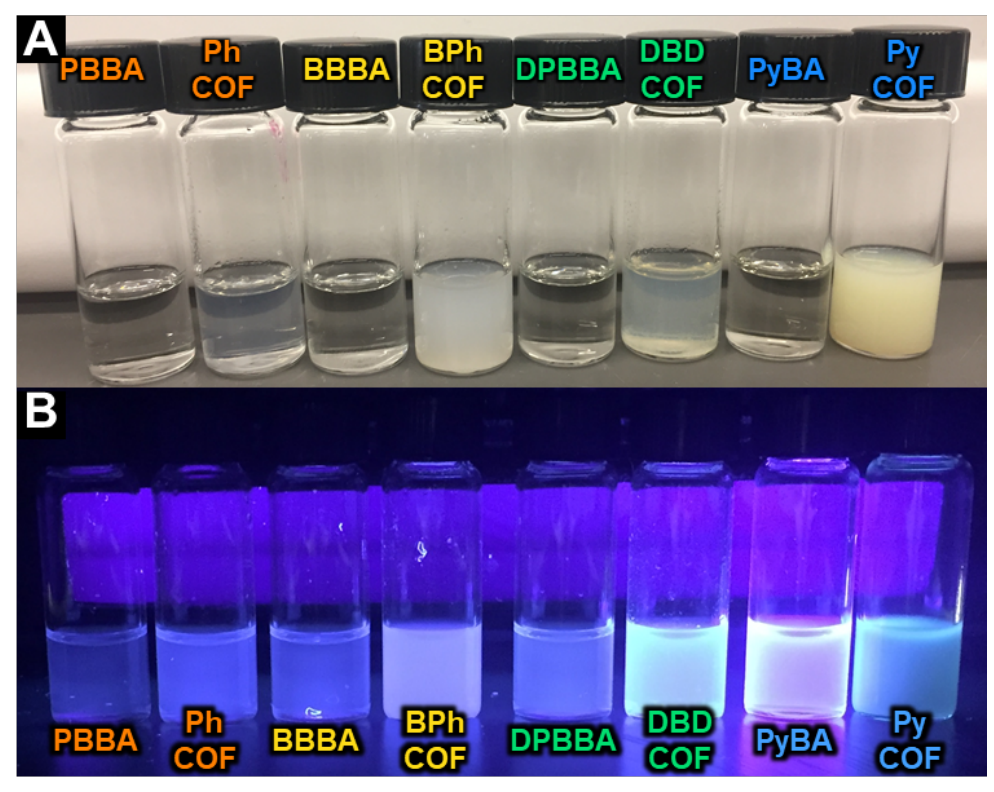

Figure 5. Orange: Ph-COF; Yellow: BPh-COF; Green: DBD-COF; Blue: Py-COF. Photographs of COF monomer solutions and their resultant COF nanocrystals A) under natural light and B) under UV irradiation.

EEMS shows that both DBD-COF (Figure 6A) and its respective DPBBA monomer have absorbance maxima at $365 \mathrm{~nm}$ (Figure 6C). However, the emission of DBD-COF at this wavelength is an order of magnitude more fluorescent than the DPBBA monomer (Figure 6C). This electronic communication was surprising because boroxines are not conjugated and therefore not thought to allow electronic coupling. ${ }^{44}$ However, the difference in monomer and COF fluorescence seen here indicates there is some electronic coupling between chromophores in the COF structure. This behavior is consistent with the understanding that intersheet diphenylbutadiyne chromophores undergo aggregation-induced fluorescence. ${ }^{45-46}$ During the polymerization of DBD-COF, molecular chromophores are brought into close proximity, similar to their aggregation-induced assembly in previous reports, which restricts their molecular motion and enhances their fluorescence quantum yield.

Py-COF was also noteworthy in our initial observations because its fluorescence under UV irradiation changed color significantly when compared to its monomer, PyBA (Figure 5B). Similar to the DBD-COF, the local excited-state emission of Py-COF broadened (Figure 6D), which we attribute to perturbed electronic states of the pyrene chromophores. EEMS of Py-COF shows that the emission blue-shifts $50 \mathrm{~nm}$ relative to the monomer at an excitation of $325 \mathrm{~nm}$ (Figure 6D). This behavior is indicative of plane-plane stacking, so Py-COF can be assigned as an H-aggregated structure. ${ }^{47-48}$ Furthermore, Py-COF has an additional emission feature at $435 \mathrm{~nm}$, which is consistent with the formation of an exciplex structure and has been observed in previous reports of molecular pyrenes (Figure 6E). ${ }^{49}$ This observation is consistent with the assignment of Py-COF as an eclipsed structure having inter-sheet pyrenes in physical contact. Taken together, these results show how electronic communication in framework materials can occur in stacked systems even when not conjugated through 
covalent bonds. We expect that using nonconjugated COFs to exploit through-space electronic communication will feature prominently in future COF systems.
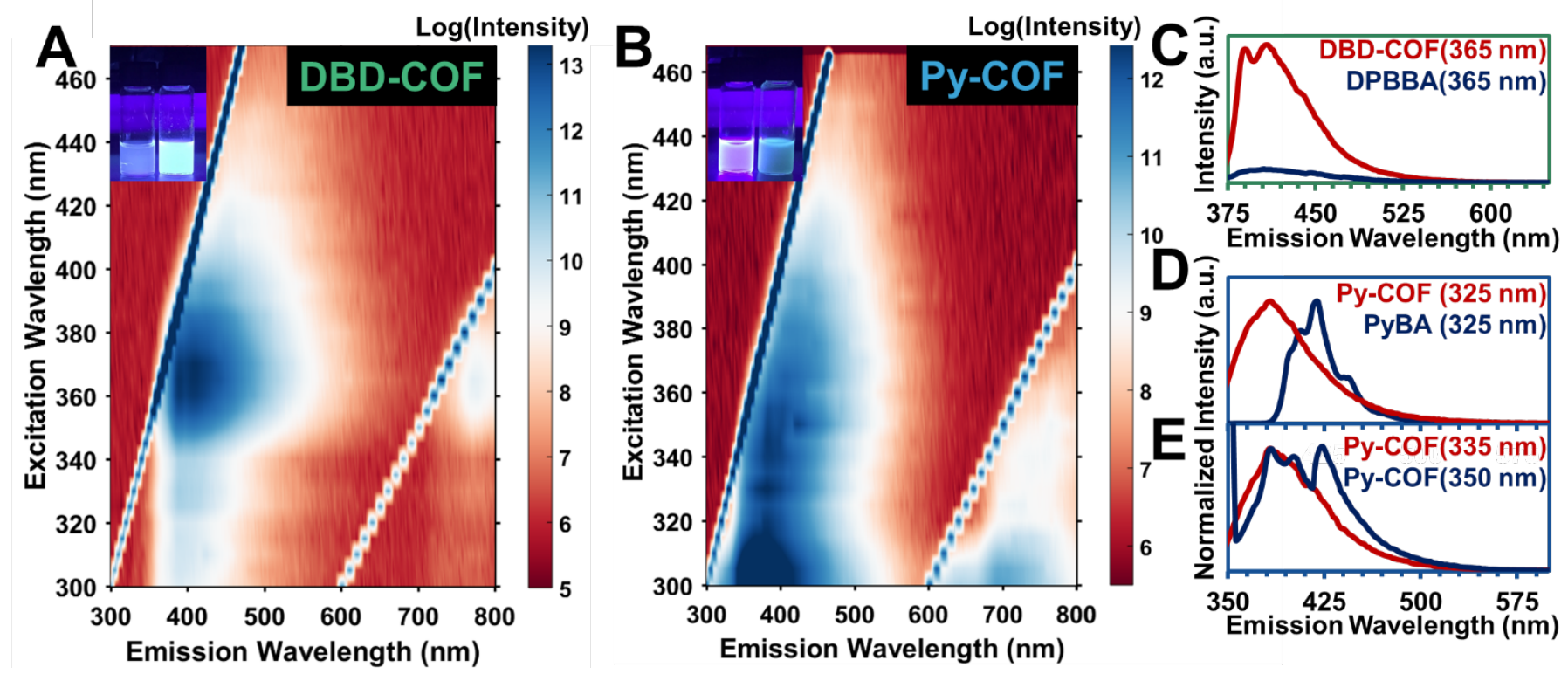

Figure 6. A) EEMS of DBD-COF. Inset; optical image of DPBBA monomer (left) and DBD-COF (Right) under UV irradiation B) EEMS of Py-COF. Inset; optical image of PyBA monomer (left) and Py-COF (Right) under UV irradiation. C) Emission of DBD-COF compared to the DPBBA monomer at the same concentration. D) Normalized emission intensity of Py-COF compared to the PyBA monomer showing a blue shift and broadening of the emission spectra. E) Normalized emission of Py-COF under two excitation wavelengths showing the emergence of an excimer emission $(430 \mathrm{~nm})$.

\section{Conclusion}

2D COFs are promising candidates for many applications, but their investigation and utility has been greatly hampered by the isolation of these materials as weakly polycrystalline aggregates. In the interest of adding 2D COFs to the ever-expanding suite of functional 2D materials, it is imperative to improve their quality and level of characterization, including exploration of their optical properties. The above results demonstrate that colloidal stabilization of boroxine-linked frameworks occurs in the presence of electron-donating solvents. This strategy is amenable to the synthesis of four single-crystalline 2D COF materials, including two newly synthesized systems. Further, we show how this strategy can be topologically expanded to include a 3D COF. Synchrotron XRD and HR-TEM provided insight into the interlayer arrangement of these materials and confirmed their single-crystalline nature. Furthermore, stabilizing COFs as colloidal suspensions allowed us to probe the intrinsic optical properties of these materials with EEMS. The high-quality of the spectroscopy of these materials allowed us to assign the origin of the optical features to the formation of $\pi$-exciplexes, which is indicative of through-space electronic communication of chromophores brought into close proximity in the COF structure. We expect that future colloidal COFs will prove to be a versatile platform to investigate emergent optoelectronic 
phenomena in 2D polymer systems, such as triplet up-conversion, singlet-singlet annihilation, or photoredox processes. ${ }^{50-52}$ Understanding these processes, coupled with the high-quality materials this strategy enables, will ultimately facilitate the incorporation of $2 \mathrm{D}$ polymers into relevant optoelectronic devices.

\section{Acknowledgments}

We thank the Army Research Office for a Multidisciplinary University Research Initiatives (MURI) award under grant W911NF-15-1-0447. A.M.E. is supported by the National Science Foundation Graduate Research Fellowship under grant DGE-1324585. This material is based upon work supported by the National Science Foundation under Grant No. DMREF-1629383. Use of the Center for Nanoscale Materials Argonne National Laboratory, an Office of Science user facility, was supported by the U.S. Department of Energy, Office of Science, Office of Basic Energy Sciences, under Contract No. DE-AC02-06CH11357. L.R.P. was supported by the National Institute of Biomedical Imaging and Bioengineering of the National Institutes of Health under award number F32EB021859. We thank the cryo-electron microscopy facility supported by Prof. Timothy Baker (U.C. San Diego) funded by the National Institutes of Health. I. C. is supported by the NSF Graduate Research Fellowship under Grant No. (DGE-1842165). This study made use of the EPIC facility of NUANCE Center at Northwestern University, which has received support from the Soft and Hybrid Nanotechnology Experimental (SHyNE) Resource (NSF ECCS-1542205), the MRSEC program (NSF DMR-1720139) at the Materials Research Center, the Keck Foundation, the State of Illinois, and International Institute for Nanotechnology (IIN). This work also used resources of the Northwestern University Structural Biology Facility, which is generously supported by NCI CCSG P30 CA060553 awarded to the Robert H. Lurie Comprehensive Cancer Center. A.B. acknowledges support from a 3M graduate research fellowship.

\section{References}

1. Fiori, G.; Bonaccorso, F.; Iannaccone, G.; Palacios, T.; Neumaier, D.; Seabaugh, A.; Banerjee, S. K.; Colombo, L., Electronics based on two-dimensional materials. Nat. Nanotechnol. 2014, 9 (10), 768.

2. Xu, M.; Liang, T.; Shi, M.; Chen, H., Graphene-like two-dimensional materials. Chem. Rev. 2013, $113(5), 3766-3798$.

3. Colson, J. W.; Dichtel, W. R., Rationally synthesized two-dimensional polymers. Nat. Chem. 2013, $5(6), 453$.

4. $\quad$ Sakamoto, J.; van Heijst, J.; Lukin, O.; Schlüter, A. D., Two-dimensional polymers: just a dream of synthetic chemists? Angew. Chem. Int. Ed. 2009, 48 (6), 1030-1069.

5. Kissel, P.; Murray, D. J.; Wulftange, W. J.; Catalano, V. J.; King, B. T., A nanoporous twodimensional polymer by single-crystal-to-single-crystal photopolymerization. Nat. Chem. 2014, 6 (9), 774.

6. $\quad$ Evans, A.; Ryder, M. R.; Flanders, N. C.; Vitaku, E.; Chen, L.; Dichtel, W., Buckling of TwoDimensional Covalent Organic Frameworks Under Thermal Stress. Ind. Eng. Chem. Res. 2019, 58 (23), 9883-9887.

7. Feng, X.; Ding, X.; Jiang, D., Covalent organic frameworks. Chem. Soc. Rev. 2012, 41 (18), 60106022 .

8. Kandambeth, S.; Dey, K.; Banerjee, R., Covalent organic frameworks: chemistry beyond the structure. J. Am. Chem. Soc. 2018, 141 (5), 1807-1822.

9. Lyle, S. J.; Waller, P. J.; Yaghi, O. M., Covalent Organic Frameworks: Organic Chemistry Extended into Two and Three Dimensions. Trends Chem. 2019.

10. Jin, Y.; Hu, Y.; Zhang, W., Tessellated multiporous two-dimensional covalent organic frameworks. Nat. Rev. Chem. 2017, 1 (7), 0056. 
11. Wang, H.; Zeng, Z.; Xu, P.; Li, L.; Zeng, G.; Xiao, R.; Tang, Z.; Huang, D.; Tang, L.; Lai, C., Recent progress in covalent organic framework thin films: fabrications, applications and perspectives. Chem. Soc. Rev. 2019, 48 (2), 488-516.

12. Sick, T.; Hufnagel, A. G.; Kampmann, J.; Kondofersky, I.; Calik, M.; Rotter, J. M.; Evans, A.; Döblinger, M.; Herbert, S.; Peters, K., Oriented films of conjugated 2D covalent organic frameworks as photocathodes for water splitting. J. Am. Chem. Soc. 2018, 140 (6), 2085-2092.

13. Thomas, S.; Li, H.; Dasari, R.; Evans, A. M.; Castano, I.; Allen, T.; Reid, O.; Rumbles, G.; Dichtel, W.; Gianneschi, N. C.; Marder, S., Design and synthesis of two-dimensional covalent organic frameworks with four-arm cores: prediction of remarkable ambipolar charge-transport properties. Mater. Horizons 2019.

14. Corcos, A.; Levato, G.; Jiang, Z.; Evans, A.; Livingston, A.; Mariñas, B.; Dichtel, W., Reducing the Pore Size of Covalent Organic Frameworks in Thin-Film Composite Membranes Enhances Solute Rejection. ChemRxiv 2019.

15. Ma, T.; Kapustin, E. A.; Yin, S. X.; Liang, L.; Zhou, Z.; Niu, J.; Li, L.-H.; Wang, Y.; Su, J.; Li, J., Single-crystal x-ray diffraction structures of covalent organic frameworks. Science 2018, 361 (6397), 48-52.

16. Nguyen, V.; Grünwald, M., Microscopic origins of poor crystallinity in the synthesis of covalent organic framework COF-5. J. Am. Chem. Soc. 2018, 140 (9), 3306-3311.

17. Smith, B. J.; Hwang, N.; Chavez, A. D.; Novotney, J. L.; Dichtel, W. R., Growth rates and water stability of 2D boronate ester covalent organic frameworks. Chem. Comm. 2015, 51 (35), 7532-7535.

18. Li, R. L.; Flanders, N. C.; Evans, A. M.; Ji, W.; Castano, I.; Chen, L. X.; Gianneschi, N. C.; Dichtel, W. R., Controlled growth of imine-linked two-dimensional covalent organic framework nanoparticles. Chem. Sci. 2019, 10 (13), 3796-3801.

19. Evans, A. M.; Parent, L. R.; Flanders, N. C.; Bisbey, R. P.; Vitaku, E.; Kirschner, M. S.; Schaller, R. D.; Chen, L. X.; Gianneschi, N. C.; Dichtel, W. R., Seeded growth of single-crystal two-dimensional covalent organic frameworks. Science 2018, 361 (6397), 52-57.

20. Smith, B. J.; Parent, L. R.; Overholts, A. C.; Beaucage, P. A.; Bisbey, R. P.; Chavez, A. D.; Hwang, N.; Park, C.; Evans, A. M.; Gianneschi, N. C., Colloidal covalent organic frameworks. ACS Cent. Sci. 2017, 3 (1), 58-65.

21. Körzdörfer, T.; Bredas, J.-L., Organic electronic materials: recent advances in the DFT description of the ground and excited states using tuned range-separated hybrid functionals. Acc. Chem. Res. 2014, 47 (11), 3284-3291.

22. Cornil, J.; Lemaur, V.; Calbert, J. P.; Brédas, J. L., Charge transport in discotic liquid crystals: a molecular scale description. Adv. Mater. 2002, 14 (10), 726-729.

23. Cote, A. P.; Benin, A. I.; Ockwig, N. W.; O'keeffe, M.; Matzger, A. J.; Yaghi, O. M., Porous, crystalline, covalent organic frameworks. Science 2005, 310 (5751), 1166-1170.

24. Fischer, A.; Galloway, W.; Vaughan, J., 695. Structure and reactivity in the pyridine series. Part I. Acid dissociation constants of pyridinium ions. J. Am. Chem. Soc. 1964, 3591-3596.

25. Du, Y.; Mao, K.; Kamakoti, P.; Wooler, B.; Cundy, S.; Li, Q.; Ravikovitch, P.; Calabro, D., The effects of pyridine on the structure of B-COFs and the underlying mechanism. J. Mater. Chem. A 2013, 1 (42), 13171-13178.

26. Du, Y.; Mao, K.; Kamakoti, P.; Ravikovitch, P.; Paur, C.; Cundy, S.; Li, Q.; Calabro, D., Experimental and computational studies of pyridine-assisted post-synthesis modified air stable covalentorganic frameworks. Chem. Comm. 2012, 48 (38), 4606-4608.

27. Du, Y.; Calabro, D.; Wooler, B.; Kortunov, P.; Li, Q.; Cundy, S.; Mao, K., One step facile synthesis of amine-functionalized COF-1 with enhanced hydrostability. Chem. Mater. 2015, 27 (5), $1445-$ 1447.

28. Mayoral, A.; Barron, H.; Estrada-Salas, R.; Vazquez-Duran, A.; José-Yacamán, M., Nanoparticle stability from the nano to the meso interval. Nanoscale 2010, 2 (3), 335-342.

29. Li, H.; Evans, A.; Castano, I.; Strauss, M.; Dichtel, W.; Bredas, J.-L., Nucleation-Elongation Dynamics of Two-Dimensional Covalent Organic Frameworks. ChemRxiv 2019. 
30. Berne, B. J.; Pecora, R., Dynamic light scattering: with applications to chemistry, biology, and physics. Courier Corporation: 2000.

31. Flory, P. J., Fundamental principles of condensation polymerization. Chem. Rev. 1946, 39 (1), 137-197.

32. Heller, W.; Klevens, H.; Oppenheimer, H., The determination of particle sizes from Tyndall spectra. J. Chem. Phys. 1946, 14 (9), 566-567.

33. Daugherty, M. C.; Vitaku, E.; Li, R. L.; Evans, A. M.; Chavez, A. D.; Dichtel, W. R., Improved synthesis of $\beta$-ketoenamine-linked covalent organic frameworks via monomer exchange reactions. Chem. Comm. 2019, 55 (18), 2680-2683.

34. Spitler, E. L.; Koo, B. T.; Novotney, J. L.; Colson, J. W.; Uribe-Romo, F. J.; Gutierrez, G. D.; Clancy, P.; Dichtel, W. R., A 2D covalent organic framework with 4.7-nm pores and insight into its interlayer stacking. J. Am. Chem. Soc. 2011, 133 (48), 19416-19421.

35. El-Kaderi, H. M.; Hunt, J. R.; Mendoza-Cortés, J. L.; Côté, A. P.; Taylor, R. E.; O'keeffe, M.; Yaghi, O. M., Designed synthesis of 3D covalent organic frameworks. Science 2007, 316 (5822), 268272.

36. Haase, F.; Troschke, E.; Savasci, G.; Banerjee, T.; Duppel, V.; Dörfler, S.; Grundei, M. M.; Burow, A. M.; Ochsenfeld, C.; Kaskel, S., Topochemical conversion of an imine-into a thiazole-linked covalent organic framework enabling real structure analysis. Nat. Comm. 2018, 9.

37. Auras, F.; Ascherl, L.; Hakimioun, A. H.; Margraf, J. T.; Hanusch, F. C.; Reuter, S.; Bessinger, D.; Döblinger, M.; Hettstedt, C.; Karaghiosoff, K., Synchronized offset stacking: A concept for growing large-domain and highly crystalline $2 \mathrm{~d}$ covalent organic frameworks. J. Am. Chem. Soc. 2016, 138 (51), 16703-16710.

38. Chavez, A. D.; Evans, A. M.; Flanders, N. C.; Bisbey, R. P.; Vitaku, E.; Chen, L. X.; Dichtel, W. R., Equilibration of Imine-Linked Polymers to Hexagonal Macrocycles Driven by Self-Assembly. Chem.: Eur. J. 2018, 24 (16), 3989-3993.

39. Sun, C.; Shen, M.; Chavez, A. D.; Evans, A. M.; Liu, X.; Harutyunyan, B.; Flanders, N. C.; Hersam, M. C.; Bedzyk, M. J.; de la Cruz, M. O.; Dichtel, W., High aspect ratio nanotubes assembled from macrocyclic iminium salts. Proc. Natl. Acad. Sci. U.S.A 2018, 115 (36), 8883-8888.

40. Braunecker, W. A.; Hurst, K. E.; Ray, K. G.; Owczarczyk, Z. R.; Martinez, M. B.; Leick, N.; Keuhlen, A.; Sellinger, A.; Johnson, J. C., Phenyl/perfluorophenyl stacking interactions enhance structural order in two-dimensional covalent organic frameworks. Cryst. Growth Des. 2018, 18 (7), 41604166.

41. Wu, X.; Han, X.; Liu, Y.; Liu, Y.; Cui, Y., Control interlayer stacking and chemical stability of two-dimensional covalent organic frameworks via steric tuning. J. Am. Chem. Soc. 2018, 140 (47), 1612416133.

42. Novoselov, K.; Mishchenko, A.; Carvalho, A.; Neto, A. C., 2D materials and van der Waals heterostructures. Science 2016, 353 (6298), aac9439.

43. Kim, T. W.; Jun, S.; Ha, Y.; Yadav, R. K.; Kumar, A.; Yoo, C.-Y.; Oh, I.; Lim, H.-K.; Shin, J. W.; Ryoo, R., Ultrafast charge transfer coupled with lattice phonons in two-dimensional covalent organic frameworks. Nat. Comm. 2019, 10 (1), 1873.

44. Korich, A. L.; Iovine, P. M., Boroxine chemistry and applications: A perspective. Dalton Trans. 2010, 39 (6), 1423-1431.

45. Pati, A. K.; Jana, R.; Gharpure, S. J.; Mishra, A. K., Photophysics of Diphenylbutadiynes in Water, Acetonitrile-Water, and Acetonitrile Solvent Systems: Application to Single Component White Light Emission. J. Phys. Chem. A 2016, 120 (29), 5826-5837.

46. Pati, A. K.; Gharpure, S. J.; Mishra, A. K., Contrasting Solid-state fluorescence of diynes with small and large aryl substituents: crystal packing dependence and stimuli-responsive fluorescence switching. J. Phys. Chem. A 2015, 119 (42), 10481-10493.

47. Kasha, M.; Rawls, H. R.; El-Bayoumi, M. A., The exciton model in molecular spectroscopy. Pure Appl. Chem. 1965, 11 (3-4), 371-392. 
48. Spano, F. C.; Silva, C., H-and J-aggregate behavior in polymeric semiconductors. Annu. Rev. Phys. Chem. 2014, 65, 477-500.

49. Gai, L.; Chen, H.; Zou, B.; Lu, H.; Lai, G.; Li, Z.; Shen, Z., Ratiometric fluorescence chemodosimeters for fluoride anion based on pyrene excimer/monomer transformation. Chem. Comm. 2012, 48 (87), 10721-10723.

50. Monahan, N.; Zhu, X.-Y., Charge transfer-mediated singlet fission. Annu. Rev. Phys. Chem. 2015, 66, 601-618.

51. Goldschmidt, J. C.; Fischer, S., Upconversion for photovoltaics-a review of materials, devices and concepts for performance enhancement. Adv. Opt. Mater. 2015, 3 (4), 510-535.

52. Liu, X.; Yan, C.-H.; Capobianco, J. A., Photon upconversion nanomaterials. Chem. Soc. Rev. 2015, 44 (6), 1299-1301. 


\title{
Supporting Information
}

\section{Emissive Single-Crystalline Boroxine-Linked Colloidal Covalent Organic Frameworks}

\author{
Austin M. Evans, ${ }^{1}$ Ioannina Castano, ${ }^{1}$ Alexandra Brumberg, ${ }^{1}$ Lucas R. Parent, ${ }^{1,2,3}$ Amanda R. Corcos, ${ }^{1+}$ \\ Rebecca L. Li, ${ }^{1}$ Nathan C. Flanders, ${ }^{1}$ David J. Gosztola,${ }^{7}$ Nathan C. Gianneschi, ${ }^{1-6}$ Richard D. Schaller, ${ }^{1,7}$ \\ William R. Dichtel ${ }^{1,4 *}$ \\ ${ }^{1}$ Department of Chemistry, Northwestern University, Evanston, IL 60208, USA. \\ ${ }^{2}$ Department of Materials Science and Engineering, Northwestern University, Evanston, IL 60208, USA. \\ ${ }^{3}$ Department of Biomedical Engineering, Northwestern University, Evanston, IL 60208, USA. \\ ${ }^{4}$ International Institute for Nanotechnology, Northwestern University, Evanston, IL 60208, USA \\ ${ }^{5}$ Simpson Querrey Institute, Northwestern University, Evanston, IL 60208, USA \\ ${ }^{6}$ Chemistry of Life Processes Institute, Northwestern University, Evanston, IL 60208, USA \\ ${ }^{7}$ Center for Nanoscale Materials, Argonne National Laboratory, Argonne, IL 60439, USA. \\ *Current Address: AAAS Science \& Technology Policy Fellow at U.S. Department of State, Washington, D.C. \\ *Email: wdichtel@,northwestern.edu
}

\section{Table of Contents}

\begin{tabular}{|c|c|c|}
\hline Section & Title and Contents & Pages \\
\hline $\mathbf{I}$ & Materials and Methods & S3 - S5 \\
\hline \multirow[t]{3}{*}{ II } & Synthetic Procedures for Covalent Organic Framework Monomers & $\mathrm{S} 6-\mathrm{S} 8$ \\
\hline & Scheme S1. Synthesis of 2,7-Pyrenebisboronic acid & \\
\hline & $\begin{array}{l}\text { Scheme S2. Synthesis of Synthesis of 4,4'-Diphenylbutadiyne boronic acid } \\
\text { Scheme S3. Synthesis of Synthesis of Tetra(4-dihydroxyborylphenyl)methane }\end{array}$ & \\
\hline \multirow[t]{5}{*}{ III } & $\begin{array}{c}\text { Nuclear Magnetic Resonance of Covalent Organic Framework Monomers } \\
\text { Figure S1. }{ }^{1} \mathrm{H} \text { NMR of 2,7-Pyrenebisboronic acid DMSO-d } \mathrm{d}_{6}\end{array}$ & S9 - S13 \\
\hline & Figure S2. ${ }^{1} \mathrm{H}$ NMR of 4,4'-Diphenylbutadiyne pinacoleborane in $\mathrm{CDCl}_{3}$ & \\
\hline & Figure S3. ${ }^{1} \mathrm{H}$ NMR of $4,4^{\prime}$-Diphenylbutadiyne boronic acid in DMSO- $\mathrm{d}_{6}$ & \\
\hline & Figure S4. ${ }^{1} \mathrm{H}$ NMR of Tetra(4-bromophenyl)methane in $\mathrm{CDCl}_{3}$ & \\
\hline & Figure S5. ${ }^{1} \mathrm{H}$ NMR of Tetra(4-dihydroxyborylphenyl)methane in DMSO-d 6 & \\
\hline \multirow[t]{6}{*}{ IV } & Covalent Organic Framework Structures & S14 - S16 \\
\hline & Figure S6. Structure of $\mathbf{P h}-\mathrm{COF}$ & \\
\hline & Figure S7. Structure of BPh-COF & \\
\hline & Figure S8. Structure of DBD-COF & \\
\hline & Figure S9. Structure of Py-COF & \\
\hline & Figure S10. Structure of TMPh-COF & \\
\hline \multirow[t]{6}{*}{$\mathbf{V}$} & Synthesis of Covalent Organic Frameworks & S17 - S19 \\
\hline & Scheme S4. Synthesis of Ph-COF & \\
\hline & Scheme S5. Synthesis of BPh-COF & \\
\hline & Scheme S6. Synthesis of DBD-COF & \\
\hline & Scheme S7. Synthesis of Py-COF & \\
\hline & Scheme S8. Synthesis of TMPh-COF & \\
\hline \multirow[t]{3}{*}{ VI } & $\begin{array}{c}\text { Fourier-Transform Infrared Spectroscopy of Covalent Organic Frameworks } \\
\text { Figure 11. FT-IR of Ph-COF Powder }\end{array}$ & $\mathrm{S} 20-\mathrm{S} 25$ \\
\hline & Figure 12. FT-IR of Ph-COF Colloids & \\
\hline & Figure 13. FT-IR of BPh-COF Colloids & \\
\hline
\end{tabular}


Figure 14. FT-IR of DBD-COF Colloids

Figure 15. FT-IR of Py-COF Colloids

Figure 16. FT-IR of TMPh-COF Colloids

VII

Dynamic Light Scattering of Covalent Organic Frameworks

Figure 17. Dynamic light scattering number distribution of $\mathbf{P h}-\mathbf{C O F}$

Figure 18. Dynamic light scattering number distribution of BPh-COF

Figure 19. Dynamic light scattering number distribution of DBD-COF

Figure 20. Dynamic light scattering number distribution of Py-COF

Figure 21. Dynamic light scattering number distribution of TMPh-COF

VIII X-ray Diffraction of Collected COF Powders

Figure 22. X-ray diffraction of wet $\mathbf{P h - C O F}$

IX COF Structural Models

Figure 23. Structure of $\mathbf{P h}-\mathbf{C O F}$

Figure 24. Structure of $\mathrm{BPh}-\mathrm{COF}$

Figure 25. Structure of DBD-COF

Figure 26. Structure of Py-COF

Figure 27. Structure of TMPh-COF

Figure 28. Structure of TMPh-COF - Zoomed in

$\mathbf{X}$

COF Modeled Diffraction Patterns

Figure S29. BPh-COF Diffraction Patterns

Figure S30. DBD-COF Diffraction Patterns

Figure S31. TMPh-COF Diffraction Patterns

Figure S32. Py-COF Diffraction Patterns

XI

Additional COF Fluorescence

Figure S33. Excitation-Emission Matrix Spectroscopy of Ph-COF

Figure S34. Excitation-Emission Matrix Spectroscopy of BPh-COF

XII

Additional Transmission Electron Microscopy Images

Figure S35. Additional TEM images

Figure S36. Additional High Resolution TEM images

XIII

Fluorescence of COF Colloids versus Powders

Figure S37. Fluorescence Spectroscopy of Py-COF

Figure S38. Fluorescence Spectroscopy of DBD-COF
$\mathrm{S} 26-\mathrm{S} 31$

$\mathrm{S} 33-\mathrm{S} 34$

$\mathrm{S} 35-\mathrm{S} 37$

S38

$\mathrm{S} 39-\mathrm{S} 40$

S41 - S42

S43

XIV References 


\section{Materials and Methods}

Materials. All monomers, solvents, and catalysts were either purchased from commercial sources or prepared following literature reported protocols. All materials were used as received without further purification. Anhydrous THF was obtained from a solvent purification system (JC Myer System).

Solution Nuclear Magnetic Resonance. ${ }^{1} \mathrm{H}$ NMR spectra were acquired on a $400 \mathrm{MHz}$ Agilent DD MR-400 system or Bruker Avance III $500 \mathrm{MHz}$ spectrometer and recorded at $25^{\circ} \mathrm{C}$. All chemical shifts were calibrated using residual solvent as internal reference $\left(\mathrm{CDCl}_{3}: 7.26 \mathrm{ppm}\right.$ for ${ }^{1} \mathrm{H}$ NMR. DMSO: 2.5 ppm for ${ }^{1} \mathrm{H}$ NMR).

Dynamic Light Scattering. Dynamic light scattering (DLS) data were collected using a Malvern Zetasizer equipped with a $633 \mathrm{~nm}$ He-Ne $5 \mathrm{~mW}$ laser in a quartz cuvette with a $10 \mathrm{~mm}$ pathlength at room temperature. Data was analyzed using the proprietary Zetasizer software.

Fourier-Transform Infrared Spectroscopy. Infrared spectra were collected on a Nicolet iS10 FT-IR spectrometer equipped with a ZnSe crystal in attenuated total reflection mode. Spectra were background subtracted to remove contribution from atmospheric species. Spectra as shown were not corrected or calibrated to any IR feature.

In solvo X-ray Diffraction. Small- and wide-angle X-ray scattering (SAXS/WAXS) patterns were collected at Argonne National Lab's (ANL) Advanced Photon Source (APS) at both sectors 5-IDD (DND-CAT) and 12-ID-D with a capillary transmission geometry. Experiments conducted at 12-ID-D were collected at a beam energy of $12 \mathrm{keV}$ and experiments conducted at 5-ID-D were collected at a beam energy of $13.3 \mathrm{keV}$. Individual frames were collected on a set of Pilatus detectors, which were then summed and radially integrated to produce a linear XRD pattern using proprietary software available at the APS. Scattering intensity is reported as a function of the modulus of the scattering vector $q$, related to the scattering angle $2 \theta$ by the equation $q=(4 \pi / \lambda) \sin$ $\theta$, where $\lambda$ is the X-ray wavelength. The sample-to-detector distance was adjusted to measure across relevant detection ranges. Capillary experiments were conducted using $2.0 \mathrm{~mm}$ OD borosilicate capillaries with $0.2 \mathrm{~mm}$ wall thicknesses purchased from Hilgenberg $\mathrm{GmbH}$.

Grazing-Incidence X-ray Diffraction. As noted in the text, several reported X-ray scattering patterns were collected by drop casting undiluted samples of COF nanocrystals onto silicon wafers and allowing the solvent to evaporate. GIWAXS measurements were then performed at Advanced Photon Source at Argonne National Laboratory using the 8-ID-E Beamline under vacuum. The crystallites were irradiated until the detector was $80 \%$ of saturated an incidence angle of $0.14^{\circ}$ in vacuum using $10.92 \mathrm{keV}(1.135 \AA$ ) $)$ X-rays. The scattering was recorded on a Pilatus $1 \mathrm{M}$ detector located $228 \mathrm{~mm}$ from the sample. In all cases, significant silicon substrate scatter was observed. The raw images were merged, pixel coordinates were transformed to q-space, line cuts generated using GIXSGUI for Matlab. ${ }^{1}$

COF Modeling and Structural Refinement. Crystal modeling of the COF structures was carried out using the Materials Studio (ver.5.0) suite of programs by Accelrys. ${ }^{2}$ The initial structures were 
constructed piecewise starting with a primitive hexagonal unit cell of a P6 space group. The cell parameter was estimated according to the distance between the center of the vertices for each COF, and c parameter was chosen as $3.35 \AA$, which has been observed for similar materials. ${ }^{3}$ Initially, these structures were estimated to be eclipsed. The structures were optimized using a Geometry Optimization routine including energy minimization with cell parameters optimization, using the parameters from the Universal Force Field. Calculation of the simulated powder diffraction patterns and Pawley refinements were performed in the Materials Studio Reflex Plus Module using a Bragg-Brentano geometry. When the first $\sim 2$ features were seen to align but relative intensities were seen to be inconsistent, we assigned this to a noneclipsed structure. If this was the case, supercells of the crystallites were generated and different stackings $(A B, A B C$, partial $A B$, etc. were attempted). The intensity profile which matched most closely was used to refine. The observed diffraction patterns were subjected to a polynomial background subtraction and then to Pawley refinement wherein peak profile were refined using the Pseudo-Voigt peak shape function and asymmetry was corrected using the Berar-Baldinozzi function. Crystallite size was then estimated by the LeBail method which was Pawley refined to the experimental data.

Transmission Electron Microscopy Sample Preparation for Ph-COF and TMPh-COF. All COF samples ( $\mathrm{Ph}-\mathrm{COF}$ and TMPh-COF) were prepared by drop-casting $\sim 3-4 \mu \mathrm{L}$ (using a micropipette) of the COF sample solution $\left(\mathrm{CH}_{3} \mathrm{CN}\right.$ :1,4-dioxane:mesitylene, 50:40:10 by volume) after synthesis was complete onto lacey-carbon substrate $(\mathrm{Cu}$ mesh) TEM grids (Electron Microscopy Sciences, Hatfield, PA). The droplets of COF sample were allowed to sit on the grids in ambient conditions for $\sim 20-30$ s and were then wicked dry with filter paper. The prepared TEM grids were then stored in a desiccator until they were used for TEM characterization.

Transmission Electron Microscopy for Ph-COF and TMPh-COF. TEM was performed using a FEI (FEI Company, Hillsboro, OR) Polara cryoTEM operating at $200 \mathrm{keV}$ equipped with a Gatan (Roper Technologies, Sarasota, FL) K2 “direct electron" detector (FEG extraction: 4350 V, FEG emission: $108 \mu \mathrm{A}$, spot size 5, $70 \mu \mathrm{m}$ OL aperture, $70 \mu \mathrm{m} \mathrm{CL}$ aperture). The Polara cryoTEM is a cartridge-loaded microscope, where the column is continually maintained at $\mathrm{L}-\mathrm{N}_{2}$ temperature $\left(<-180^{\circ} \mathrm{C}\right)$. The grids that were prepared previously and dried, were loaded into the microscope's cartridge after being submerged in $\mathrm{L}_{-} \mathrm{N}_{2}$, and the cartridge with grids was inserted into the microscope (maintained at $\mathrm{L}-\mathrm{N}_{2}$ temperature). The Polara was aligned for low-dose imaging, measuring the dose rate on the $\mathrm{K} 2$ detector through vacuum (no grid inserted). The dose rate used was $5.12 \mathrm{e}-\AA^{-2} \mathrm{~s}$ for high-magnification (115 kx) images (1.78 $\AA$ pixel size, $3708 \mathrm{x} 3838$ pixels), with an image exposure of time of $5 \mathrm{~s}\left(\sim 25.6 \mathrm{e}-\AA^{-2}\right.$ cumulative dose per image). The dose rate was not directly measured for the low magnification images, but the dose rate was orders magnitude lower for those images than for the high magnification images (1ow magnification images: $1 \mathrm{~s}$ exposure time, $10.1034 \mathrm{~nm}$ pixel size, 1852 x 1918 pixels, binning 2). All image acquisition was performed using SerialEM software which applies auto focusing on adjacent regions of the grid to minimize dose on the sample. ${ }^{4}$

Transmission Electron Microscopy Sample Preparation for BPh-COF, DBD-COF, and PyCOF. All COF samples were prepared by drop-casting $\sim 4 \mu \mathrm{L}$ (using a micropipette) of the COF sample solution $\left(\mathrm{CH}_{3} \mathrm{CN}\right.$ :1,4-dioxane:mesitylene, 50:40:10 by volume) onto lacey-carbon 
substrate (Cu, 400 mesh) TEM grids (Ted Pella, Redding, CA). The droplets of COF sample were allowed to sit on the grids in ambient conditions for $\sim 10 \mathrm{~s}$ and were then wicked dry with filter paper.

Transmission Electron Microscopy for BPh-COF, DBD-COF, and Py-COF. Low magnification TEM was performed using a JEOL (JEOL USA, Inc., Peabody, MA) ARM300 GrandARM TEM operating at $300 \mathrm{keV}$ equipped with a Gatan (Gatan, Inc., Pleasanton, CA) OneView-IS camera (FEG Emission: $15 \mu \mathrm{A}$, spot size 4, $100 \mu \mathrm{m} \mathrm{CL}$ aperture, $40 \mu \mathrm{m}$ objective aperture). All image acquisition was done using the Gatan Microscopy Suite (GMS), Digital Micrograph (Gatan, Inc., Pleasanton, CA).

High resolution TEM was performed using a JEOL (JEOL USA, Inc., Peabody, MA) 3200FS TEM operating at $300 \mathrm{keV}$ equipped with a Gatan (Gatan, Inc., Pleasanton, CA) K2 summit direct electron detector $\left(\sim 5 \mathrm{e}-\AA^{-2} \mathrm{~s}^{-1}\right.$ dose per image) using Leginon where all alignment was done at $4 \mathrm{k}$ or lower. ${ }^{5}$

Fluorescence Spectroscopy Sample Preparation. Monomer solutions were prepared at a concentration of $15 \mathrm{mM}$ in a $4 \mathrm{~mL}$ mixture of $\mathrm{CH}_{3} \mathrm{CN}$ :1,4-dioxane:mesitylene (50:40:10 v/v/v). This monomer solution was then sonicated for five minutes and briefly heated to aid in solvation of the monomers. This $4 \mathrm{~mL}$ sample was split into two fractions of $2 \mathrm{~mL}$ apiece and each sealed. One of these solutions was heated at $70{ }^{\circ} \mathrm{C}$ for 72 hours while the other was left at room temperature. Emission-Excitation Matrix Fluorescence Spectroscopy (EEMS) was performed on all samples.

Emission-Excitation Matrix Spectroscopy. Emission and excitation spectra were recorded on a Horiba Jobin Yvon Fluorolog-3 fluorescence spectrophotometer equipped with a $450 \mathrm{~W}$ Xe lamp, double excitation and double emission monochromators, a digital photon-counting photomultiplier and a secondary InGaAs detector for the NIR range. Correction for variations in lamp intensity over time and wavelength was achieved with a solid-state silicon photodiode as the reference. The spectra were further corrected for variations in photomultiplier response over wavelength and for the path difference between the sample and the reference by multiplication with emission correction curves generated on the instrument. Emission-Excitation Matrix Spectroscopy was conducted on these samples by scanning the emission profile of different excitation wavelengths.

Centrifugation. Centrifugation was conducted using an Eppendorf Centrifuge 5430 V 4.4. Samples were collected in falcon tubes, balanced, and spun for 5 minutes at $7830 \mathrm{rpm}$. This was repeated twice to attempt to remove any small species not firmly collected. 


\section{Synthetic Procedures for Covalent Organic Framework Monomers}

Scheme S1. Synthesis of 2,7-Pyrenebisboronic acid

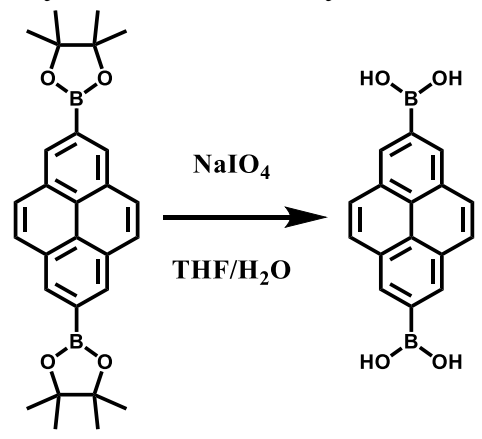

2,7-Pyrenebisboronic acid. This compound was synthesized according to published procedure ${ }^{6}$ but with minor modification. In a $250 \mathrm{~mL}$ round bottom flask, bis(pinacoloto)diboronic ester $(1.00 \mathrm{~g}, 2.20 \mathrm{mmol}$, 1.00 equiv) and sodium periodate $\left(2.84 \mathrm{~g}, 13.4 \mathrm{mmol}, 6.00\right.$ equiv) were dissolved in a THF: $\mathrm{H}_{2} \mathrm{O}$ mixture $(4: 1 \mathrm{v} / \mathrm{v}, 60 \mathrm{~mL})$. This mixture was stirred at room temperature with nitrogen actively bubbling through it for 30 minutes. (NOTE: $\mathrm{HCl}$ was not added to this reaction mixture as was done in previous reports[REF] thereby reducing orange/brown impurities in the final compound.) The reaction vessel was subsequently sealed and allowed to stir for an additional 24 hours. At this point, $60 \mathrm{~mL}$ of $\mathrm{H}_{2} \mathrm{O}$ was added to the reaction mixture, which was then filtered through a Büchener funnel, and the product was washed with an additional $100 \mathrm{~mL}$ of $\mathrm{H}_{2} \mathrm{O}$. This product was then flushed with $100 \mathrm{~mL}$ of diethyl ether. This powder was dried under vacuum for 2 hours to afford a fine white powder $(520 \mathrm{mg}, 82 \%)$. NMR analysis of this product was consistent with a previous report. $^{6}$

Scheme S2. Synthesis of Synthesis of 4,4'-Diphenylbutadiyne boronic acid

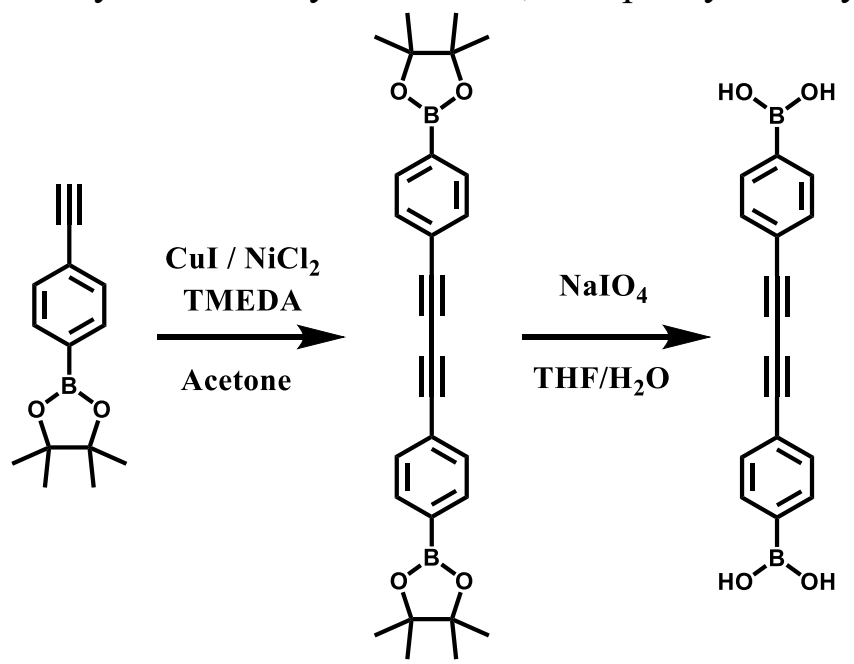

4,4'-Diphenylbutadiyne pinacole borane. The synthesis of this product was adapted from a previous report. $^{7}$ A $100 \mathrm{~mL}$ round bottom flask was charged with $\mathrm{CuI}(0.752 \mathrm{~g}, 3.94 \mathrm{mmol}), \mathrm{NiCl}_{2} \bullet 6 \mathrm{H}_{2} \mathrm{O}(0.936 \mathrm{~g}$, $3.94 \mathrm{mmol})$, and tetramethylethylenediamine (TMEDA) (1.836 g, $15.784 \mathrm{mmol})$. Acetone (50 mL) was added, and as the solids dissolved the mixture became dark green. 4-ethynylbenzeneboronic acid pinacole ester (18 g, $78.8 \mathrm{mmol})$ was added to this mixture, which was then stirred for 16 hours. The solvent was removed by rotary evaporation to yield a green residue that was washed with $\mathrm{H}_{2} \mathrm{O}(300 \mathrm{~mL})$. The resultant solid was isolated, subsequently recrystallized from $\mathrm{CH}_{3} \mathrm{CN}$ as a white solid, collected via filtration, and 
dried under vacuum $(5.25 \mathrm{~g}, 30 \%)$. NMR analysis of this product was consistent with a previous report. ${ }^{7}$

4,4'-Diphenylbutadiyne boronic acid. The synthesis of this compound was adapted from a previous report. ${ }^{8}$ In a $20 \mathrm{~mL}$ scintillation vial, 4,4'-diphenylbutadiyne pinacole borane $(1.00 \mathrm{~g}, 2.20 \mathrm{mmol}, 1.00$ equiv) and sodium periodate $\left(1.0 \mathrm{~g}, 5.00 \mathrm{mmol}, 2.27\right.$ equiv) were dissolved in THF: $\mathrm{H}_{2} \mathrm{O}(4: 1 \mathrm{v} / \mathrm{v}, 12 \mathrm{~mL})$. This mixture was stirred at room temperature with nitrogen actively bubbling through it for 30 minutes, after which $1 \mathrm{M} \mathrm{HCl}(5 \mathrm{~mL})$ was added via syringe. The reaction vessel was subsequently sealed and allowed to stir for an additional 24 hours. At this point, the reaction mixture was poured into $50 \mathrm{~mL}$ of $\mathrm{H}_{2} \mathrm{O}$, filtered through a Büchener funnel, and washed with an additional $100 \mathrm{~mL}$ of $\mathrm{H}_{2} \mathrm{O}$. This product was then flushed with $100 \mathrm{~mL}$ of diethyl ether. This powder was dried under vacuum for 10 minutes to afford a fine white powder (444 mg, 69\%). NMR analysis of this product was consistent with a previous report. ${ }^{7}$

Scheme S3. Synthesis of Synthesis of Tetra(4-dihydroxyborylphenyl)methane

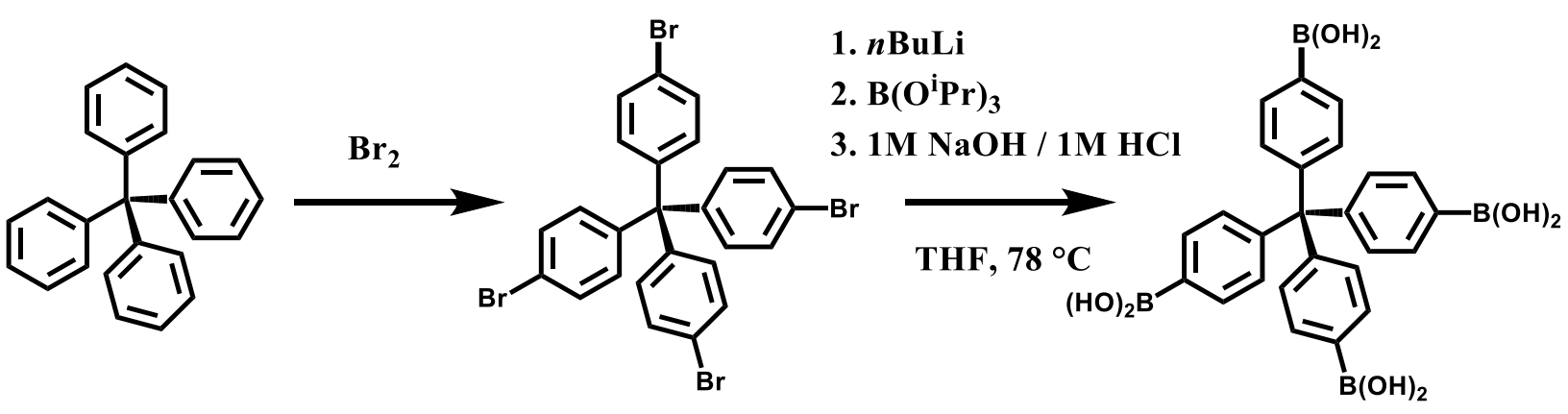

Tetra(4-bromophenyl)methane. The synthesis of this compound was adapted from a previous report. ${ }^{9} \mathrm{~A}$ $100 \mathrm{ml}$ three-necked flask was equipped with a magnetic stir bar and charged with tetraphenylmethane (9 $\mathrm{g}, 25 \mathrm{mmol}$ ). Two of the flask necks were covered with septa and a condenser with a hose at the top was inserted into the third neck, which was attached to a concentrated $\mathrm{Na}_{2} \mathrm{~S}_{2} \mathrm{O}_{3}$ solution. (Note: This was included so as to neutralize any escaped $\mathrm{Br}_{2}$ gas in the following step.) Neat $\mathrm{Br}_{2}(10 \mathrm{~mL}, 0.175 \mathrm{mmol})$ was added slowly via syringe to the stirring mixture through one of the septa, yielding a dark orange slurry that was stirred for an additional 20 minutes. This mixture was poured into cold ethanol $\left(200 \mathrm{~mL},-78^{\circ} \mathrm{C}\right)$. The precipitated solid from this reaction was combined with saturated aqueous $\mathrm{NaHSO}_{3}(80 \mathrm{~mL})$ and filtered and washed with an additional $80 \mathrm{~mL}$ of $\mathrm{NaHSO}_{3}$ two times. At this point, the filtered liquid was transparent. The crude solid was recrystallized from a 1:1 chloroform/ethanol mixture and isolates as a pale yellow solid $(5.85 \mathrm{~g}, 37 \%)$. NMR analysis of this product was consistent with a previous report. ${ }^{9}$

Tetra(4-dihydroxyborylphenyl)methane. The synthesis of this compound was adapted from a previous report. ${ }^{10}$ A solution of tetra(4-bromophenyl)methane $(1.27 \mathrm{~g}, 2.00 \mathrm{mmol})$ in anhydrous THF (125 mL) was stirred at $-78{ }^{\circ} \mathrm{C}$ under a $\mathrm{N}_{2}$ atmosphere. To this, $n$-butyllithium $(6.4 \mathrm{~mL}, 2.5 \mathrm{M} \mathrm{THF}, 16 \mathrm{mmol})$ was added dropwise via cannula through a pre-marked secondary container, after which the solution became cloudy. The resulting mixture continued to stir for 30 minutes at $78{ }^{\circ} \mathrm{C}$, whereupon $\mathrm{B}\left(\mathrm{O}-{ }^{i} \mathrm{Pr}\right)_{3}(5.5 \mathrm{~mL}, 23.8 \mathrm{mmol})$ was added dropwise via cannula through a pre-marked secondary container. The mixture was stirred at -78 ${ }^{\circ} \mathrm{C}$ for an additional 20 minutes and then slowly warmed to room temperature, where it was stirred for an additional 12 hours. After acidification with $1 \mathrm{M} \mathrm{HCl}(25 \mathrm{~mL})$, the mixture was concentrated by partial evaporation of volatiles under reduced pressure, which yielded a white solid. This concentrate was treated 
with $1 \mathrm{M} \mathrm{NaOH}(25 \mathrm{~mL})$ and then filtered. The filtrate was acidified with $1 \mathrm{M} \mathrm{HCl}$ until a white precipitate was observed. The precipitate was collected by filtration, washed with $\mathrm{Et}_{2} \mathrm{O}$, and dried under vacuum to afford tetra(4-dihydroxyborylphenyl)methane as a white solid (0.301 g, 30.4\%). NMR Analysis of this product was consistent with a previous report. ${ }^{10}$ 
III. Nuclear Magnetic Resonance of Covalent Organic Framework Monomers

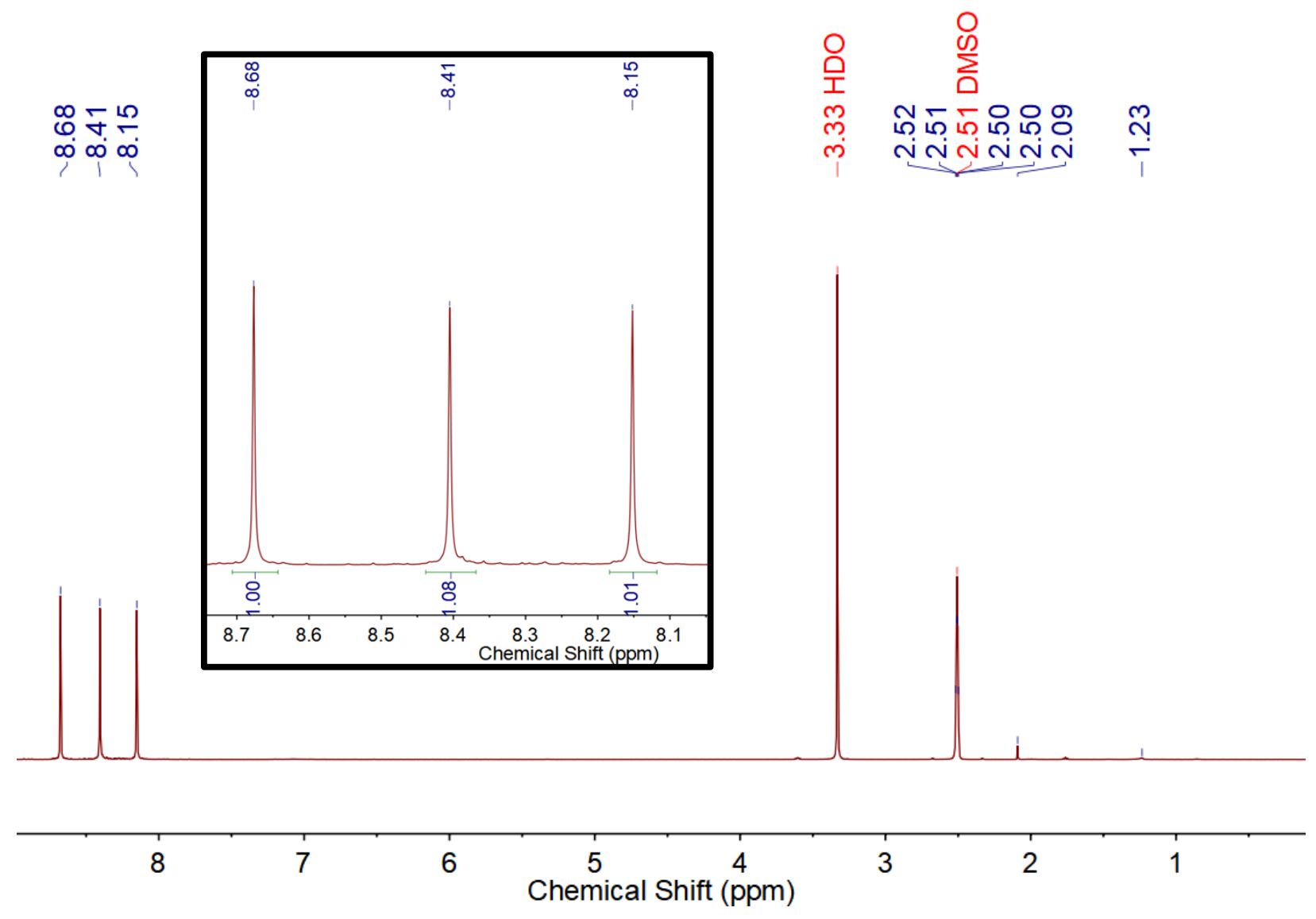

Figure S1. ${ }^{1} \mathrm{H}$ NMR of 2,7-Pyrenebisboronic acid DMSO-d 6 


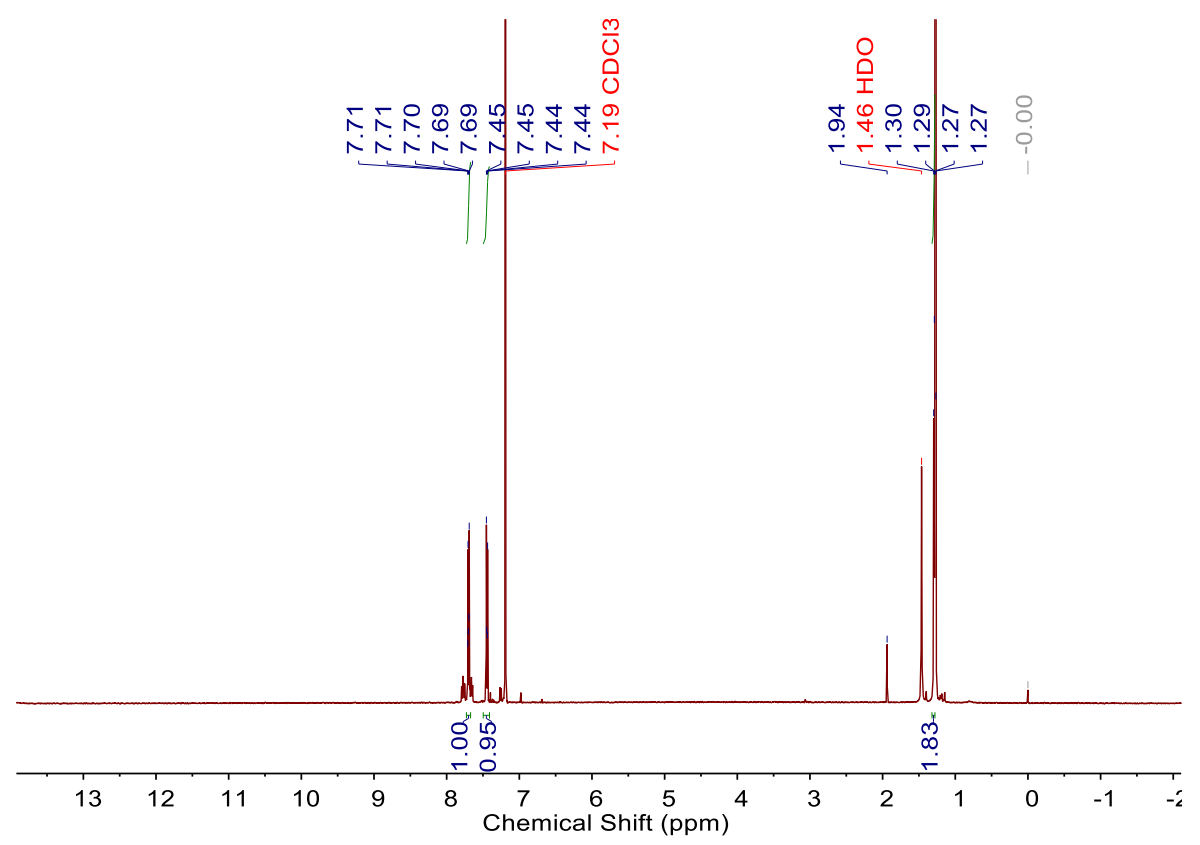

Figure S2. ${ }^{1} \mathrm{H}$ NMR of 4,4'-Diphenylbutadiyne pinacoleborane in $\mathrm{CDCl}_{3}$ 


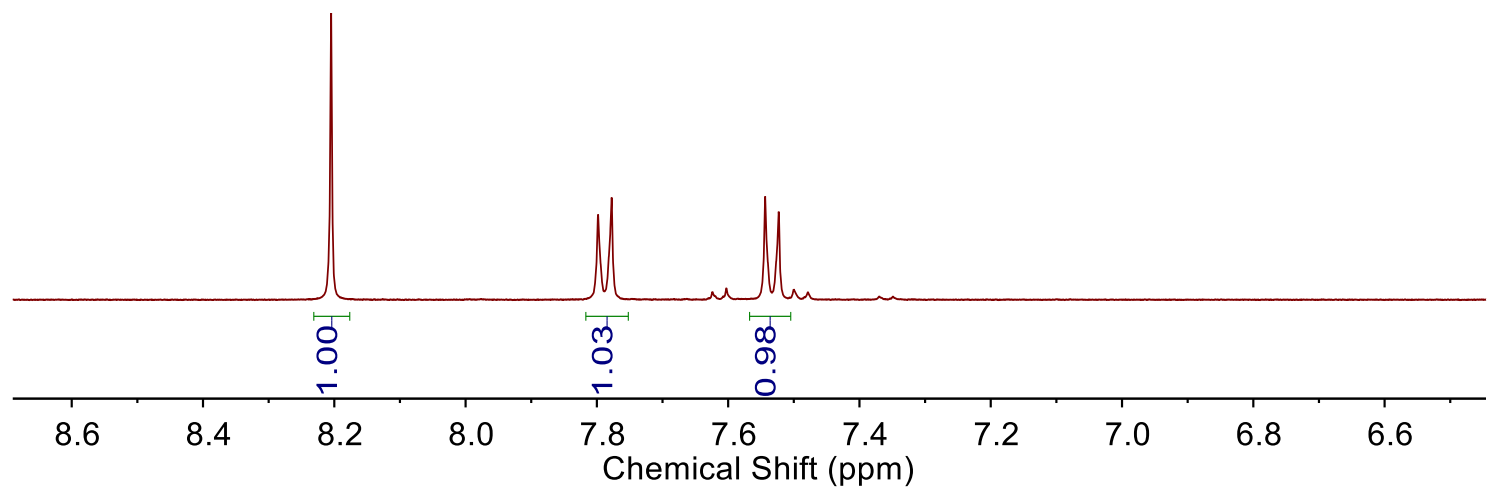

Figure S3. ${ }^{1} \mathrm{H}$ NMR of 4,4'-Diphenylbutadiyne boronic acid in DMSO-d 6 


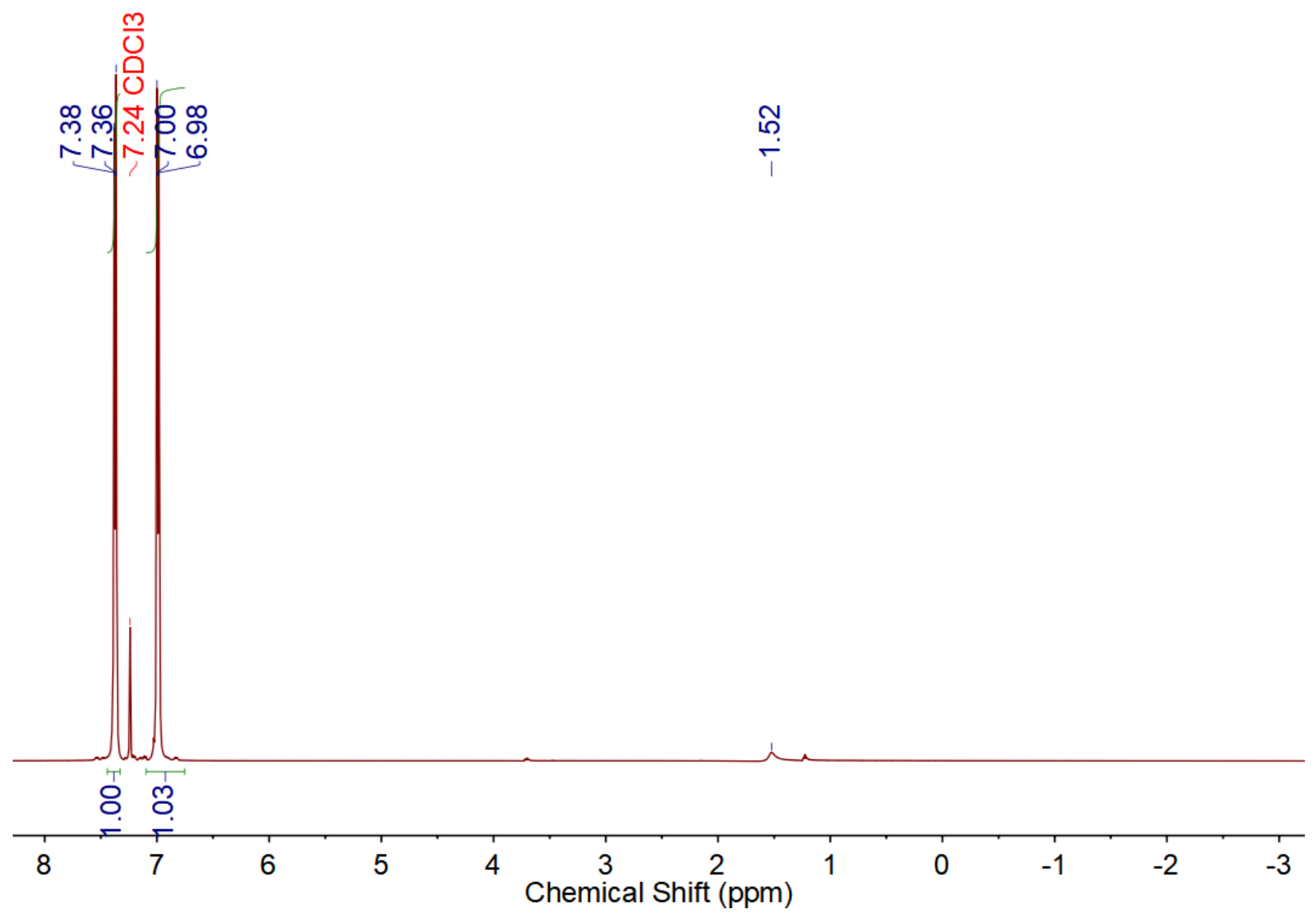

Figure S4. ${ }^{1} \mathrm{H}$ NMR of Tetra(4-bromophenyl)methane in $\mathrm{CDCl}_{3}$ 


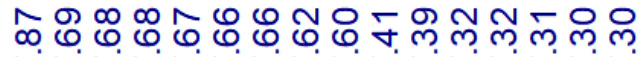

NNNNNNNNNNNN

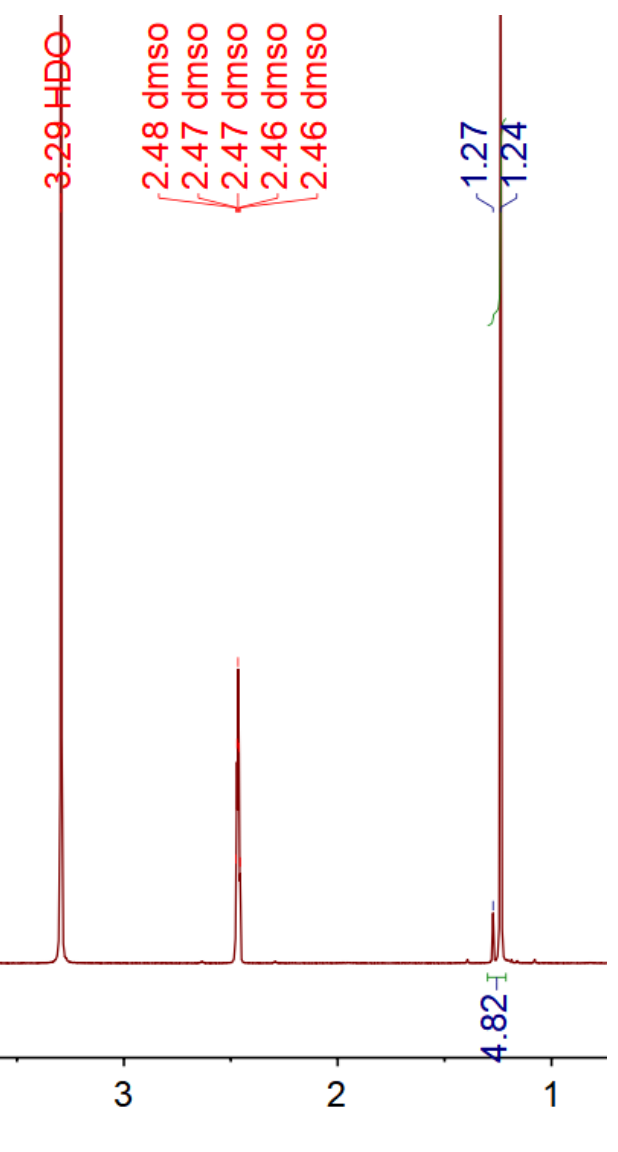

Figure S5. ${ }^{1} \mathrm{H}$ NMR of Tetra(4-dihydroxyborylphenyl)methane in DMSO-d 6 
IV. Covalent Organic Framework Structures

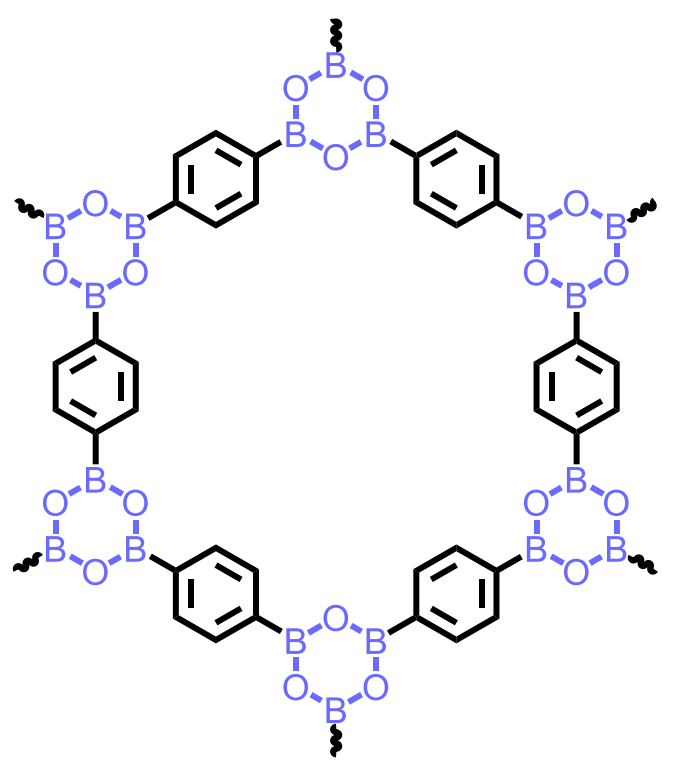

Figure S6. Structure of Ph-COF

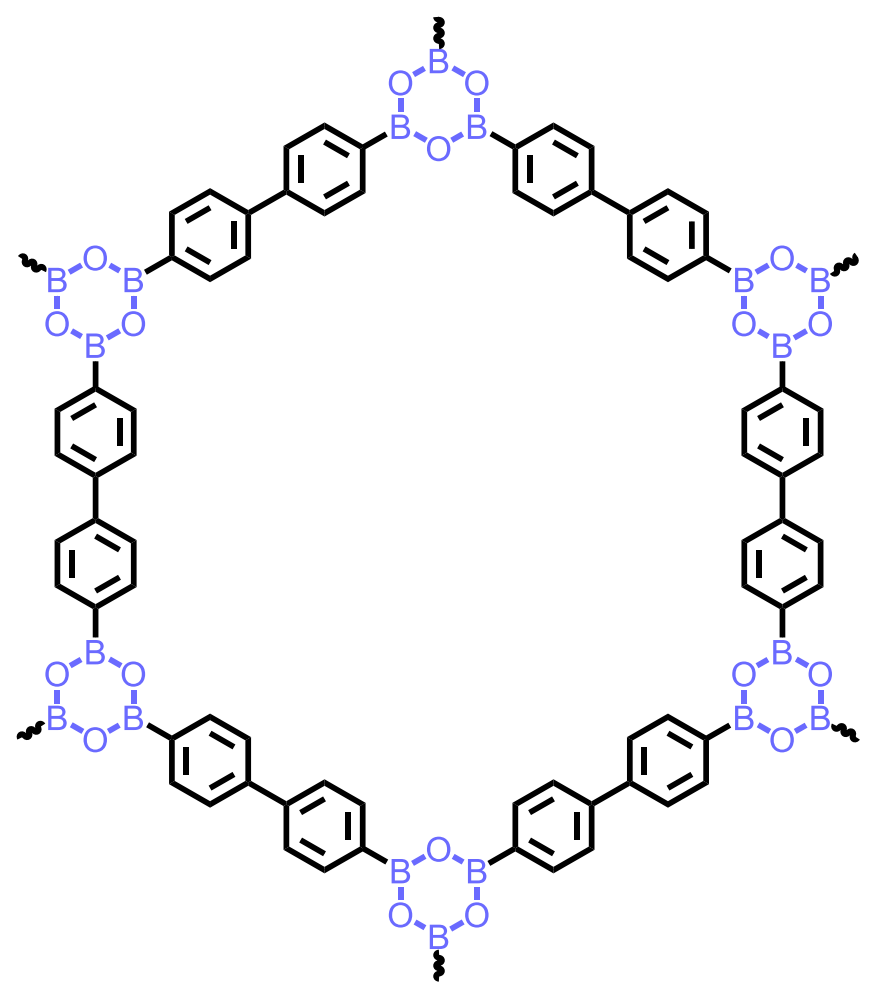

Figure S7. Structure of BPh-COF 


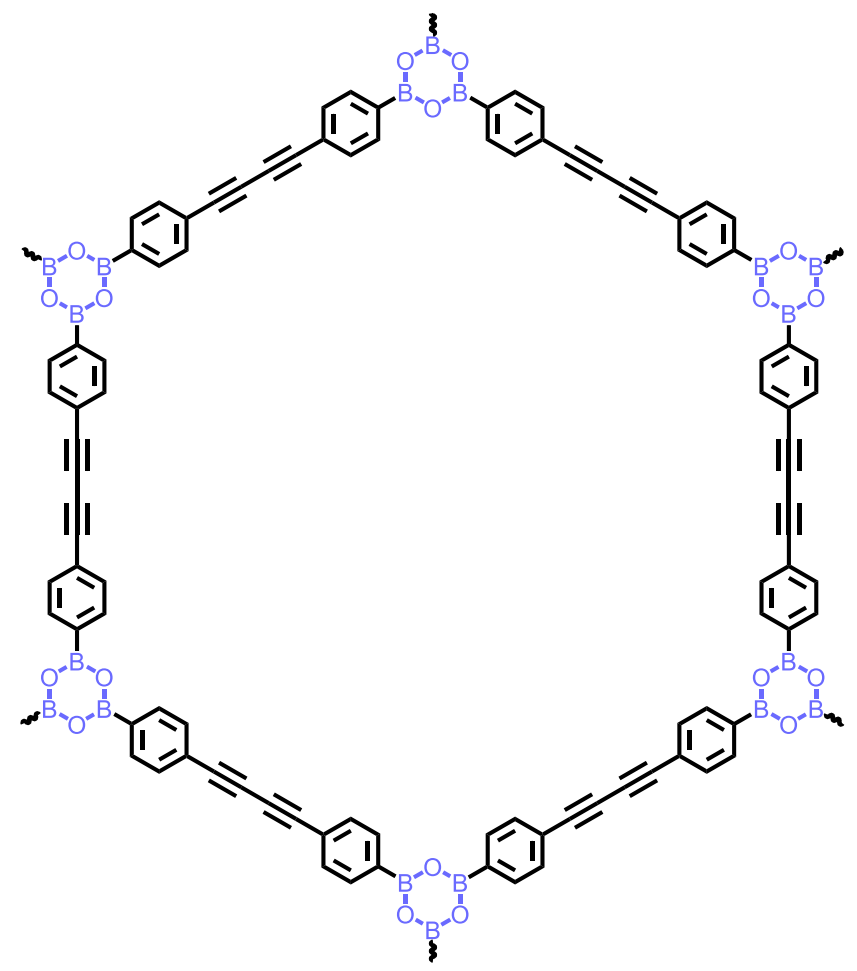

Figure S8. Structure of DBD-COF

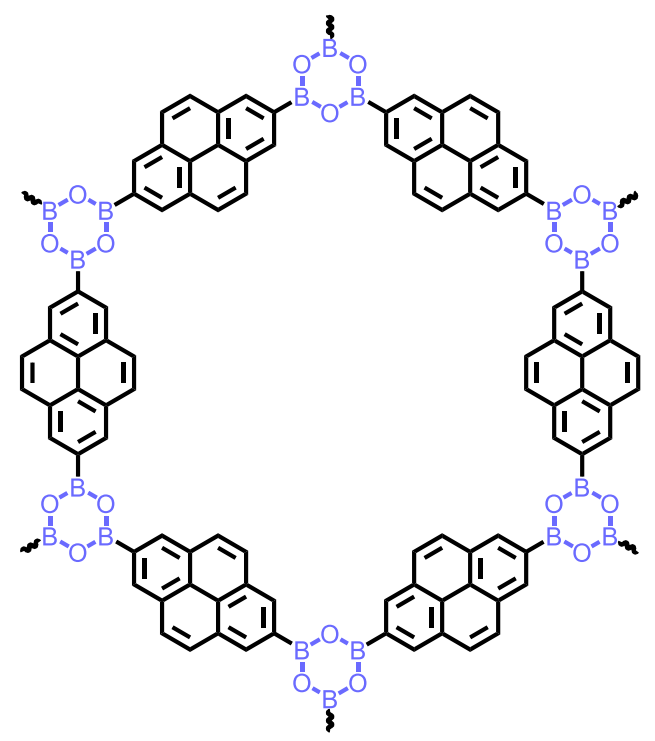

Figure S9. Structure of Py-COF

15 


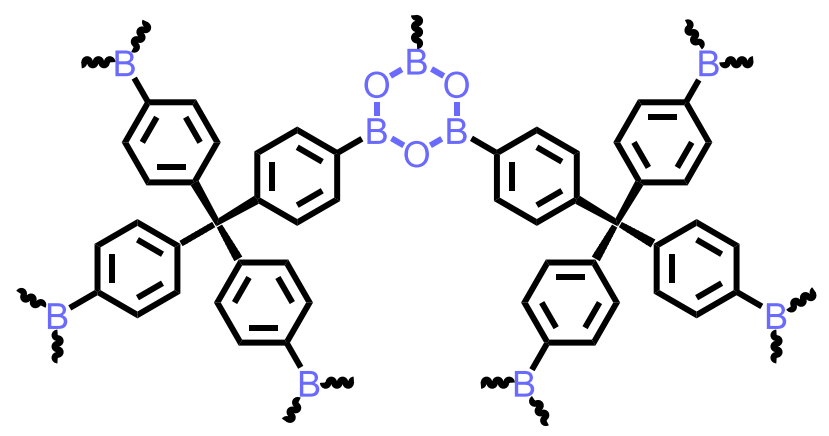

Figure S10. Structure of TMPh-COF 


\section{Synthesis of Covalent Organic Frameworks}

\section{Scheme S4. Synthesis of Ph-COF}

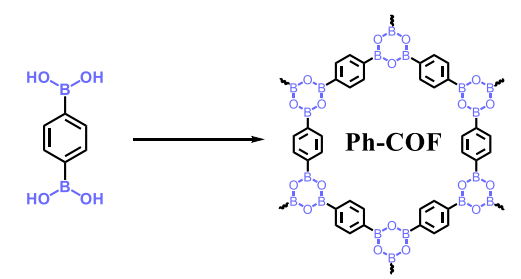

Ph-COF. Phenylenebisboronic acid (PBBA) $(10 \mathrm{mM}, 33.2 \mathrm{mg}) *$ was dissolved in either a mixture of 4:1 1,4-dioxane:mesitylene $(20 \mathrm{~mL})$ when attempting powder synthesis of $\mathbf{P h - C O F}$ or a mixture of $\mathrm{CH}_{3} \mathrm{CN}^{* *}: 1,4$-dioxane:mesitylene $(20 \mathrm{~mL})(50: 40: 10, v / v / v) * * *$ when attempting colloidal syntheses. The PBBA solution was then heated to $70{ }^{\circ} \mathrm{C}$ for 72 hours. During this time, the clear solutions became white and opaque.

*For experiments where PBBA concentrations were varied, all other factors were kept the same.

**For experiments where other cosolvents were tested, those solvents were substituted for the acetonitrile.

***For experiments where the total ratio of solvents were varied, the mixtures varied between $90-0 \mathrm{CH}_{3} \mathrm{CN}$ vol \% with the rest of the solvent being constructed from 1:4 mesitylene:1,4-dioxane.

Scheme S5. Synthesis of BPh-COF

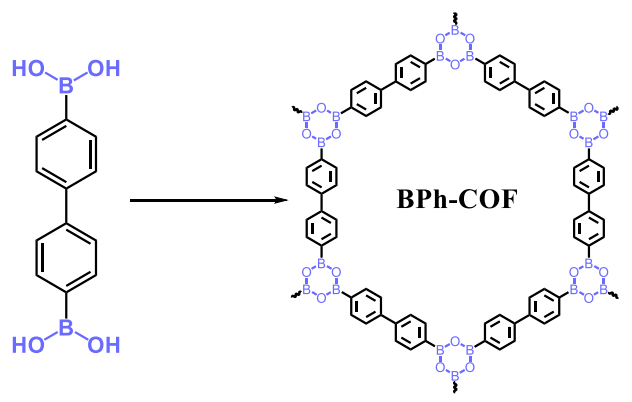

BPh-COF. Biphenylenebisboronic acid (BBBA) $(10 \mathrm{mM}, 12 \mathrm{mg})$ was dissolved in a mixture of $\mathrm{CH}_{3} \mathrm{CN}$ :1,4-dioxane:mesitylene $(5 \mathrm{~mL})(50: 40: 10, v / v / v)$ when attempting colloidal syntheses. The BBBA solution was then heated to $70{ }^{\circ} \mathrm{C}$ for 72 hours. During this time, the clear solutions became white and opaque. 


\section{Scheme S6. Synthesis of DBD-COF}

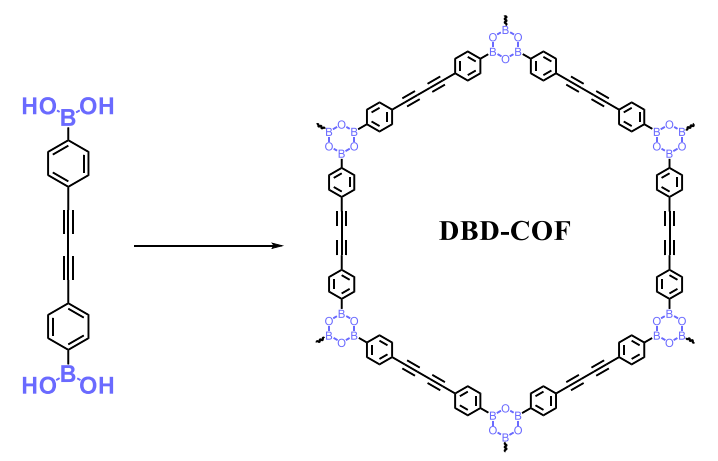

DBD-COF. Diphenylbutadiynbisboronic acid (DBD-BA) $(10 \mathrm{mM}, 14.5 \mathrm{mg})$ was dissolved in a mixture of $\mathrm{CH}_{3} \mathrm{CN}: 1,4-$ dioxane:mesitylene $(5 \mathrm{~mL})(50: 40: 10, v / v / v)$ when attempting colloidal syntheses. The DBDBA solution was then heated to $70{ }^{\circ} \mathrm{C}$ for 72 hours. During this time, the clear solutions became white and opaque.

\section{Scheme S7. Synthesis of Py-COF}
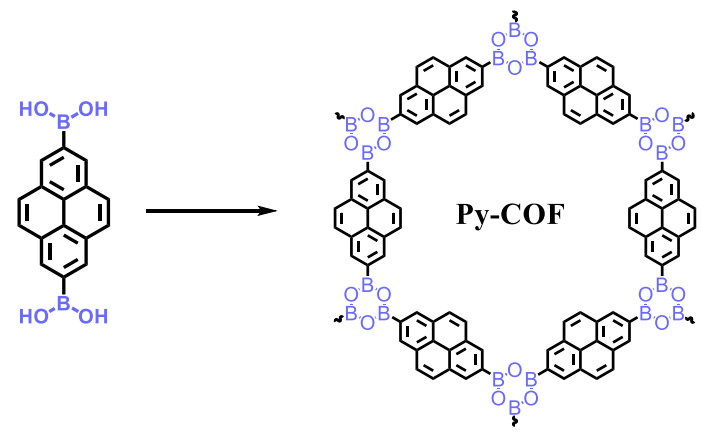

Py-COF. Pyrenebisboronic acid (PyBA) (10 mM, $14.5 \mathrm{mg})$ was dissolved in a mixture of $\mathrm{CH}_{3} \mathrm{CN}: 1,4-$ dioxane:mesitylene $(5 \mathrm{~mL})(50: 40: 10, v / v / v)$ when attempting colloidal syntheses. The Py-BA solution was then heated to $70{ }^{\circ} \mathrm{C}$ for 72 hours. During this time, the clear solutions became white and opaque. 


\section{Scheme S8. Synthesis of TMPh-COF}

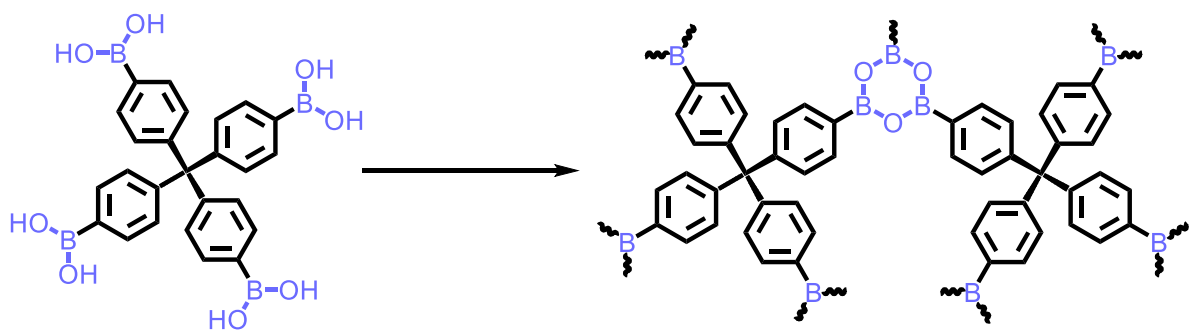

TMPh-COF. Tetrakis(phenlboronic acid)methane (TMPh-BA) $(10 \mathrm{mM}, 24.5 \mathrm{mg})$ was dissolved in a mixture of $\mathrm{CH}_{3} \mathrm{CN}: 1,4-$ dioxane:mesitylene $(5 \mathrm{~mL})(50: 40: 10, v / v / v)$ when attempting colloidal syntheses. The TMPh-BA solution was then heated to $70{ }^{\circ} \mathrm{C}$ for 72 hours. During this time, the clear solutions became white and opaque. 
VI. Fourier-Transform Infrared Spectroscopy of Covalent Organic Frameworks

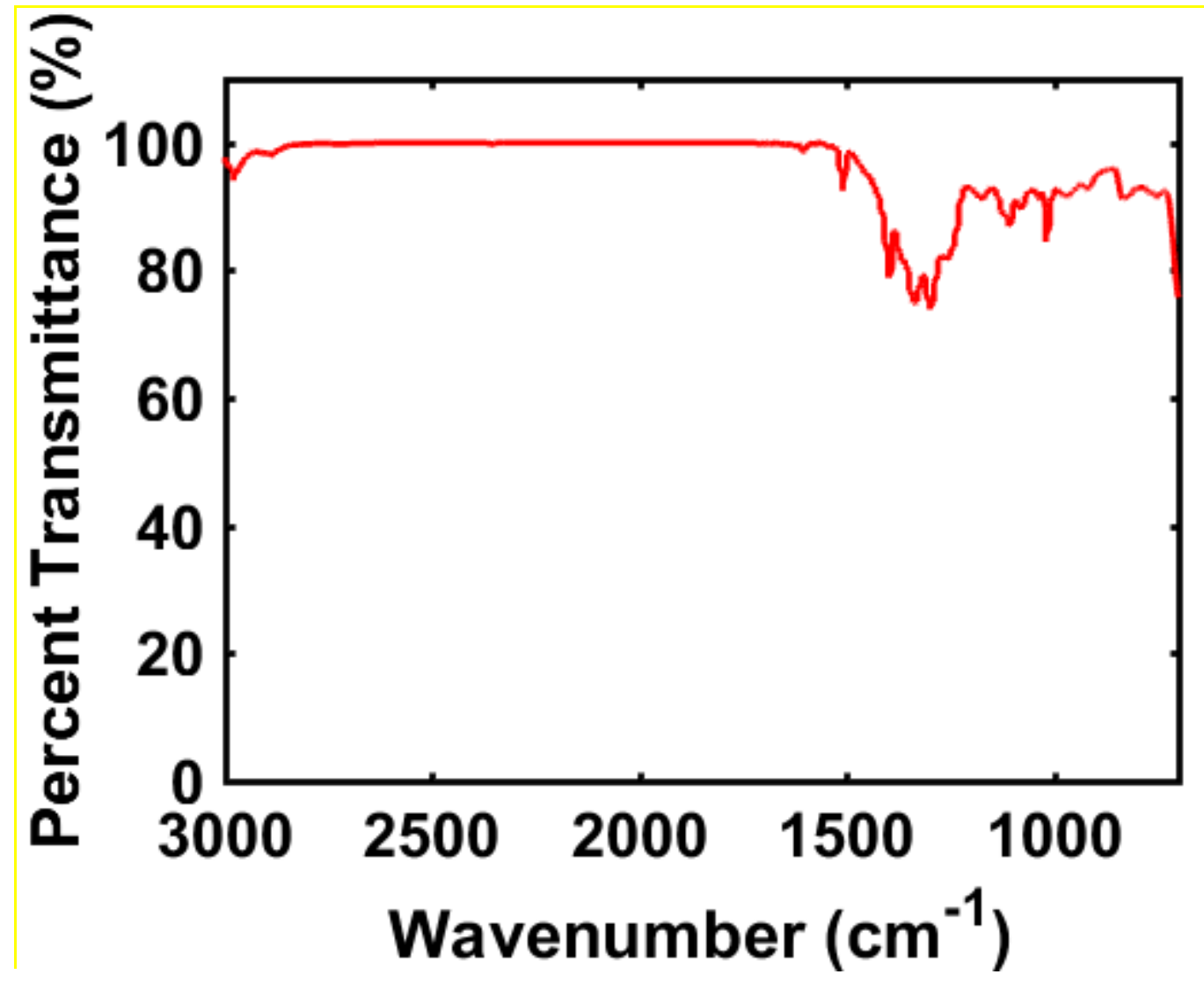

Figure 11. FT-IR of Ph-COF Powder 


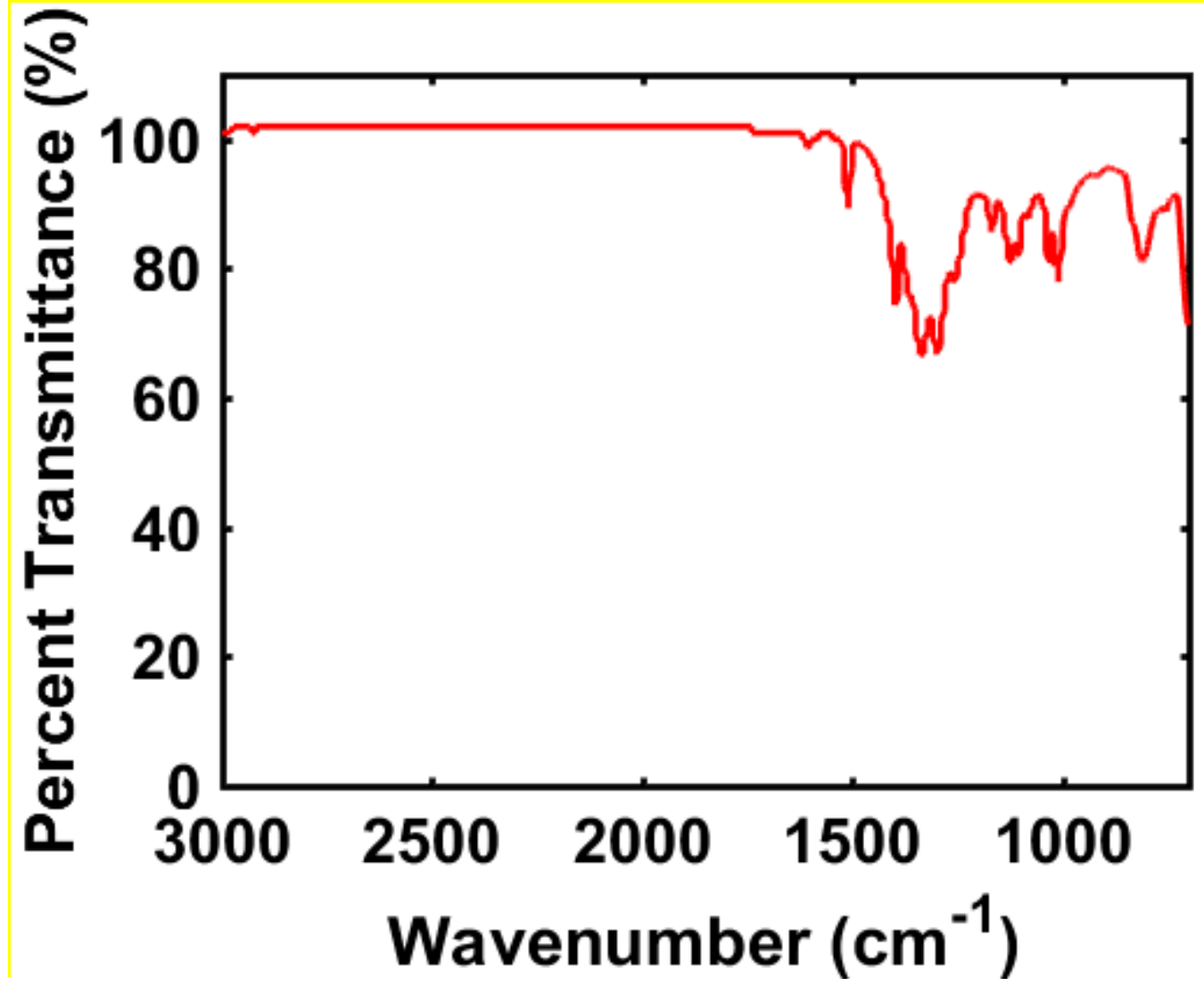

Figure 12. FT-IR of Ph-COF Colloids 


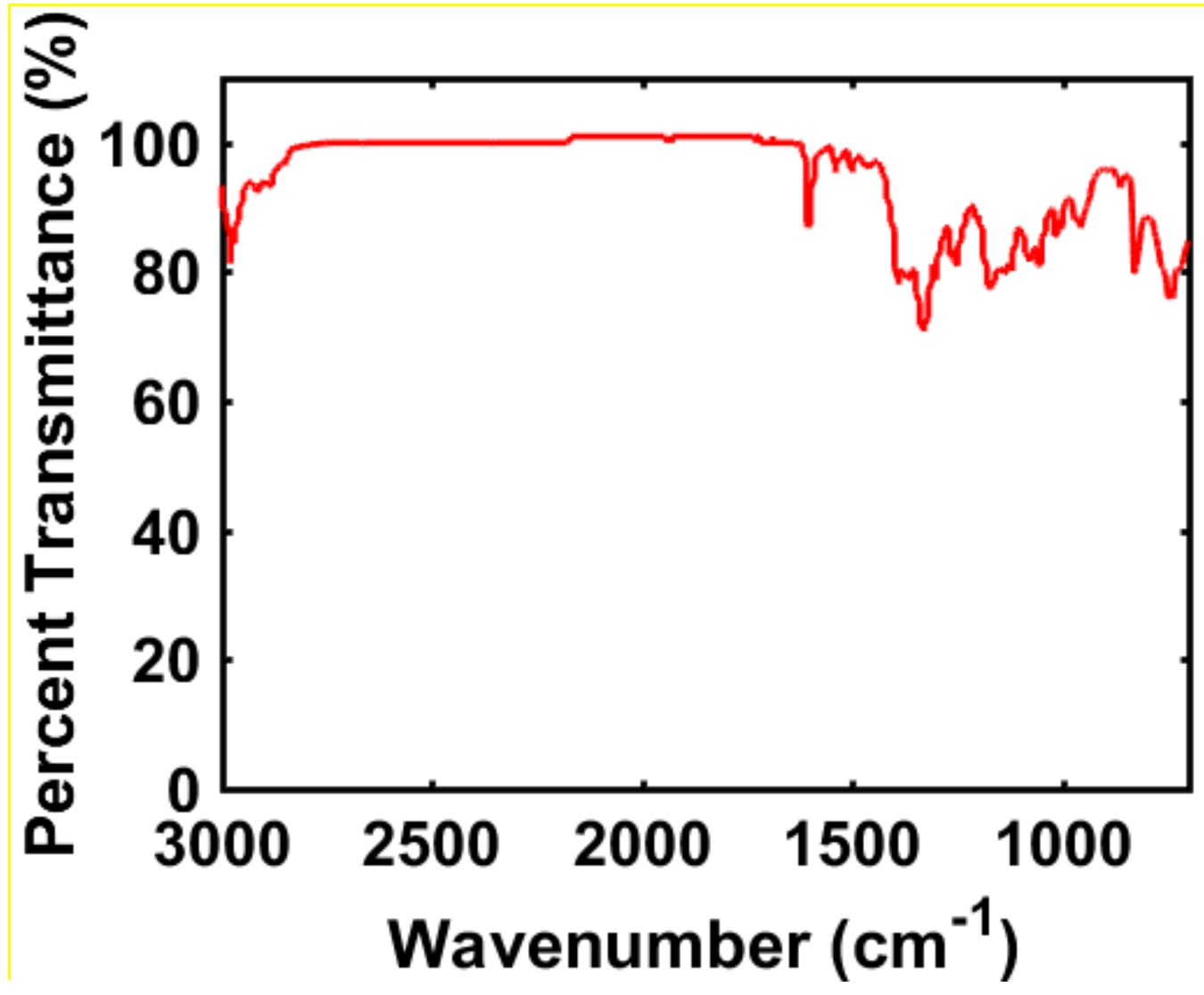

Figure 13. FT-IR of BPh-COF Colloids 


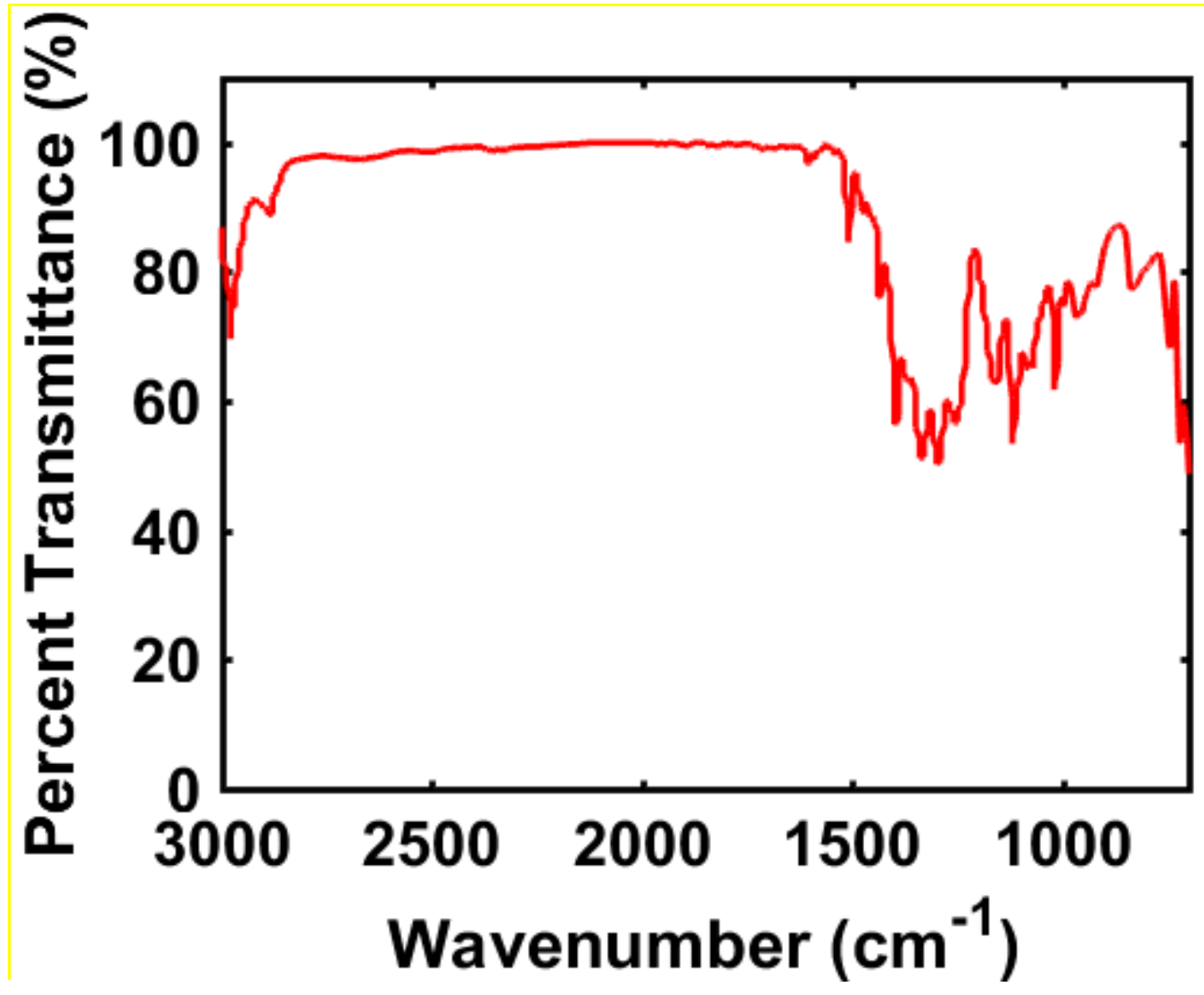

Figure 14. FT-IR of DBD-COF Colloids 


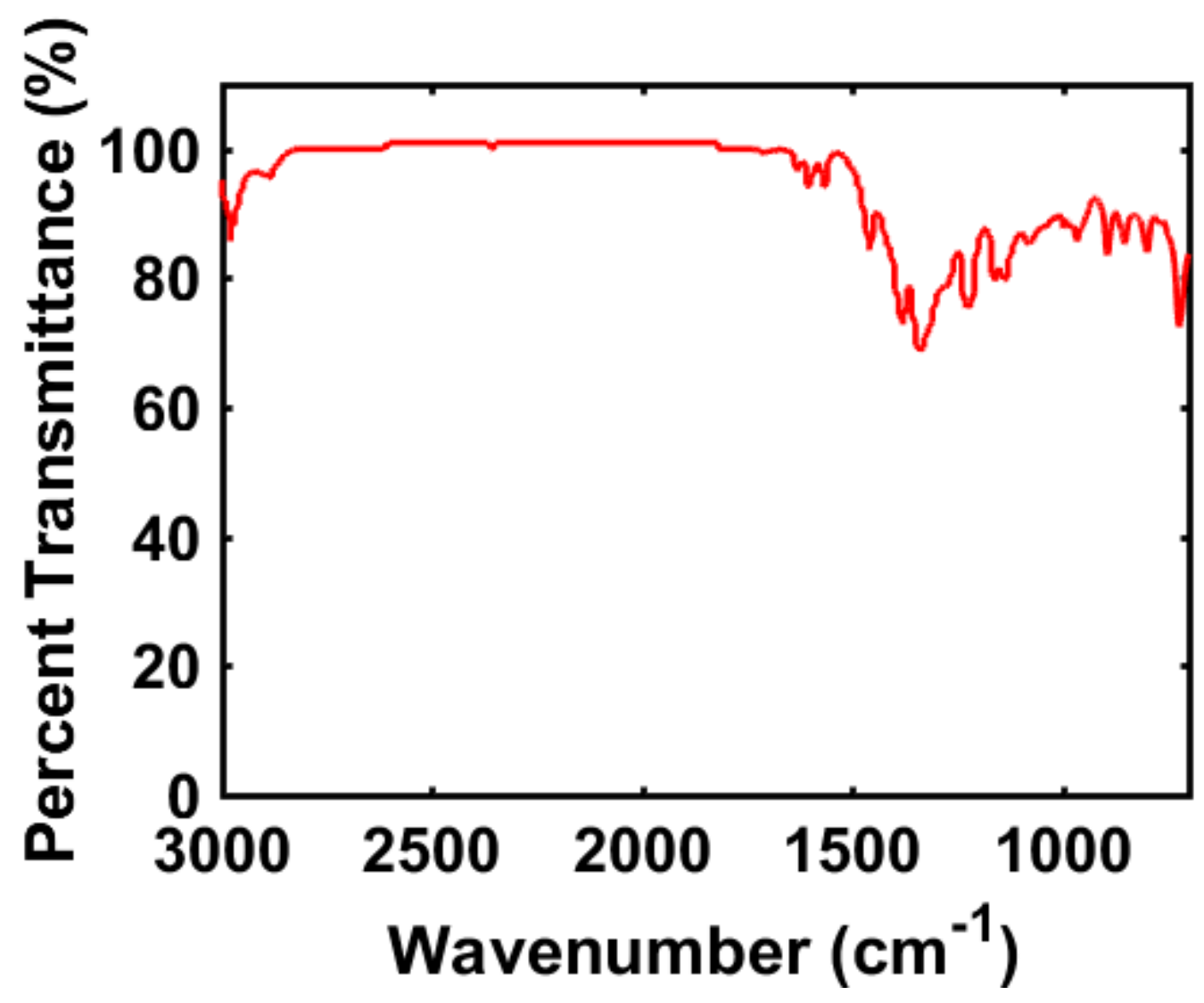

Figure 15. FT-IR of Py-COF Colloids 


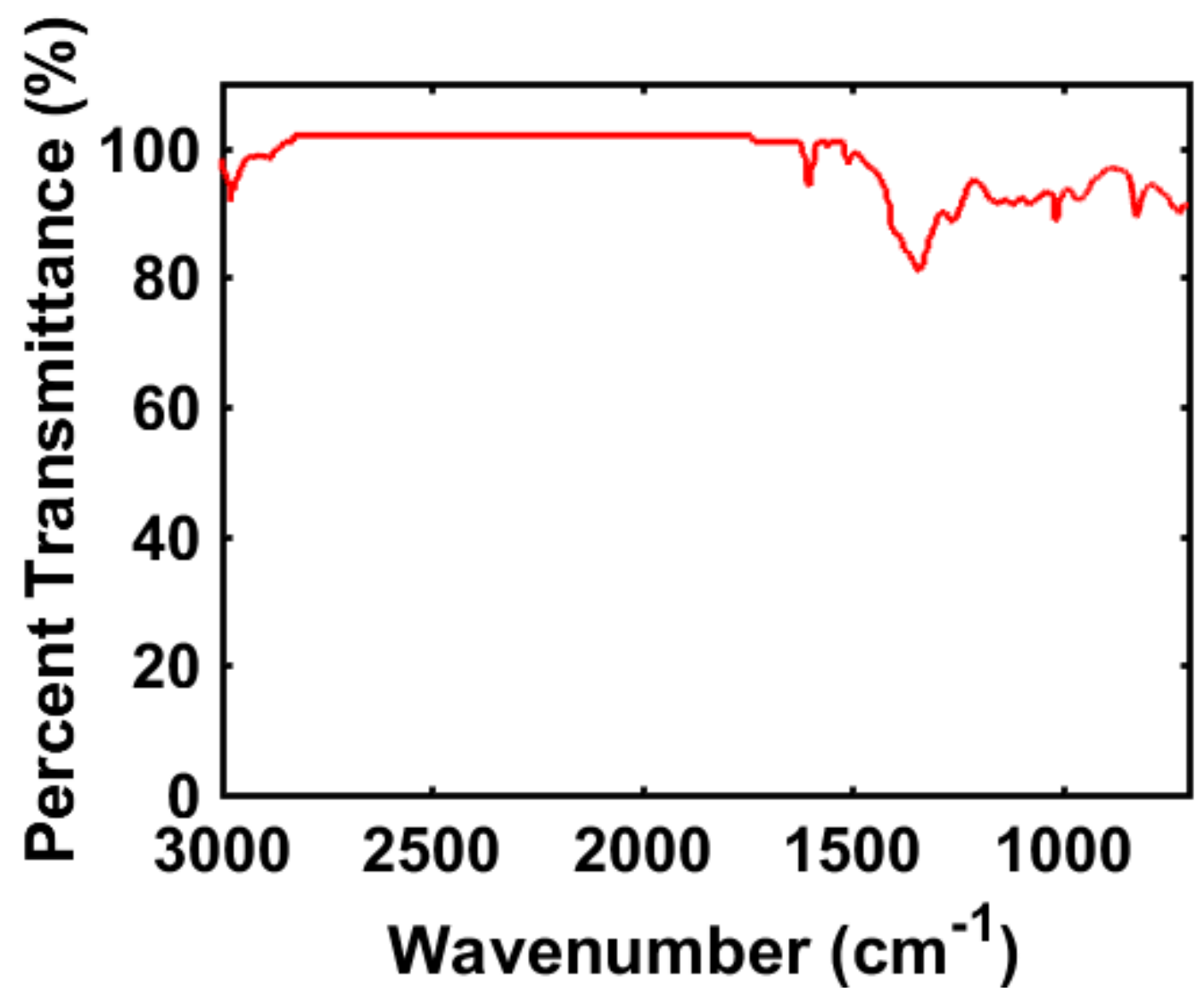

Figure 16. FT-IR of TMPh-COF Colloids 
VII. Dynamic Light Scattering of Covalent Organic Frameworks

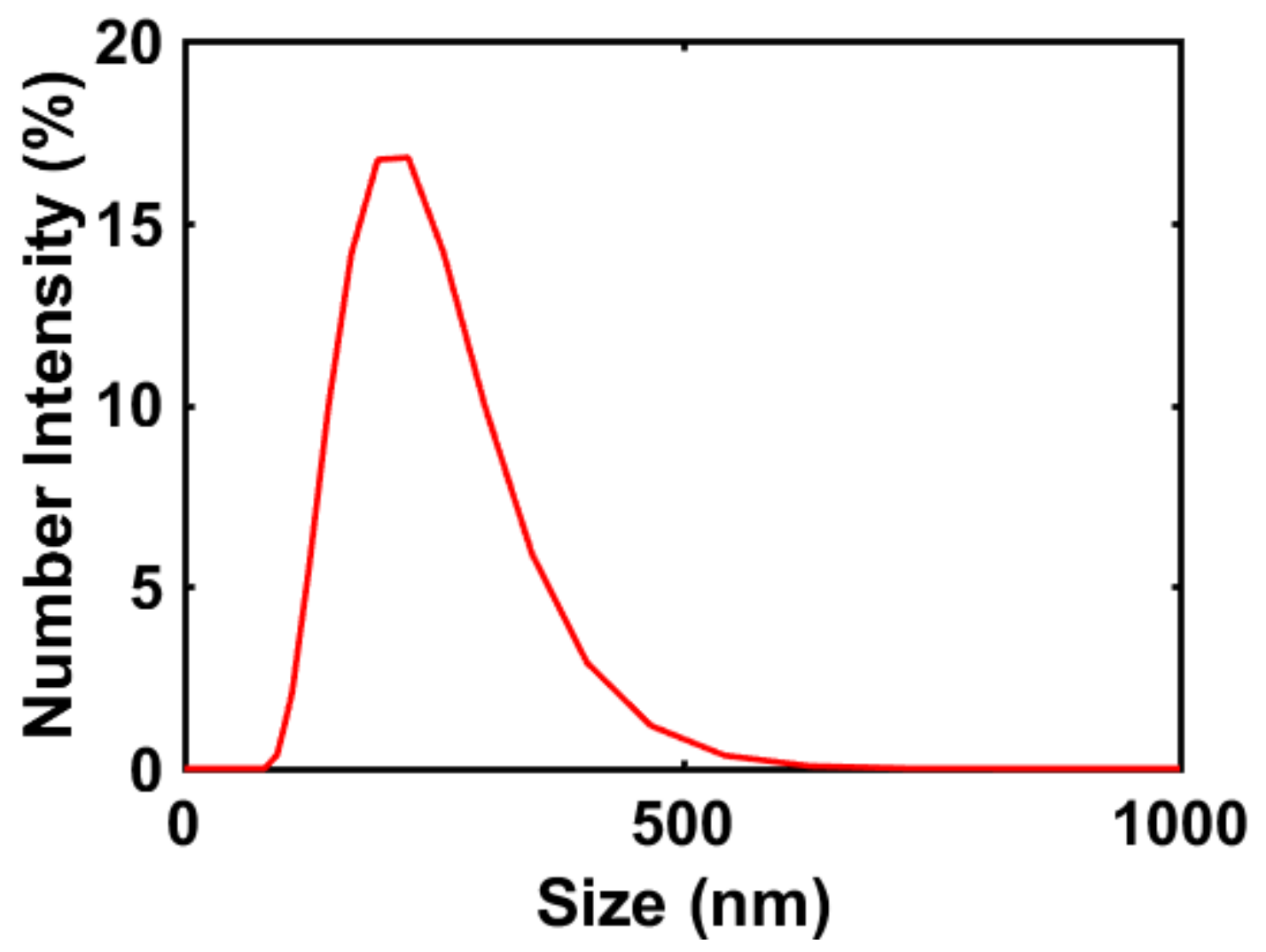

Figure 17. Dynamic light scattering number distribution of $\mathbf{P h - C O F}$ 


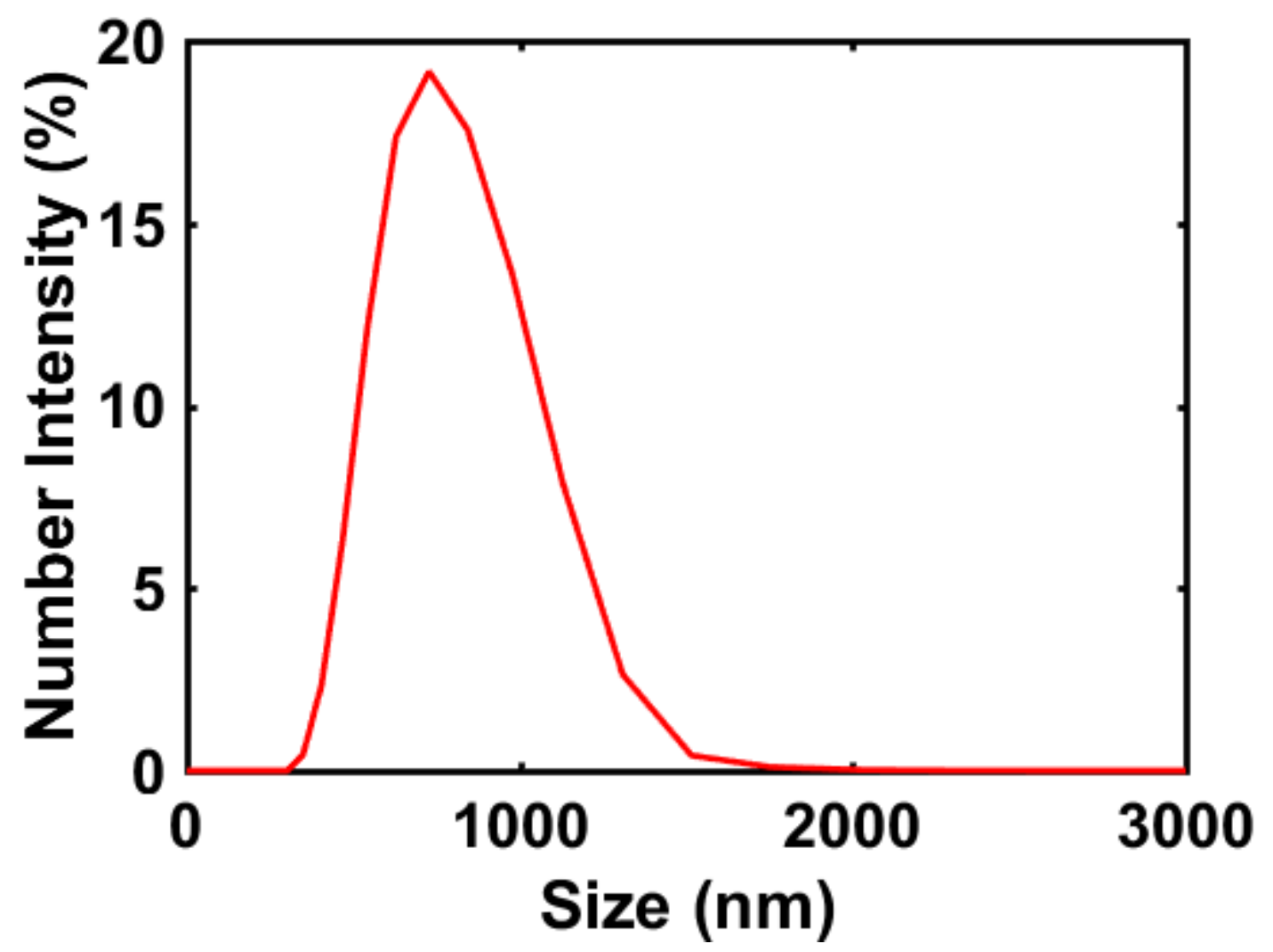

Figure 18. Dynamic light scattering number distribution of BPh-COF 


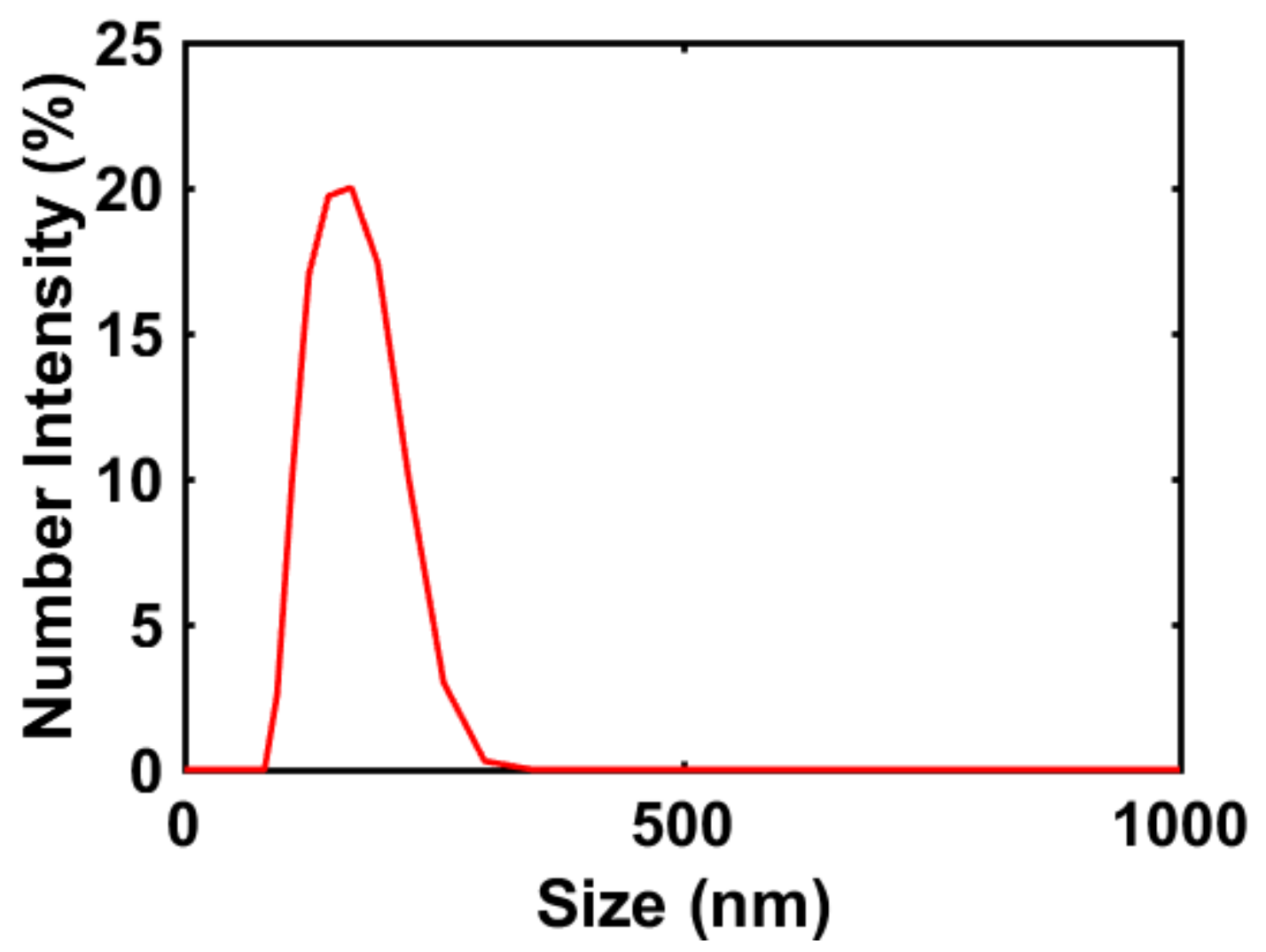

Figure 19. Dynamic light scattering number distribution of DBD-COF 


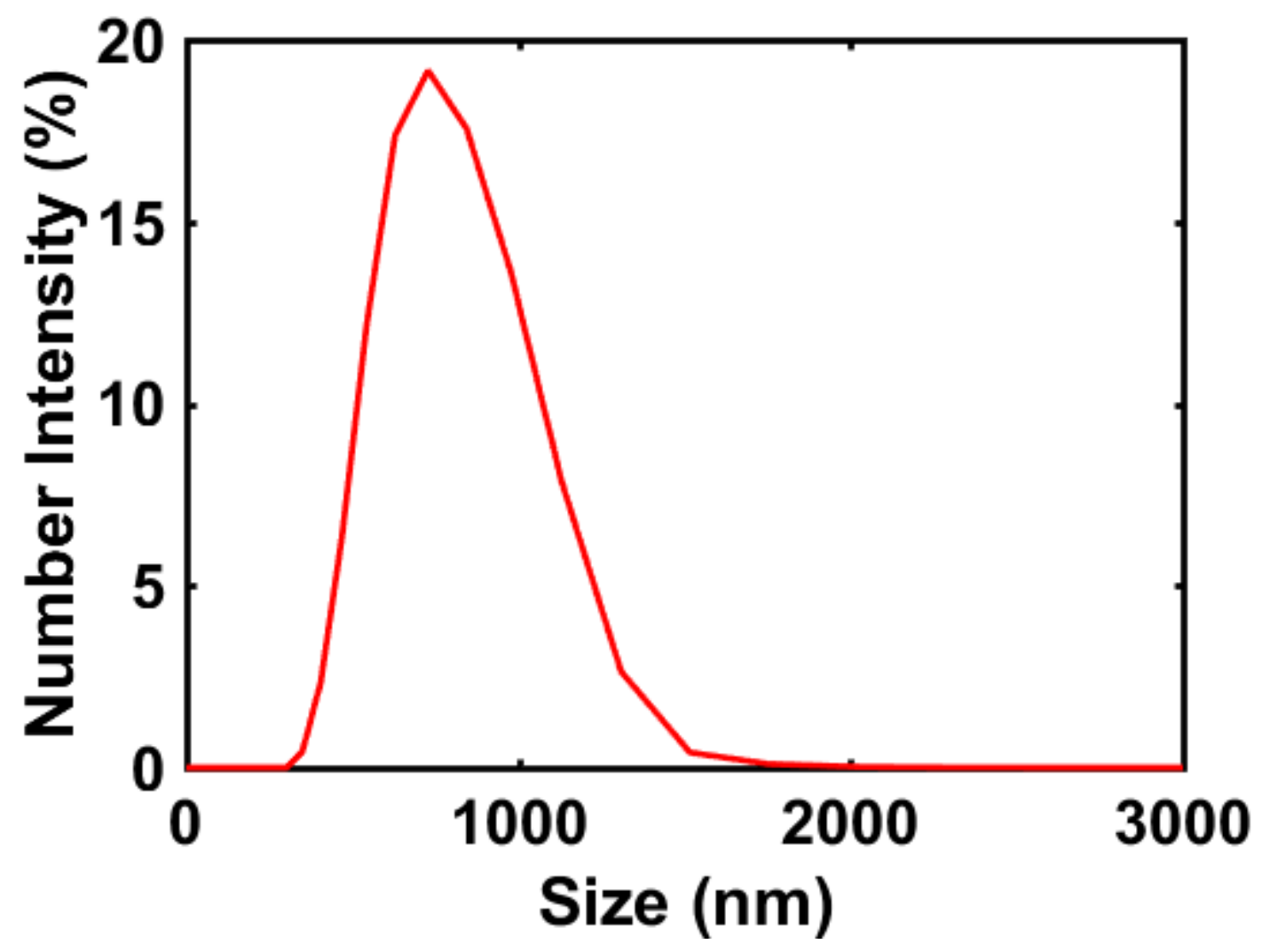

Figure 20. Dynamic light scattering number distribution of Py-COF 


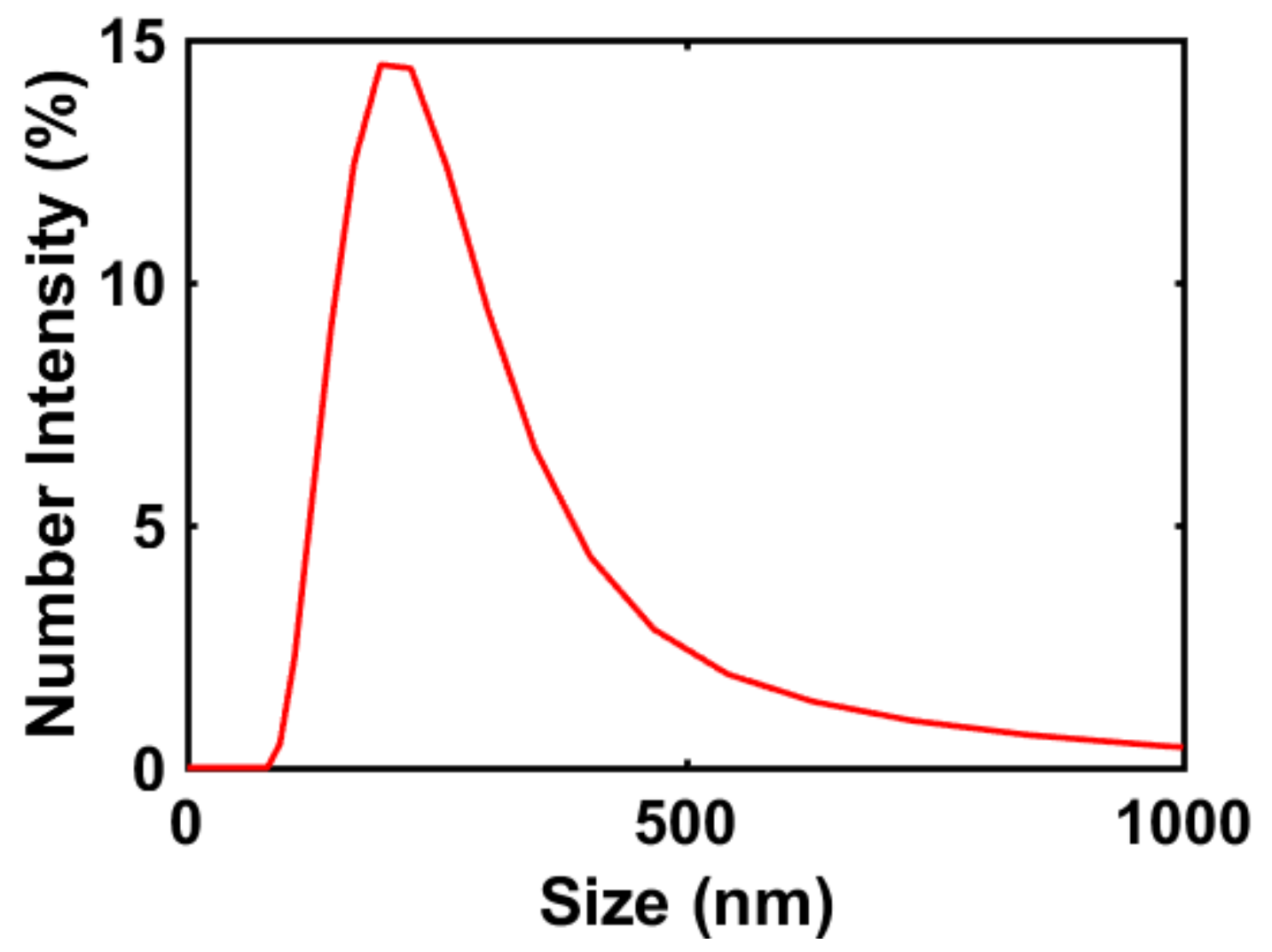

Figure 21. Dynamic light scattering number distribution of TMPh-COF 
VIII. X-ray Diffraction of Collected COF Powders

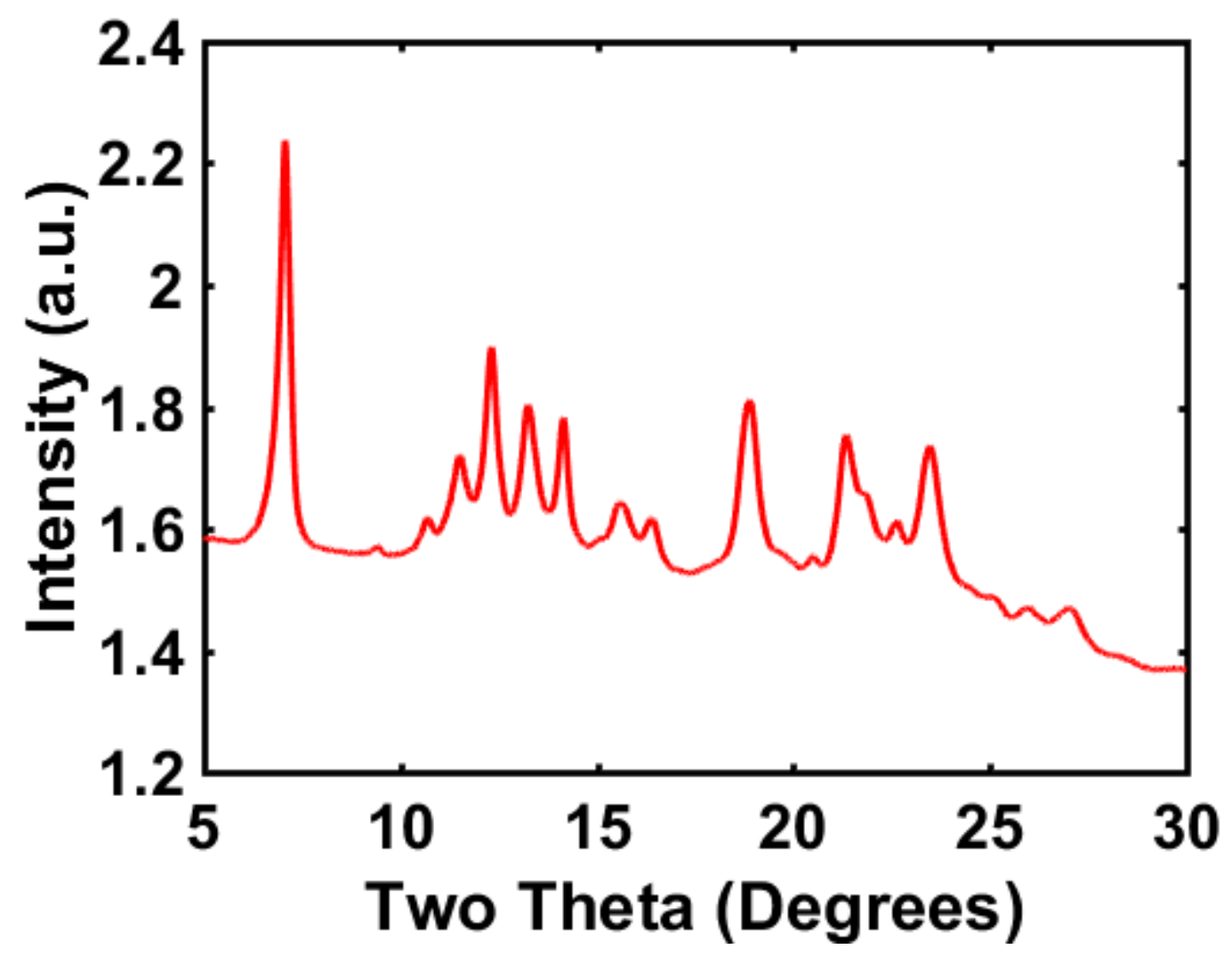

Figure 22. X-ray diffraction of wet Ph-COF 
IX. COF Structural Models

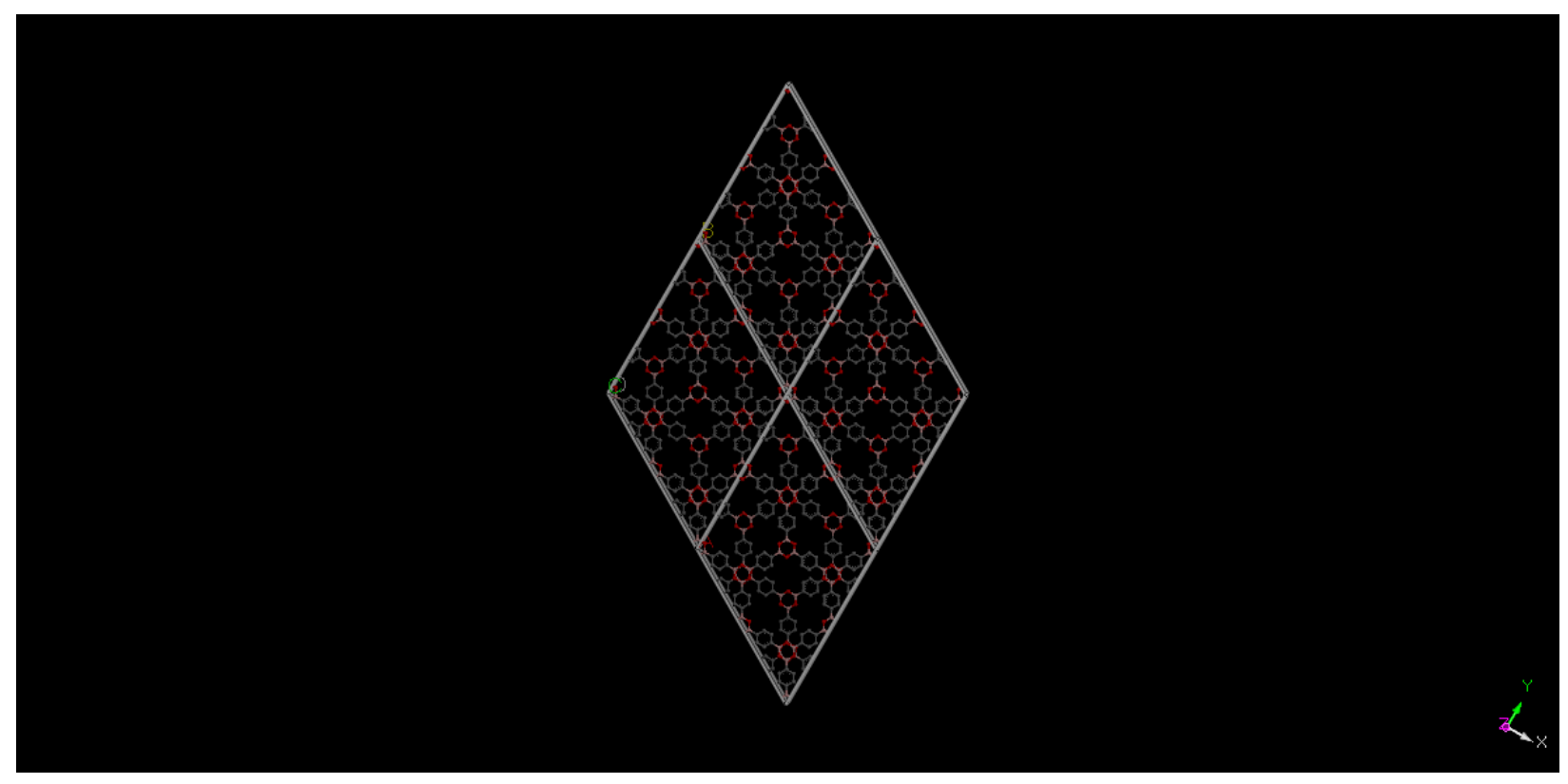

Figure 23. Structure of $\mathbf{P h - C O F}$

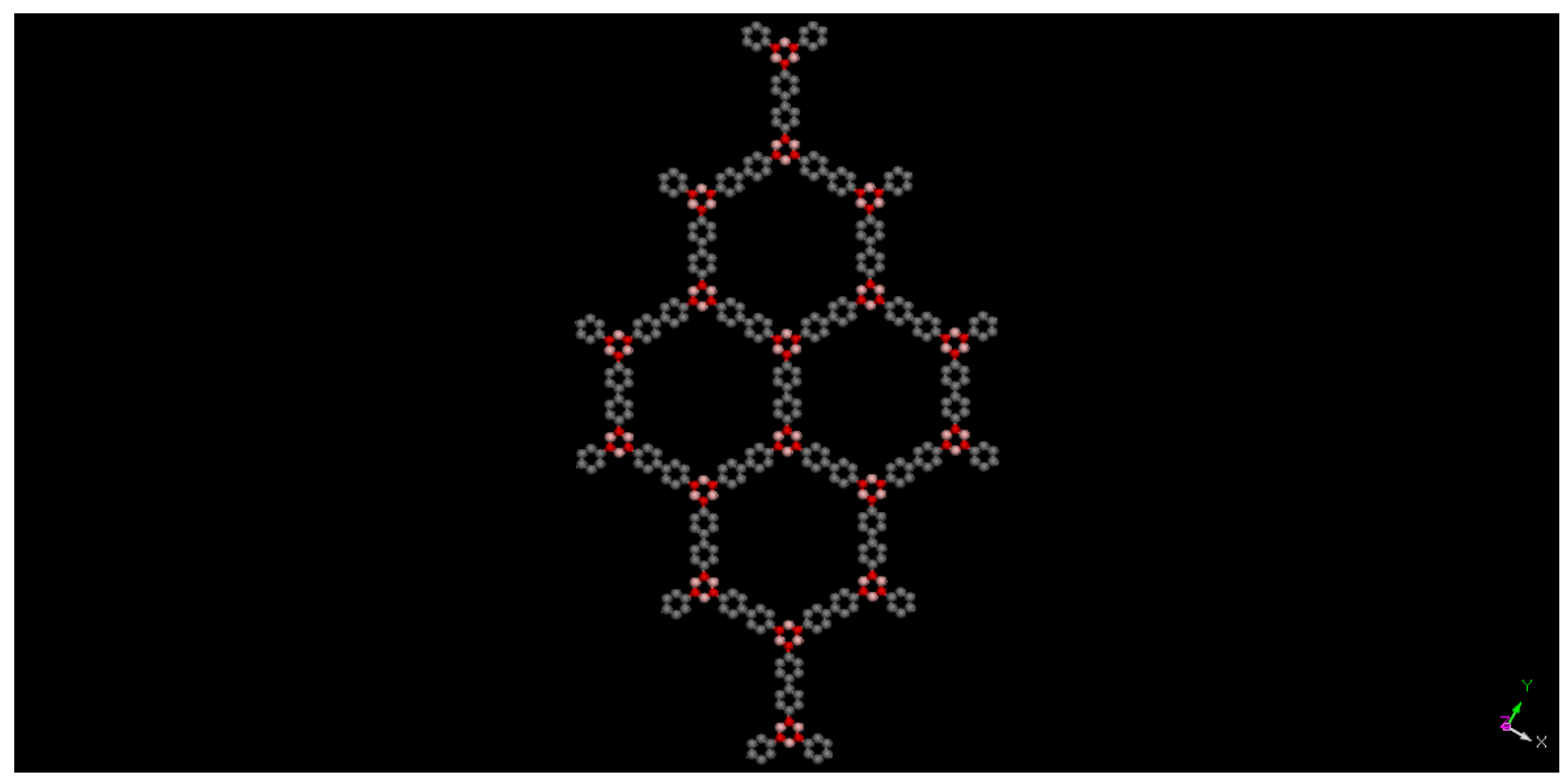

Figure 24. Structure of BPh-COF 


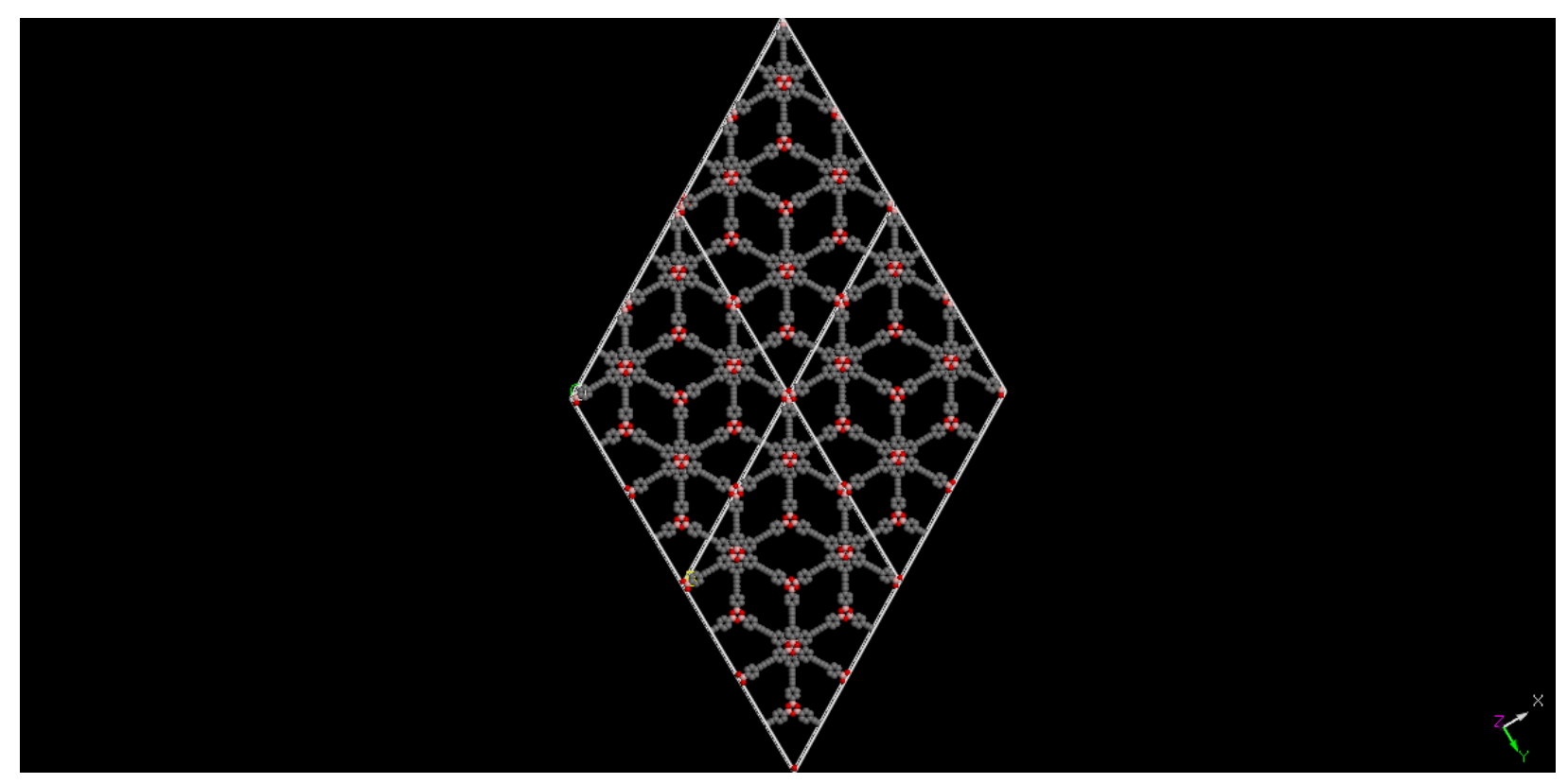

Figure 25. Structure of DBD-COF

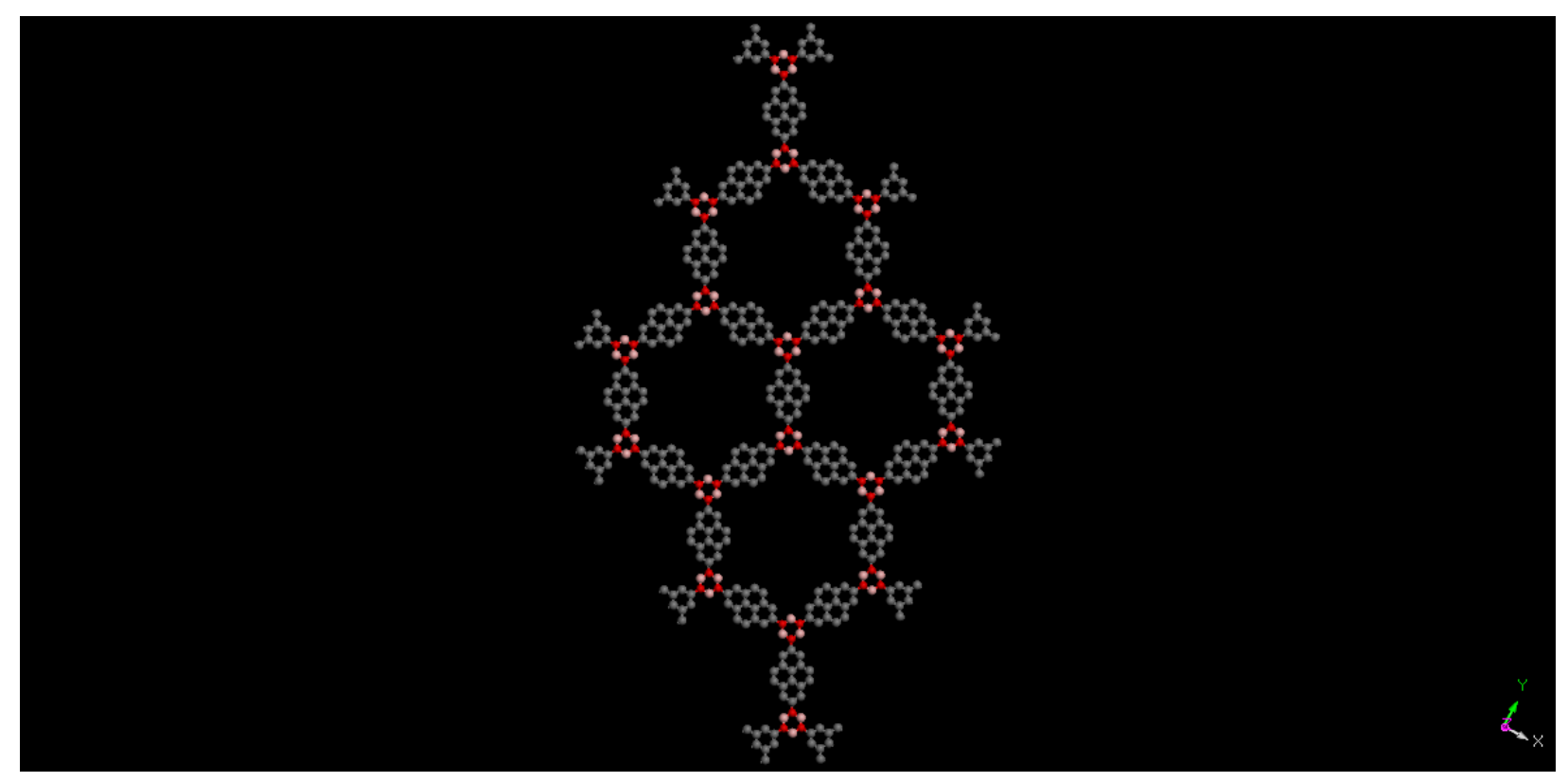

Figure 26. Structure of Py-COF 


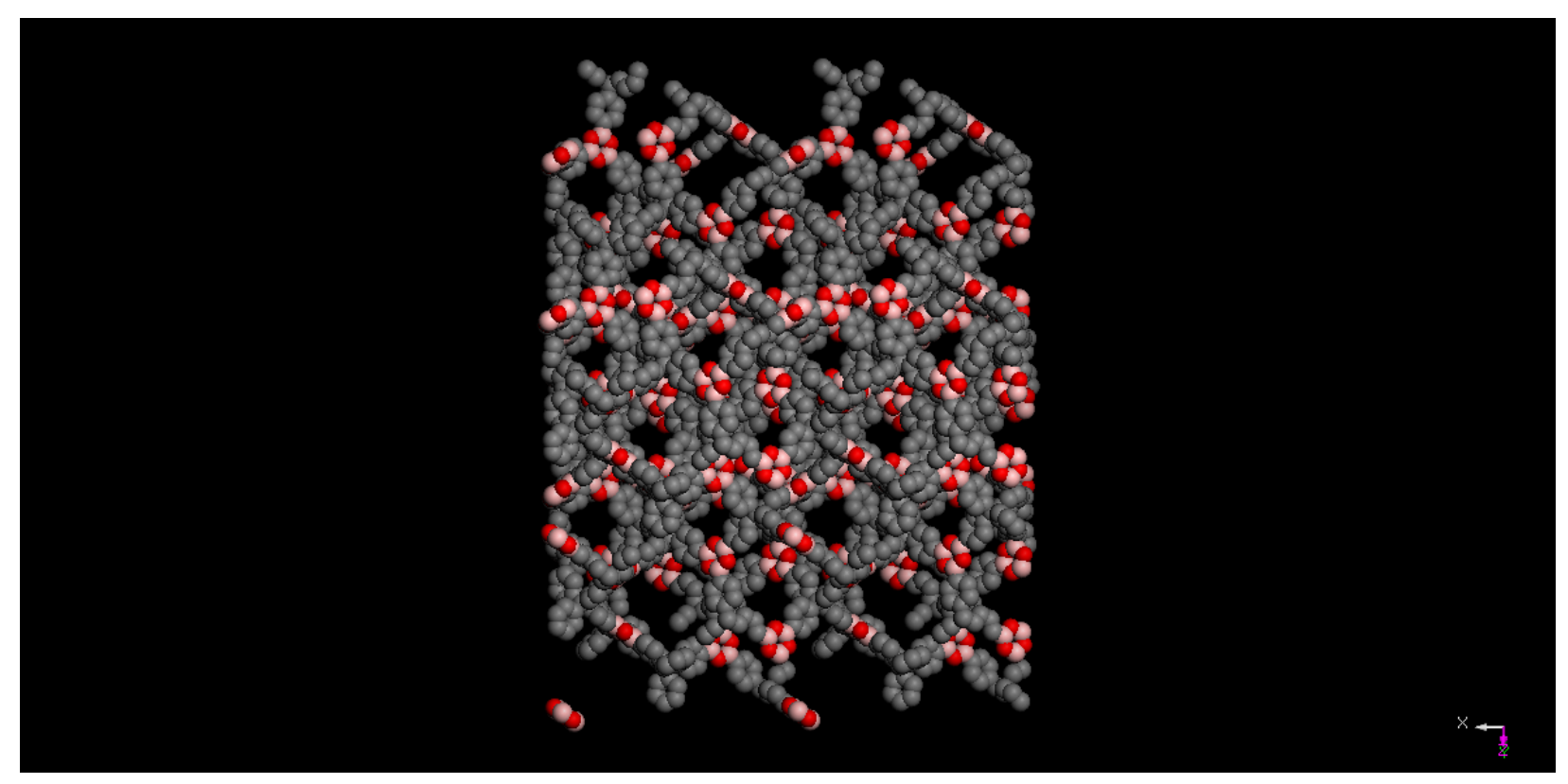

Figure 27. Structure of TMPh-COF

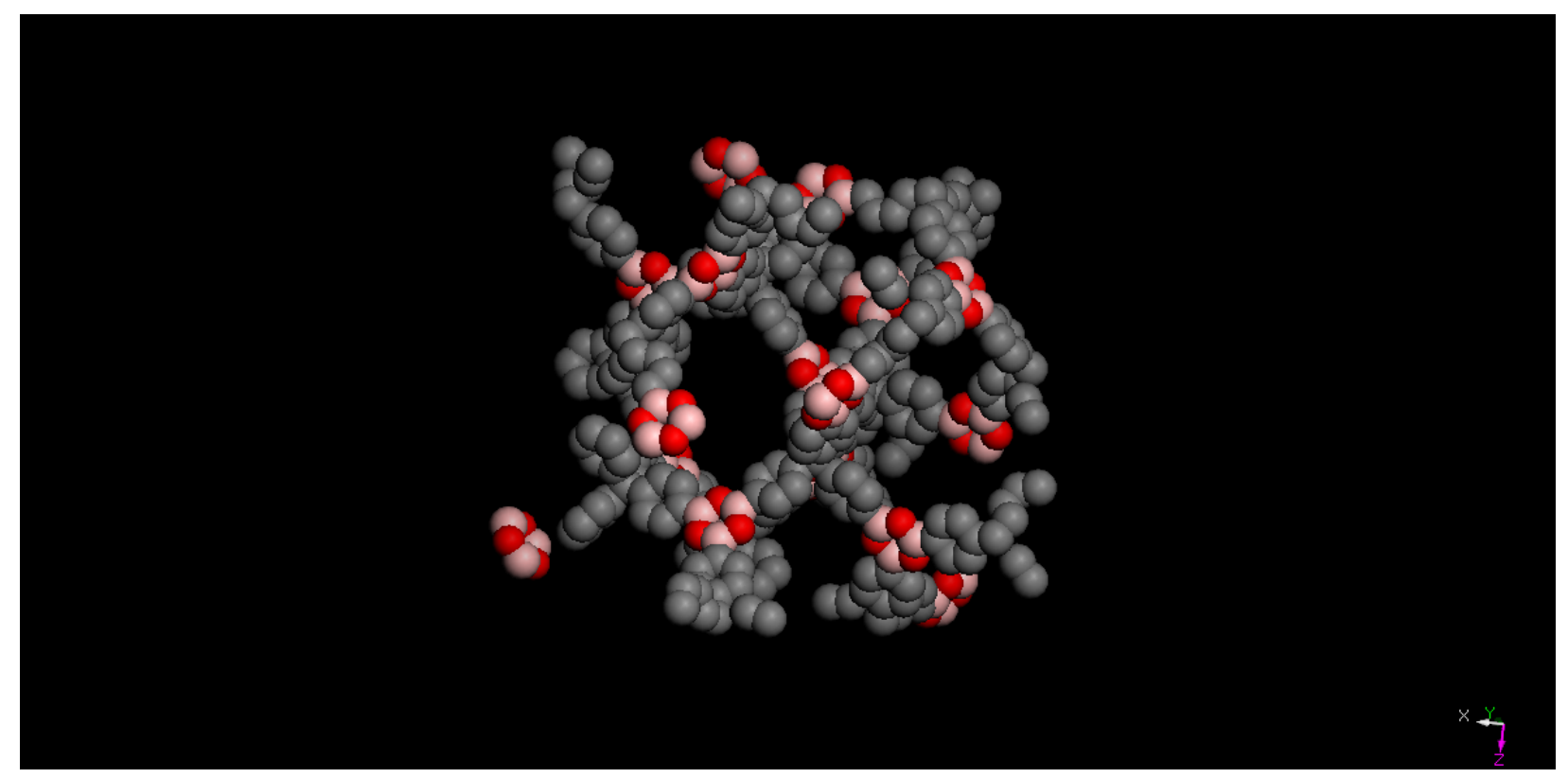

Figure 28. Structure of TMPh-COF - Zoomed in 


\section{COF Modeled Diffraction Patterns}

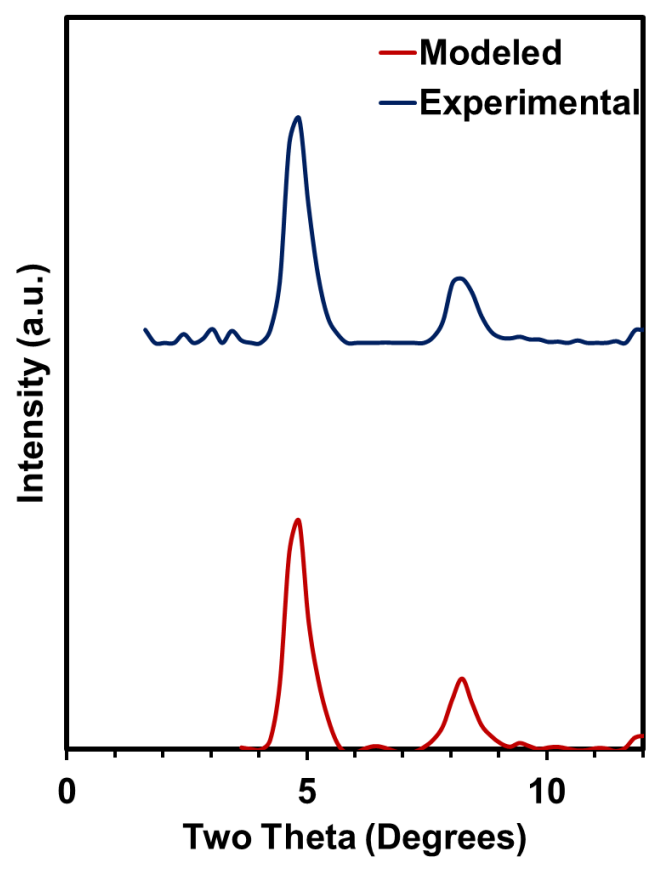

Figure S29. BPh-COF Diffraction Patterns

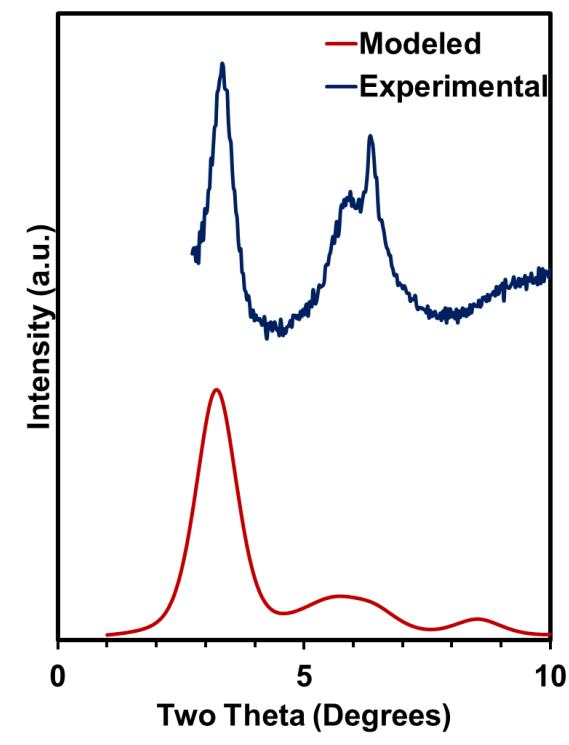

Figure S30. DBD-COF Diffraction Patterns 


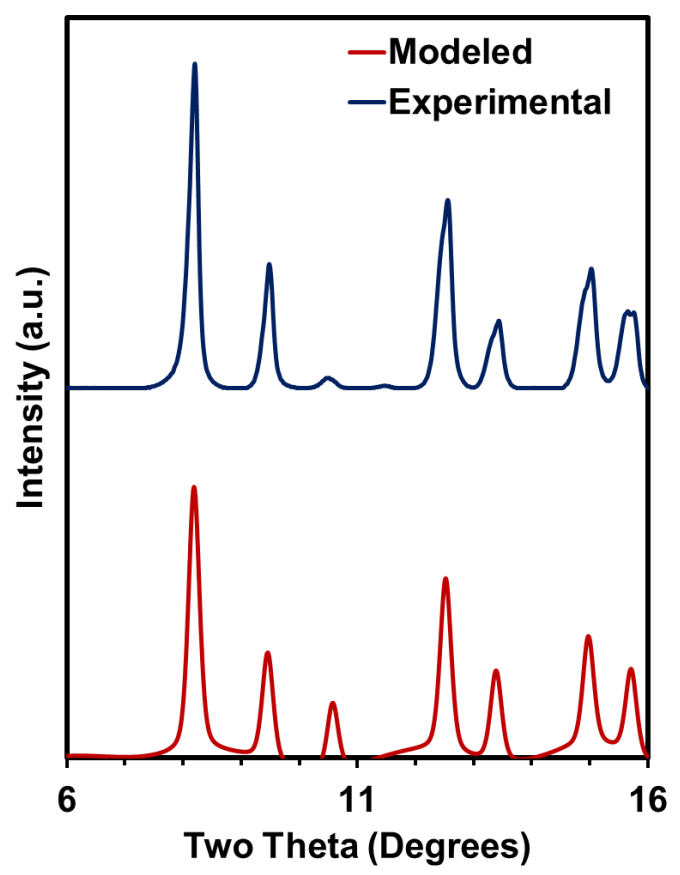

Figure S31. TMPh-COF Diffraction Patterns 


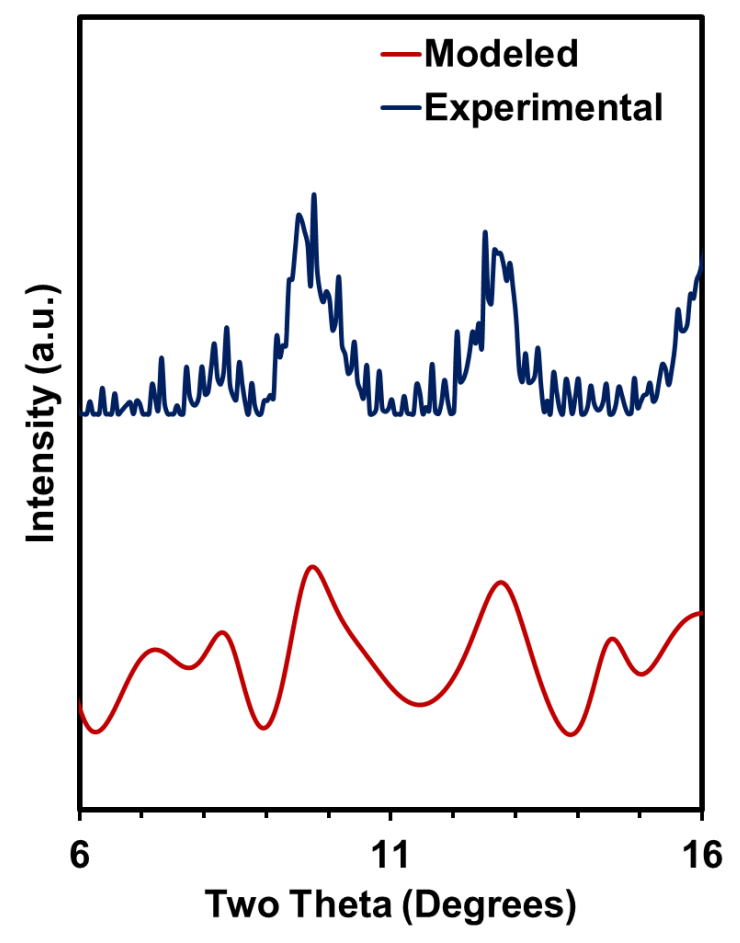

Figure S32. Py-COF Diffraction Patterns 


\section{Additional COF Fluorescence}
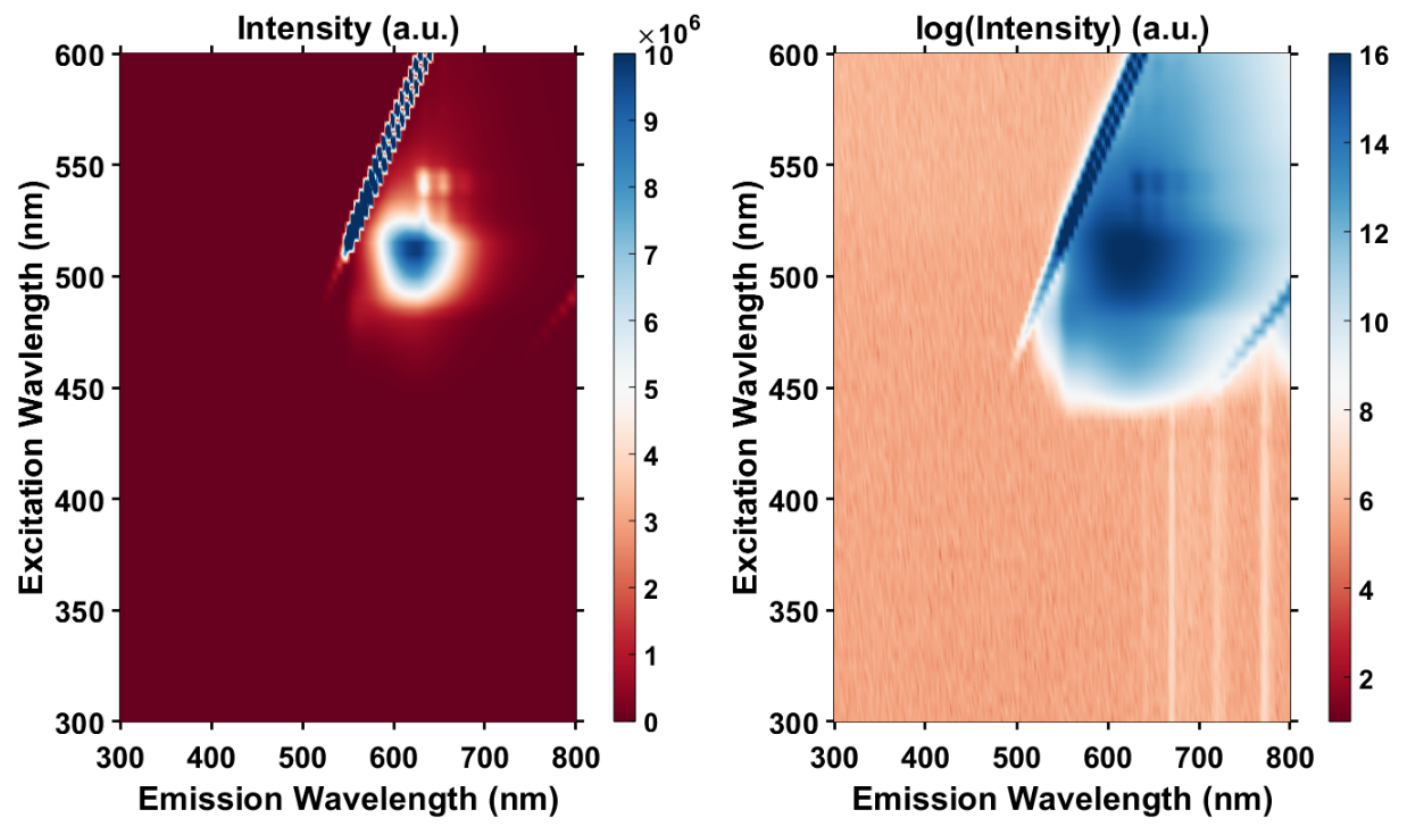

Figure S33. Excitation-Emission Matrix Spectroscopy of Ph-COF
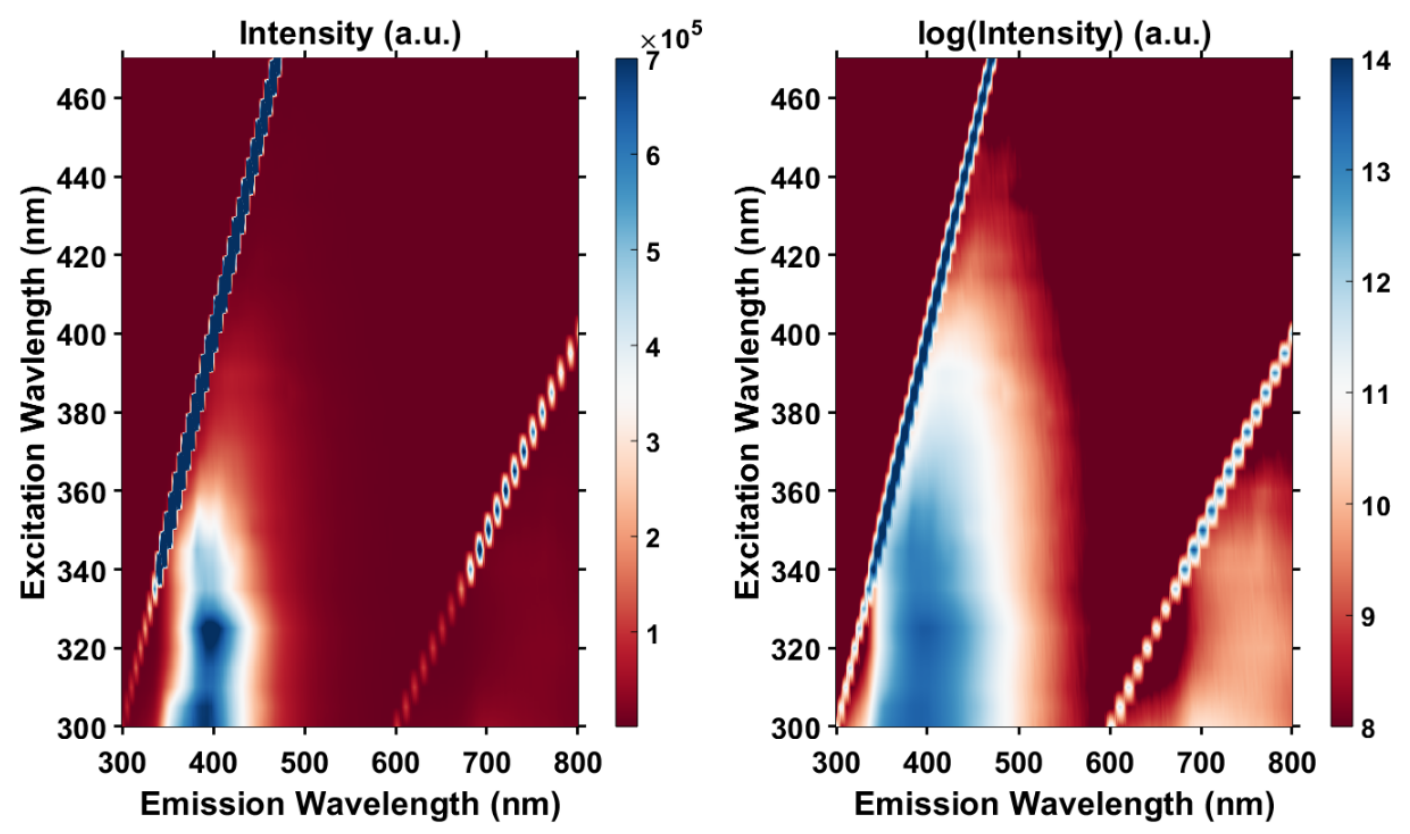

Figure S34. Excitation-Emission Matrix Spectroscopy of BPh-COF 
XII. Additional Transmission Electron Microscopy Images

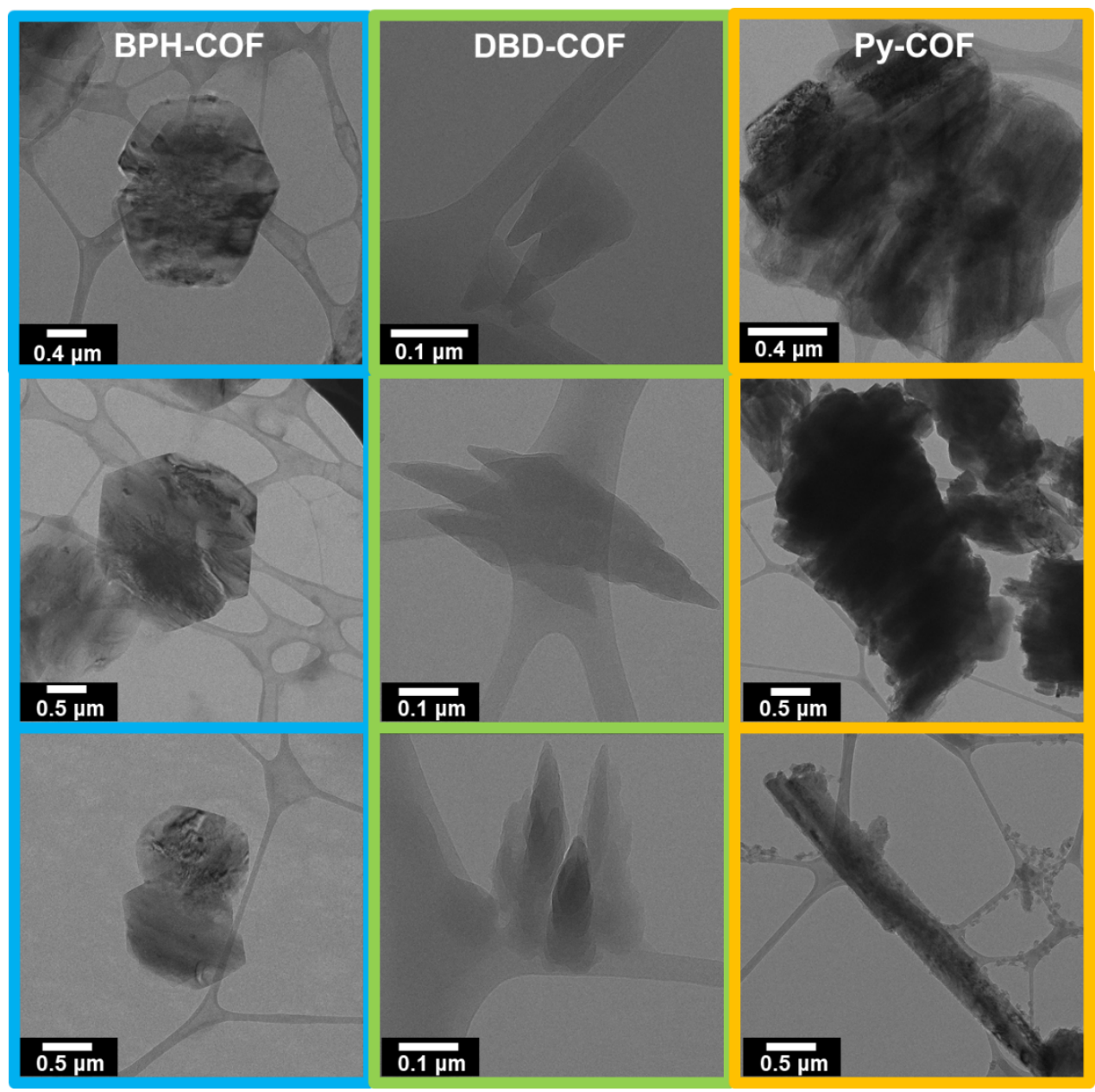

Figure S35. Additional TEM images 


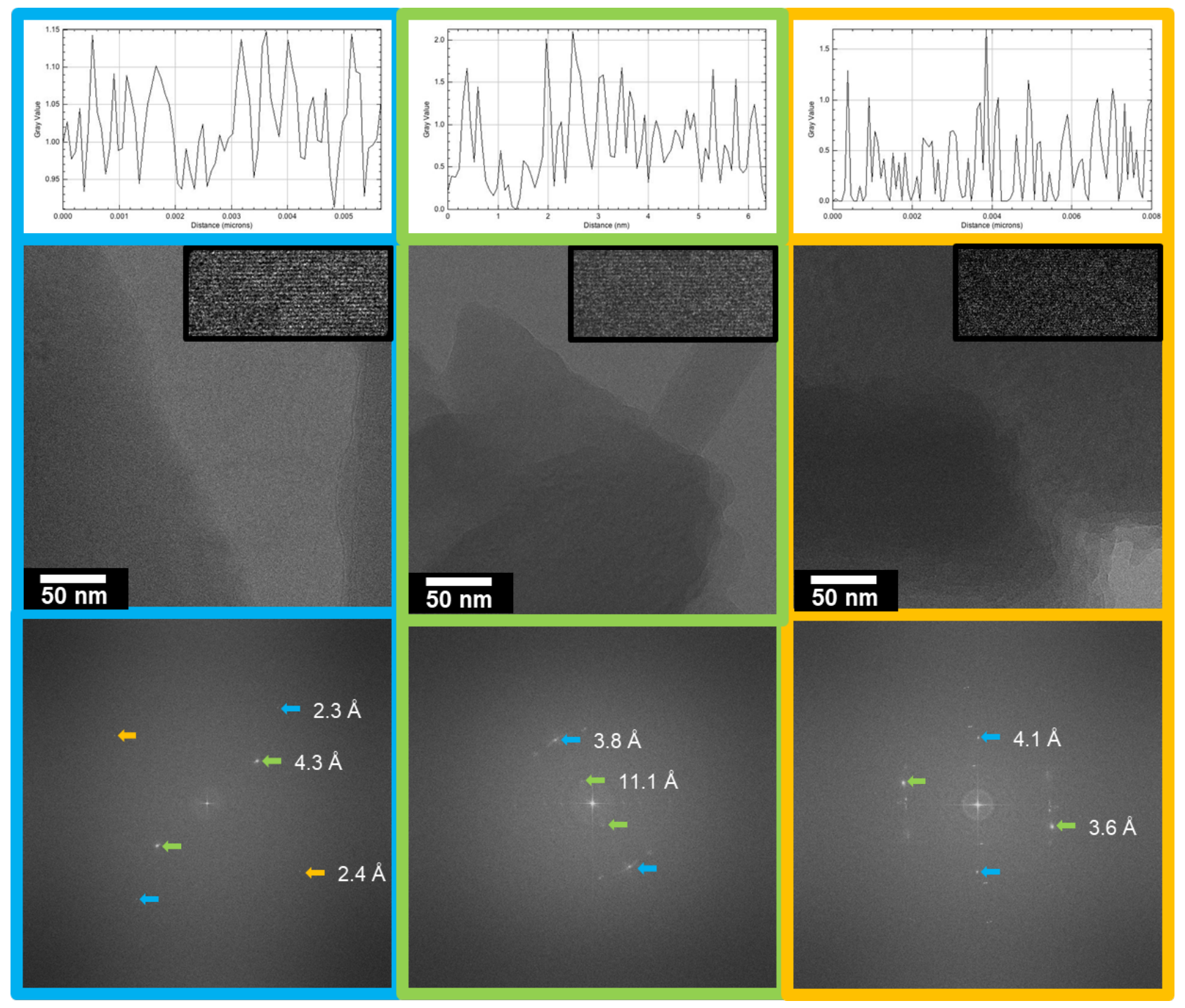

Figure S36. Additional High Resolution TEM images 
XIII. Fluorescence of COF Colloids versus Powders

\section{COF 44}

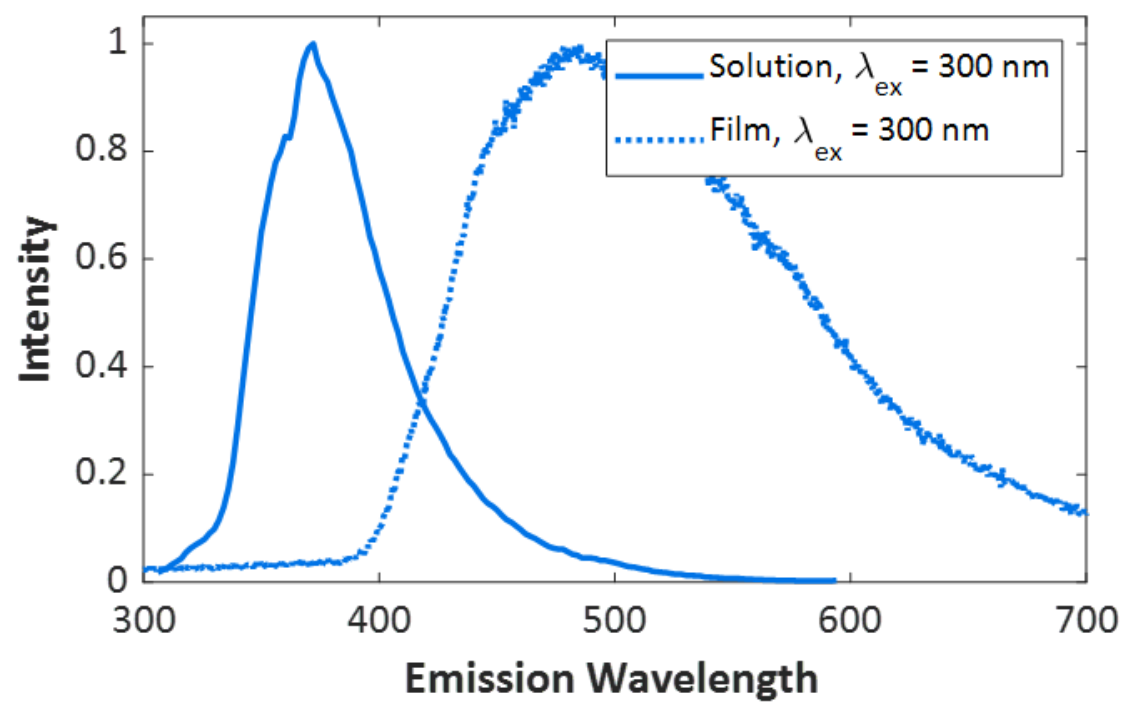

Figure S37. Fluorescence Spectroscopy of Py-COF 


\section{COF 33}

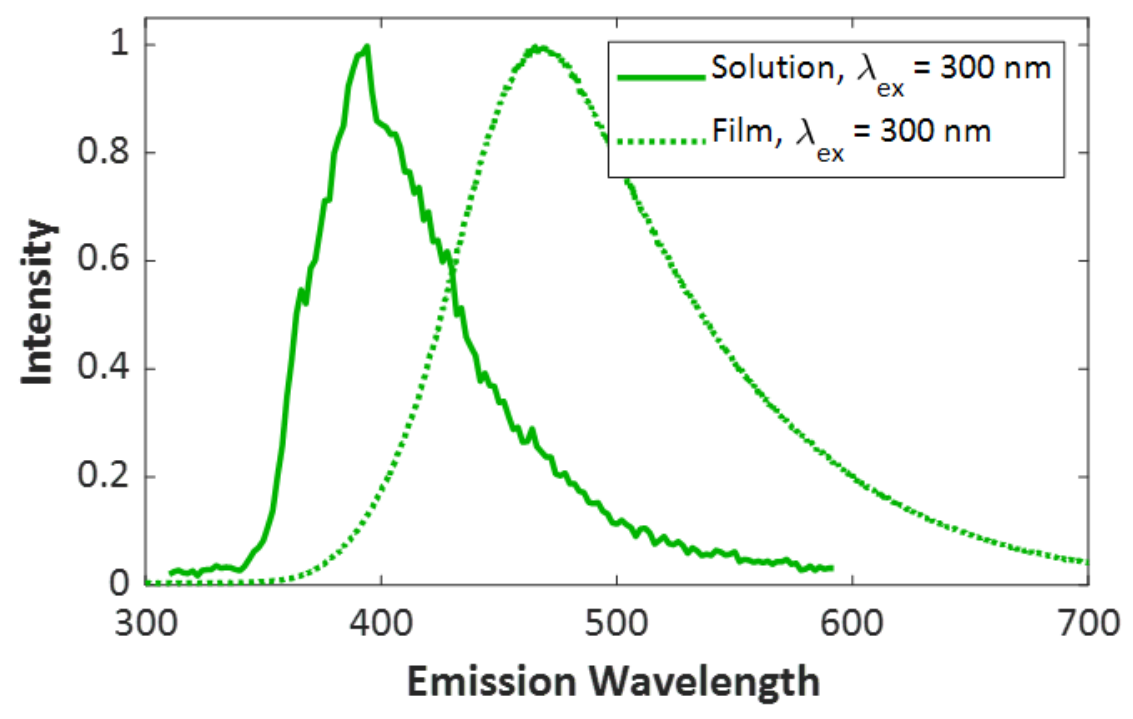

Figure S38. Fluorescence Spectroscopy of DBD-COF 


\section{References}

1. Jiang, Z., GIXSGUI: a MATLAB toolbox for grazing-incidence X-ray scattering data visualization and reduction, and indexing of buried three-dimensional periodic nanostructured films. $J$. Appl. Cryst. 2015, 48 (3), 917-926.

2. Studio, D., Accelrys Inc. San Diego, CA, USA 2013.

3. Novoselov, K.; Mishchenko, A.; Carvalho, A.; Neto, A. C., 2D materials and van der Waals heterostructures. Science 2016, 353 (6298), aac9439.

4. Schorb, M.; Haberbosch, I.; Hagen, W. J.; Schwab, Y.; Mastronarde, D. N., Software tools for automated transmission electron microscopy. Nat. Methods 2019, 1.

5. Suloway, C.; Pulokas, J.; Fellmann, D.; Cheng, A.; Guerra, F.; Quispe, J.; Stagg, S.; Potter, C. S.; Carragher, B., Automated molecular microscopy: the new Leginon system. J. Struct. Biol. 2005, 151 (1), 41-60.

6. $\quad$ Evans, A. M.; Parent, L. R.; Flanders, N. C.; Bisbey, R. P.; Vitaku, E.; Kirschner, M. S.; Schaller, R. D.; Chen, L. X.; Gianneschi, N. C.; Dichtel, W. R., Seeded growth of single-crystal two-dimensional covalent organic frameworks. Science 2018, 361 (6397), 52-57.

7. $\quad$ Spitler, E. L.; Koo, B. T.; Novotney, J. L.; Colson, J. W.; Uribe-Romo, F. J.; Gutierrez, G. D.; Clancy, P.; Dichtel, W. R., A 2D covalent organic framework with 4.7-nm pores and insight into its interlayer stacking. J. Am. Chem. Soc. 2011, 133 (48), 19416-19421.

8. $\quad \mathrm{Ng}$, M. C.; Harper, J. B.; Stampfl, A. P.; Kearley, G. J.; Rols, S.; Stride, J. A., Central-Atom Size Effects on the Methyl Torsions of Group XIV Tetratolyls. Chem.: Eur. J. 2012, 18 (41), 13018-13024.

9. Fournier, J.-H.; Maris, T.; Wuest, J. D.; Guo, W.; Galoppini, E., Molecular tectonics. Use of the hydrogen bonding of boronic acids to direct supramolecular construction. J. Am. Chem. Soc. 2003, 125

(4), 1002-1006. 


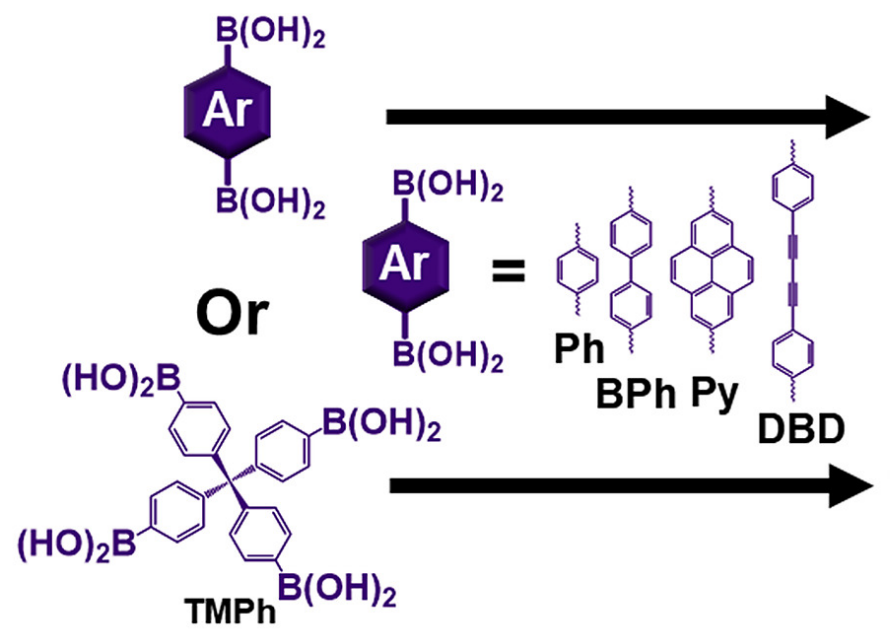

\section{D COFs}
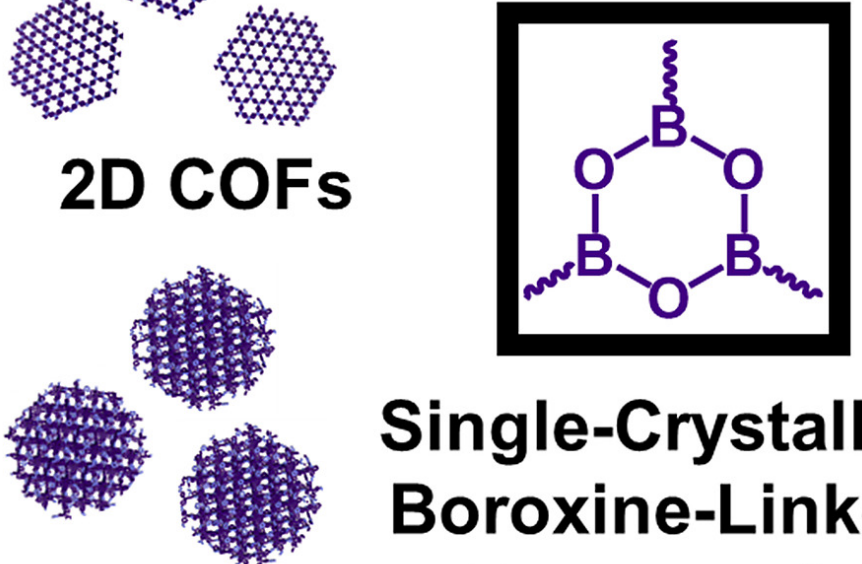

3D COFs

Single-Crystalline Boroxine-Linked Frameworks Boroxine COFs $\sqrt{3 D}$ COFs $\sqrt{\text { Fluorescence Enhancement }}$ 